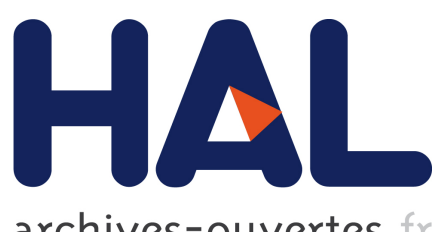

archives-ouvertes

\title{
A second order model for vehicular traffics with local point constraints on the flow
}

B Andreianov, Carlotta Donadello, Massimiliano D. Rosini

\section{To cite this version:}

B Andreianov, Carlotta Donadello, Massimiliano D. Rosini. A second order model for vehicular traffics with local point constraints on the flow. 2015. <hal-01146116>

\section{HAL Id: hal-01146116 \\ https://hal.archives-ouvertes.fr/hal-01146116}

Submitted on 12 May 2015

HAL is a multi-disciplinary open access archive for the deposit and dissemination of scientific research documents, whether they are published or not. The documents may come from teaching and research institutions in France or abroad, or from public or private research centers.
L'archive ouverte pluridisciplinaire HAL, est destinée au dépôt et à la diffusion de documents scientifiques de niveau recherche, publiés ou non, émanant des établissements d'enseignement et de recherche français ou étrangers, des laboratoires publics ou privés. 


\title{
A second order model for vehicular traffics with local point constraints on the flow
}

\author{
B.Andreianov · C.Donadello · M.D.Rosini \\ Received: date / Accepted: date
}

\begin{abstract}
In this paper we present a second order model based on the Aw, Rascle, Zhang model (ARZ) for vehicular traffics subject to point constraints on the flow, its motivation being, for instance, the modeling of traffic lights along a road. We first introduce a definition of entropy solution by choosing a family of entropy pairs analogous to the Kruzhkov entropy pairs for scalar conservation laws; then we apply the wave-front tracking method to prove existence and a priori bounds for the entropy solutions of constrained Cauchy problem for ARZ with initial data of bounded variation and piecewise constant constraints. The case of solutions attaining values at the vacuum is considered. We construct an explicit example to describe some qualitative features of the solutions.
\end{abstract}

Keywords Hyperbolic systems of PDEs with constraints - Wave-front tracking approximations · Traffic modeling $\cdot$ Aw, Rascle, Zhang model

Mathematics Subject Classification (2000) 35L65 - 90B20

Version of April 27, 2015.

\section{Introduction}

This paper deals with macroscopic modeling of traffic flows. Macroscopic models of traffic flows are essential to describe, predict, control and optimize the vehicular traffic along a road or a network of roads, whether highways or urban streets. Several social and economic motivations can be related to the need to minimize traffic congestions and consequently the driving time and the pollution related to traffic. A further motivation is the need to reduce the accident risk in road traffic, a human and social cost that is related not only to the driving behaviour, but also to the management of the roads.

Due to the above motivations, the literature on macroscopic models for traffic flows is already vast and characterized by contributions covering statement of problems, modeling aspects, qualitative analysis, and numerical simulations motivated by their real life applications. Macroscopic models of traffic flows are nowadays a consolidated and nonetheless continuously in ferment field of mathematical research from both theoretical and applied points of view, as the surveys $[7,36,38,43]$ and the books $[27,41]$ demonstrate.

We recall that the independent variables describing vehicular traffics at the macroscopic level are the (mean) density $\rho$, namely the number of vehicles per unit length of the road, the velocity $v$, namely the space covered per unit time by the vehicles, and the flow $f$, namely the number of vehicles per unit time. By definition we have that

$$
f=\rho v
$$

Furthermore, the conservation of the number of vehicles along a road with no entrances or exits is expressed by the scalar Partial Differential Equation (PDE)

$$
\rho_{t}+f_{x}=0
$$

All the authors are supported by French ANR JCJC grant CoToCoLa and Polonium 2014 (French-Polish cooperation program) No.331460NC. The second author is also supported by the Université de Franche-Comté, soutien aux EC 2014. Projekt został sfinansowany ze środków Narodowego Centrum Nauki przyznanych na podstawie decyzji nr: DEC-2011/01/B/ST1/03965.

Boris Andreianov

Laboratoire de Mathématiques, Université de Franche-Comté, 16 route de Gray, 25030 Besançon Cedex, France

E-mail: boris.andreianov@univ-fcomte.fr

Carlotta Donadello

Laboratoire de Mathématiques, Université de Franche-Comté, 16 route de Gray, 25030 Besançon Cedex, France

E-mail: carlotta.donadello@univ-fcomte.fr

Massimiliano Daniele Rosini

ICM, University of Warsaw, ulica Prosta 69, 00-838 Warsaw, Poland

E-mail: mrosini@icm.edu.pl 
To close the system (1.1)-(1.2) of two equations and three unknown variables, a further condition has to be derived. We underline that the only accurate physical law in traffic flow theory is the conservation of the number of vehicles (1.2). Any other assumption results from a coarse approximation of empirical observations. However, as vehicular traffic is influenced by psychological effects, nobody expects that traffic modeling reaches the same accuracy as an exact science (e.g. thermodynamics or Newtonian physics). Nevertheless, macroscopic models help to understand non-trivial properties of vehicular traffics, to predict and optimize them.

First order macroscopic models close the system (1.1)-(1.2) by giving beforehand an explicit expression of one of the three unknown variables in terms of the remaining two. The prototype of the first order models is the Lighthill, Whitham [33] and Richards [39] model (LWR). The basic assumption of LWR is that the velocity of any driver depends on the density alone, namely $v=v(\rho)$. The function $v:\left[0, \rho_{\max }\right] \rightarrow\left[0, v_{\max }\right]$ is given beforehand and is assumed to be $\mathbf{C}^{\mathbf{1}}$, non-increasing with $v(0)=v_{\max }$ and $v\left(\rho_{\max }\right)=0$, where $v_{\max }$ is the maximal speed corresponding to the free road and $\rho_{\max }$ is the maximal density corresponding to the situation in which the vehicles are stuck bumper to bumper. As a result, LWR is given by the scalar conservation law

$$
\rho_{t}+[\rho v(\rho)]_{x}=0
$$

Second order macroscopic models close the system (1.1)-(1.2) by adding a further PDE. The most celebrated second order macroscopic model is the Aw, Rascle [5] and Zhang [44] model (ARZ). Away from the vacuum, ARZ writes

$$
\vec{Y}_{t}+F(\vec{Y})_{x}=\overrightarrow{0}
$$

with

$$
\vec{Y} \doteq(\rho, y)^{T} \in \mathbb{R}_{+}^{2} \backslash\{\overrightarrow{0}\}, \quad F(\vec{Y}) \doteq\left[\frac{y}{\rho}-p(\rho)\right] \vec{Y}
$$

where, in analogy with Euler's equations for fluid dynamics, the quantity $y$ is often called generalized momentum of the vehicles. The "pressure" function $p(\rho) \doteq \rho^{\gamma}, \gamma>0$, plays the role of an anticipation factor, taking into account drivers' reactions to the state of traffic in front of them.

The main drawback of LWR is the unrealistic behaviour of the drivers, who adjust instantaneously their velocities according to the densities they are experiencing (which implies infinite acceleration of the vehicles) and take into account the slightest change in the density. Moreover, experimental data show that the fundamental diagram $(\rho, f)$ is given by a cloud of points rather than being the support of a map $\rho \mapsto[\rho v(\rho)]$. ARZ can be interpreted as a generalization of LWR, possessing a family of fundamental diagram curves, rather than a single one. For this reason ARZ avoids the drawbacks of LWR listed above. Moreover, the empirical tests in [24] show that in many cases ARZ is significantly more accurate than LWR.

In real life applications, the presence along the road of "obstacles", such as construction sites, toll gates, traffic lights, etc., hinders the flow of the vehicles. In these cases, we have to impose the further condition

$$
\rho\left(t, x_{i}\right) v\left(\rho\left(t, x_{i}\right)\right) \leq q_{i}
$$

at each place $x_{i}$ where an obstacle is located, being $q_{i}$ the maximal flow allowed through it. We recall that the concept of point constraints was first introduced in the framework of crowd dynamics in [18] to model the evacuation of a corridor through an exit door. The analytical theory for scalar conservations laws was later stated in [15]. We defer to $[3,4,12,13,16,17,22,23,28,30,40,41]$ for further developments and applications also to vehicular traffics. All these results deal with the scalar case.

The theory of point constraints for general systems of conservation laws is far from being established. A first step in this direction is in the paper [26]. There the authors consider the Riemann problem for ARZ with point constraints. In the present paper we extend this result to general Cauchy problem for ARZ with point constraints. We introduce a definition of entropy solution and prove the corresponding existence results. The key tools are the wave-front tracking method $[15,19]$ and the estimates that permit to exploit wave-front tracking in the BV functional setting. A Temple like functional is proposed in order to compensate, via a kind of potential, the possible increase in variation of Riemann invariants due to interactions at the constraint. Another technical difficulty related to ARZ stems from the fact that system (1.3) degenerates into just one equation at the vacuum, $\rho=0$. In particular, as pointed out in [5], the solutions to (1.3) fail to depend continuously on the initial data in any neighbourhood of $\rho=0$; moreover, as observed in [29], the solutions may experience a sudden increase of the total variation as the vacuum appears. However, a theory of point constraints for (1.3) away from the vacuum is not of practical interest. Indeed, for instance, the vacuum arises in the downstream of a traffic light when it is red. Thus, in the present paper we focus on (1.3) suitably interpreted in the vacuum (we use the Riemann invariants variables of the original system (1.3) and distinguish infinitely many different vacuum states).

The point constraints we can handle here are piecewise constant in time, which are appropriate to model, for instance, the presence of traffic lights or toll gates. This restriction is due to the difficulty of controlling the total variation of approximate solutions, indeed, the Temple-like functional we have found depends in a singular way 
on the constraint level (see Remark 7). Forthcoming studies will be devoted to the situations where the constraint may vary continuously with time, as in $[4,12,13,15,16,17,40]$, and to the cases of non-local constraints, as in [2,3].

The paper is organized as follows. In Section 2 we recall ARZ together with its main properties. More precisely, we first describe in Section 2.1 the Riemann solver $\mathcal{R S}$ introduced in [5] in the coordinates $\vec{W}$; then in Section 2.2 we express ARZ away from the vacuum as a $2 \times 2$ system of conservation laws in the coordinates $\vec{Y}$; finally, in Section 2.3 we introduce the concepts of weak and entropy solutions to the Cauchy problem for ARZ in the coordinates $\vec{W}$, that apply also for solutions attaining values in the vacuum. Section 3 deals with the constrained ARZ in the coordinates $\vec{W}$. More precisely, Section 3.1 deals with the constrained Riemann problems and the constrained Riemann solver $\mathcal{R S}_{q_{0}}$ introduced in [26]; Section 3.2 deals with the definitions of weak and entropy solutions to the constrained Cauchy problem for ARZ and contains Theorem 1, that is the main result of this paper. In Section 4 we apply the model to compute an explicit example reproducing the effects of a traffic light on the traffic along a road. In Section 5 we prove the existence of a constrained entropy solution in the case of a constant constraint. Finally, in Section 6 we prove Theorem 1, namely the existence and a priori bounds for entropy solutions of constrained Cauchy problems for ARZ with initial data of bounded variation and piecewise constant constraints.

\section{ARZ}

In this section we recall ARZ together with its main properties. In order to properly describe ARZ also at the vacuum, we first introduce in Section 2.1 the Riemann solver defined in [5] and here denoted by $\mathcal{R S}$. Introducing ARZ as a Riemann Solver is very convenient as the proof of Theorem 1 is based on the wave-front tracking algorithm, which constructs the approximate solutions by means of the Riemann solver $\mathcal{R} \mathcal{S}$. We choose to introduce $\mathcal{R S}$ in the coordinates $\vec{W}$ given by the Riemann invariants of (1.3) rather than in the coordinates $\vec{Y}$, see $(2.4)$ for the definition of the change of coordinates. The choice of the Riemann invariants as independent variables is in fact convenient to describe the Riemann solver $\mathcal{R} \mathcal{S}$ and ease the forthcoming analysis, as the total variation of the solutions in these coordinates does not increase, see $[25,29,35]$ where this property is exploited to prove existence results for ARZ. Finally, the entropy pairs in the coordinates $\vec{W}$ are well defined, but in the coordinates $\vec{Y}$ they are multi-valued at the vacuum.

In Section 2.2 we study in detail (1.3) away from the vacuum. Then, in Section 2.3 we introduce the concepts of weak and entropy solutions to ARZ that apply also for solutions attaining values in the vacuum.

We recall that the existence result obtained in [25] is valid only away from the vacuum. The existence of solutions attaining also the vacuum state is proved in [29,35], by means of BV bounds on the Riemann invariants or of the compensated compactness method.

\subsection{The Riemann solver $\mathcal{R S}$}

Before describing the Riemann solver $\mathcal{R S}$ associated to ARZ we need to introduce some notations. Consider a road parametrized by the coordinate $x \in \mathbb{R}$, and with vehicles moving in the direction of increasing $x$. Denote by $v(t, x), w(t, x) \in \mathbb{R}_{+}$, respectively, the velocity and the Lagrangian marker of the vehicles at time $t \in \mathbb{R}_{+}$ in $x \in \mathbb{R}$. For any $\vec{W} \doteq(v, w)^{T}$ belonging to $\mathcal{W} \doteq\left\{(v, w)^{T} \in \mathbb{R}_{+}^{2}: v \leq w\right\}$, we define the corresponding density $\rho(\vec{W}) \doteq p^{-1}(w-v) \in \mathbb{R}_{+}$, generalized momentum $y(\vec{W}) \doteq p^{-1}(w-v) w \in \mathbb{R}_{+}$and flow $q(\vec{W}) \doteq p^{-1}(w-v) v \in \mathbb{R}_{+}$. Above, $p \in \mathbf{C}^{\mathbf{0}}\left(\mathbb{R}_{+} ; \mathbb{R}_{+}\right) \cap \mathbf{C}^{2}(] 0,+\infty\left[; \mathbb{R}_{+}\right)$represents the anticipation factor, accounts drivers' reactions to the state of traffic in front of them and satisfies

$$
p(0)=0, \quad p^{\prime}(\rho)>0 \quad \text { and } \quad p^{\prime}(\rho)+\rho p^{\prime \prime}(\rho)>0 \quad \text { for every } \rho>0 .
$$

Beside the above conditions we assume that

$$
\lim _{\rho \downarrow 0}\left[\rho^{2} p^{\prime}(\rho)\right]=0, \quad \quad \lim _{\rho \downarrow 0}\left|\frac{\rho p^{\prime \prime}(\rho)}{p^{\prime}(\rho)}\right|<+\infty .
$$

By definition, we have that the vacuum $\rho=0$ corresponds to the half line $\mathcal{W}_{0} \doteq\left\{(v, w)^{T} \in \mathcal{W}: v=w\right\}$ and the non-vacuum states $\rho \neq 0$ to $\mathcal{W}_{0}^{c} \doteq \mathcal{W} \backslash \mathcal{W}_{0}$. Finally, let $\Pi \in \mathbf{C}^{\mathbf{0}}\left(\mathbb{R}_{+} ; \mathbb{R}_{+}\right) \cap \mathbf{C}^{\mathbf{1}}(] 0,+\infty\left[; \mathbb{R}_{+}\right)$be the inverse function of $\rho \mapsto p(\rho)+\rho p^{\prime}(\rho)$. By $(2.1)$ we have that $\Pi$ is strictly increasing and $\Pi(0)=0$.

We will consider Riemann data in the domain

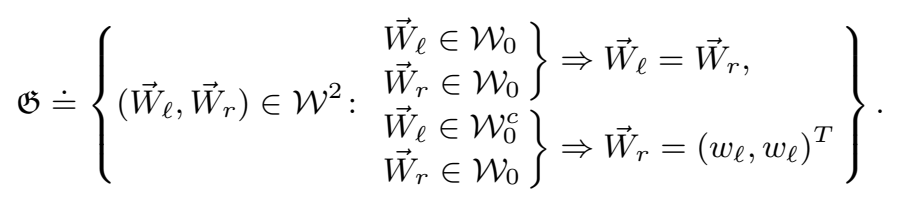


This is justified by the fact that $\mathfrak{G}$ is invariant for the wave-front tracking algorithm introduced below, moreover, every initial datum can be discretized with an approximate datum belonging to $\mathfrak{G}$. Then ARZ corresponds to the Riemann solver

$$
\begin{array}{llll}
\mathcal{R S}: & \rightarrow & \mathbf{C}^{\mathbf{0}}(] 0,+\infty\left[; \mathbf{L}_{\text {loc }}^{\mathbf{l o c}}(\mathbb{R} ; \mathcal{W})\right), \\
& \left(\vec{W}_{\ell}, \vec{W}_{r}\right) & \mapsto & \mathcal{R S}\left[\vec{W}_{\ell}, \vec{W}_{r}\right],
\end{array}
$$

that can be defined by means of the following elementary waves:

- If $\vec{W}_{\ell} \in \mathcal{W}, \vec{W}_{r} \in \mathcal{W}_{0}^{c}$ with $w_{\ell}=w_{r}$ and $v_{r}<v_{\ell}$, then we let

$$
\mathfrak{S}\left[\vec{W}_{\ell}, \vec{W}_{r}\right](x / t) \doteq \begin{cases}\vec{W}_{\ell} & \text { if } x<\sigma\left(\vec{W}_{\ell}, \vec{W}_{r}\right) t, \\ \vec{W}_{r} & \text { if } x>\sigma\left(\vec{W}_{\ell}, \vec{W}_{r}\right) t,\end{cases}
$$

where

$$
\sigma\left(\vec{W}_{\ell}, \vec{W}_{r}\right) \doteq \frac{q\left(\vec{W}_{r}\right)-q\left(\vec{W}_{\ell}\right)}{\rho\left(\vec{W}_{r}\right)-\rho\left(\vec{W}_{\ell}\right)} .
$$

- If $\vec{W}_{\ell} \in \mathcal{W}_{0}^{c}, \vec{W}_{r} \in \mathcal{W}$ with $w_{\ell}=w_{r} \doteq w$ and $v_{\ell}<v_{r}$, then we let

$$
\mathfrak{R}\left[\vec{W}_{\ell}, \vec{W}_{r}\right](x / t) \doteq \begin{cases}\vec{W}_{\ell} & \text { if } x<\lambda_{1}\left(\vec{W}_{\ell}\right) t, \\
\left(\begin{array}{c}
w-p\left(\Pi\left(w-\frac{x}{t}\right)\right) \\
w
\end{array}\right) & \text { if } \lambda_{1}\left(\vec{W}_{\ell}\right) t<x<\lambda_{1}\left(\vec{W}_{r}\right) t \\
\vec{W}_{r} & \text { if } x>\lambda_{1}\left(\vec{W}_{r}\right) t,\end{cases}
$$

where

$$
\lambda_{1}(\vec{W}) \doteq \begin{cases}v-\rho(\vec{W}) p^{\prime}(\rho(\vec{W})) & \text { if } \vec{W} \in \mathcal{W}_{0}^{c} \\ w & \text { if } \vec{W} \in \mathcal{W}_{0}\end{cases}
$$

- If $\vec{W}_{\ell}, \vec{W}_{r} \in \mathcal{W}_{0}^{c}$ with $v_{\ell}=v_{r} \doteq v$ and $w_{\ell} \neq w_{r}$, then we let

$$
\mathfrak{C}\left[\vec{W}_{\ell}, \vec{W}_{r}\right](x / t) \doteq \begin{cases}\vec{W}_{\ell} & \text { if } x<v t \\ \vec{W}_{r} & \text { if } x>v t\end{cases}
$$

Definition 1 (Riemann solver $\mathcal{R S}$ ) For any $\vec{W}_{\ell}=\left(v_{\ell}, w_{\ell}\right)^{T}, \vec{W}_{r}=\left(v_{r}, w_{r}\right)^{T} \in \mathcal{W}$ with $\vec{W}_{\ell} \neq \vec{W}_{r}$ and $\left(\vec{W}_{\ell}, \vec{W}_{r}\right) \in \mathfrak{G}$, we define $\mathcal{R} \mathcal{S}\left[\vec{W}_{\ell}, \vec{W}_{r}\right]$ as follows:

1. If $\vec{W}_{r} \in \mathcal{W}_{0}^{c}, w_{\ell}=w_{r}$ and $v_{r}<v_{\ell}$, then $\mathcal{R S}\left[\vec{W}_{\ell}, \vec{W}_{r}\right] \equiv \mathfrak{S}\left[\vec{W}_{\ell}, \vec{W}_{r}\right]$

2. If $\vec{W}_{\ell} \in \mathcal{W}_{0}^{c}, w_{\ell}=w_{r}$ and $v_{\ell}<v_{r}$, then $\mathcal{R} \mathcal{S}\left[\vec{W}_{\ell}, \vec{W}_{r}\right] \equiv \mathfrak{R}\left[\vec{W}_{\ell}, \vec{W}_{r}\right]$.

3. If $\vec{W}_{\ell}, \vec{W}_{r} \in \mathcal{W}_{0}^{c}$ and $v_{\ell}=v_{r}$, then $\mathcal{R S}\left[\vec{W}_{\ell}, \vec{W}_{r}\right] \equiv \mathfrak{C}\left[\vec{W}_{\ell}, \vec{W}_{r}\right]$.

4. If $\vec{W}_{\ell} \in \mathcal{W}_{0}$ and $\vec{W}_{r} \in \mathcal{W}_{0}^{c}$, then

$$
\mathcal{R S}\left[\vec{W}_{\ell}, \vec{W}_{r}\right](x / t)= \begin{cases}\vec{W}_{\ell} & \text { if } x<v_{r} t \\ \vec{W}_{r} & \text { if } x>v_{r} t\end{cases}
$$

5. If $\vec{W}_{\ell}, \vec{W}_{r} \in \mathcal{W}_{0}^{c}$ and $v_{r}<v_{\ell}<w_{\ell}$, then $\mathcal{R S}\left[\vec{W}_{\ell}, \vec{W}_{r}\right]$ is the juxtaposition of $\mathfrak{S}\left[\vec{W}_{\ell}, \vec{W}_{m}\right]$ and $\mathfrak{C}\left[\vec{W}_{m}, \vec{W}_{r}\right]$, where $\vec{W}_{m}=\left(v_{r}, w_{\ell}\right)^{T} \in \mathcal{W}_{0}^{c}$

6. If $\vec{W}_{\ell}, \vec{W}_{r} \in \mathcal{W}_{0}^{c}$ and $v_{\ell}<v_{r}<w_{\ell}$, then $\mathcal{R} \mathcal{S}\left[\vec{W}_{\ell}, \vec{W}_{r}\right]$ is the juxtaposition of $\mathfrak{R}\left[\vec{W}_{\ell}, \vec{W}_{m}\right]$ and $\mathfrak{C}_{m}\left[\vec{W}_{m}, \vec{W}_{r}\right]$, where $\vec{W}_{m}=\left(v_{r}, w_{\ell}\right)^{T} \in \mathcal{W}_{0}^{c}$.

7. If $\vec{W}_{\ell}, \vec{W}_{r} \in \mathcal{W}_{0}^{c}$ and $v_{\ell}<w_{\ell} \leq v_{r}<w_{r}$, then

$$
\mathcal{R S}\left[\vec{W}_{\ell}, \vec{W}_{r}\right](x / t)= \begin{cases}\mathfrak{R}\left[\vec{W}_{\ell}, \vec{W}_{m}\right](x / t) & \text { if } x<v_{r} t \\ \vec{W}_{r} & \text { if } x>v_{r} t\end{cases}
$$

where $\vec{W}_{m}=\left(w_{\ell}, w_{\ell}\right)^{T} \in \mathcal{W}_{0}$.

Finally, we define $\mathcal{R} \mathcal{S}\left[\vec{W}_{*}, \vec{W}_{*}\right] \equiv \vec{W}_{*}$ for any $\vec{W}_{*} \in \mathcal{W}$.

Let us stress that if $\vec{W}_{\ell} \in \mathcal{W}_{0}^{c}$ and $\vec{W}_{r} \in \mathcal{W}_{0}$, then $\left(\vec{W}_{\ell}, \vec{W}_{r}\right) \in \mathfrak{G}$ if and only if $w_{\ell}=w_{r}$. However, in the case $w_{\ell} \neq w_{r}$ we can define $\mathcal{R} \mathcal{S}\left[\vec{W}_{\ell}, \vec{W}_{r}\right] \equiv \mathfrak{R}\left[\vec{W}_{\ell}, \vec{W}_{m}\right]$, where $\vec{W}_{m}=\left(w_{\ell}, w_{\ell}\right)^{T} \in \mathcal{W}_{0}$. This choice is motivated by the general remark below. 
Remark 1 For any $\vec{W}_{\ell}=\left(v_{*}, w_{*}\right)^{T} \in \mathcal{W}_{0}^{c}$ and $\vec{W}_{r}=\left(v_{*}, v_{*}\right)^{T} \in \mathcal{W}_{0}$, there are in principle two weak solutions for the corresponding Riemann problem. One consisting of the contact discontinuity

$$
\begin{cases}\vec{W}_{\ell} & \text { if } x<v_{*} t \\ \vec{W}_{r} & \text { if } x>v_{*} t\end{cases}
$$

and one which is the juxtaposition of a rarefaction and a discontinuity

$$
\begin{cases}\Re\left[\vec{W}_{\ell}, \vec{W}_{m}\right](x / t) & \text { if } x<w_{*} t \\ \vec{W}_{r} & \text { if } x>w_{*} t\end{cases}
$$

where $\vec{W}_{m}=\left(w_{*}, w_{*}\right)^{T} \in \mathcal{W}_{0}$. As both of these waves do not dissipate the entropy, we cannot use this standard mathematical tool to select one of the two. However, as pointed out in the original paper by Aw and Rascle [5], the rarefaction wave is what we can observe in reality, while the contact discontinuity would contradict the empirical observations. For this reason, we introduced the domain $\mathfrak{G}$ and defined the Riemann solver $\mathcal{R S}$ as above. Then, the selection of physically acceptable solutions is done at the level of approximate solutions constructed with the Riemann solver $\mathcal{R S}$. Let us stress that one could attempt to describe admissibility of general solutions in terms of localized comparison to a given Riemann solver and to prove uniqueness of admissible solutions with tame variation (see $[8,10,11]$ ). However, this is beyond the scope of the present paper.

We conclude the section underlying that from the definition of the Riemann solver $\mathcal{R} \mathcal{S}$, one can verify that the set $\left\{(v, w)^{T} \in \mathcal{W}: v \geq v_{*}\right.$ and $\left.w_{*} \leq w \leq w^{*}\right\}$ is an invariant domain for any $0 \leq v_{*} \leq w_{*}<w^{*}$.

2.2 The system of conservation laws in the coordinates $\vec{Y}$ away from the vacuum

In order to express ARZ as a system of conservation laws we use the coordinates $\vec{Y}$ linked to the coordinates $\vec{W}$ by the following change of coordinates:

$$
Y(\vec{W}) \doteq\left(\begin{array}{c}
\rho(\vec{W}) \\
y(\vec{W})
\end{array}\right) \doteq\left(\begin{array}{c}
p^{-1}(w-v) \\
p^{-1}(w-v) w
\end{array}\right), \quad W(\vec{Y}) \doteq\left(\begin{array}{c}
v(\vec{Y}) \\
w(\vec{Y})
\end{array}\right) \doteq\left(\begin{array}{c}
\frac{y}{\rho}-p(\rho) \\
\frac{y}{\rho}
\end{array}\right) .
$$

By definition $\vec{Y}$ belongs to $\mathcal{D} \doteq\left\{(\rho, y)^{T} \in \mathbb{R}_{+}^{2}: \rho p(\rho) \leq y\right\}$ and the vacuum corresponds to the origin. We stress that $Y$ is one to one with inverse $W$ away from the vacuum. On the other hand, $Y\left(\mathcal{W}_{0}\right)=\{\overrightarrow{0}\}$ and $\vec{Y}$ variables cannot distinguish different states in $\mathcal{W}_{0}$. Observe in particular that if $\vec{W}$ is in $\mathbf{B V}$ and is uniformly away from the vacuum, namely there exists a constant $\varepsilon>0$ such that $\inf _{x \in \mathbb{R}}[w(x)-v(x)] \geq \varepsilon$, then also $Y(\vec{W})$ is in $\mathbf{B V}$.

Away from the vacuum ARZ can be expressed by the system of conservation laws

$$
\vec{Y}_{t}+F(\vec{Y})_{x}=\overrightarrow{0}
$$

with

$$
\vec{Y} \doteq\left(\begin{array}{c}
\rho \\
y
\end{array}\right), \quad F(\vec{Y}) \doteq\left[\frac{y}{\rho}-p(\rho)\right] \vec{Y} .
$$

The eigenvalues of the Jacobian matrix of $F$

$$
A(\vec{Y}) \doteq\left(\begin{array}{cc}
-p(\rho)-\rho p^{\prime}(\rho) & 1 \\
-\frac{y^{2}}{\rho^{2}}-y p^{\prime}(\rho) & 2 \frac{y}{\rho}-p(\rho)
\end{array}\right)
$$

are

$$
\lambda_{1}(\vec{Y}) \doteq \frac{y}{\rho}-p(\rho)-\rho p^{\prime}(\rho), \quad \lambda_{2}(\vec{Y}) \doteq \frac{y}{\rho}-p(\rho),
$$

with corresponding eigenvectors

$$
R_{1}(\vec{Y}) \doteq \vec{Y}, \quad R_{2}(\vec{Y}) \doteq\left(\rho, y+\rho^{2} p^{\prime}(\rho)\right)^{T} .
$$

By a direct computation

$$
\nabla \lambda_{1}(\vec{Y}) \cdot R_{1}(\vec{Y})=-\rho\left[2 p^{\prime}(\rho)+\rho p^{\prime \prime}(\rho)\right], \quad \nabla \lambda_{2}(\vec{Y}) \cdot R_{2}(\vec{Y})=0 .
$$

Hence, away from the vacuum, the system (2.5) is strictly hyperbolic, $\lambda_{1}<\lambda_{2}$, the first characteristic field is genuinely non-linear, $\nabla \lambda_{1} \cdot R_{1}<0$, the second characteristic field is linearly degenerate, $\nabla \lambda_{2} \cdot R_{2} \equiv 0$, and the 
first and second Riemann invariants are respectively $w$ and $v$. Recall that $v$ is the velocity of the vehicles and $w$ is the Lagrangian marker.

Consider waves originating from $(t, x)=(0,0)$. Observe that away from the vacuum the elementary waves introduced in Definition 1 correspond to the elementary waves of system (2.5) under the change of coordinate (2.4). Indeed, the waves of the first family can be shocks or rarefactions, while the waves of the second family are contact discontinuities. More precisely, a wave of the first family from $\vec{Y}_{\ell}$ to $\vec{Y}_{r}$ with $\vec{Y}_{\ell} \neq \vec{Y}_{r}$ and $v\left(\vec{Y}_{\ell}\right) \neq w\left(\vec{Y}_{\ell}\right)=w\left(\vec{Y}_{r}\right) \neq$ $v\left(\vec{Y}_{r}\right)$ is a shock

$$
\begin{cases}\vec{Y}_{\ell} & \text { if } x<\sigma_{Y}\left(\vec{Y}_{\ell}, \vec{Y}_{r}\right) t \\ \vec{Y}_{r} & \text { if } x>\sigma_{Y}\left(\vec{Y}_{\ell}, \vec{Y}_{r}\right) t\end{cases}
$$

with speed of propagation

$$
\sigma_{Y}\left(\vec{Y}_{\ell}, \vec{Y}_{r}\right) \doteq \frac{\rho_{r} v\left(\vec{Y}_{r}\right)-\rho_{\ell} v\left(\vec{Y}_{\ell}\right)}{\rho_{r}-\rho_{\ell}}
$$

if $\rho_{\ell}<\rho_{r}$, otherwise it is a centred rarefaction

$$
\begin{cases}\vec{Y}_{\ell} & \text { if } x<\lambda_{1}\left(\vec{Y}_{\ell}\right) t, \\
\Pi\left(w\left(\vec{Y}_{\ell}\right)-\frac{x}{t}\right)\left(\begin{array}{c}
1 \\
w\left(\vec{Y}_{\ell}\right)
\end{array}\right) & \text { if } \lambda_{1}\left(\vec{Y}_{\ell}\right) t<x<\lambda_{1}\left(\vec{Y}_{r}\right) t, \\
\vec{Y}_{r} & \text { if } x>\lambda_{1}\left(\vec{Y}_{r}\right) t .\end{cases}
$$

We recall that $\Pi$ is the inverse function of $\rho \mapsto p(\rho)+\rho p^{\prime}(\rho)$.

Finally, a wave of the second family from $\vec{Y}_{\ell}$ to $\vec{Y}_{r}$ with $\vec{Y}_{\ell} \neq \vec{Y}_{r}$ and $w\left(\vec{Y}_{\ell}\right) \neq v\left(\vec{Y}_{\ell}\right)=v\left(\vec{Y}_{r}\right) \neq w\left(\vec{Y}_{r}\right)$ is always a contact discontinuity

$$
\begin{cases}\vec{Y}_{\ell} & \text { if } x<v t \\ \vec{Y}_{r} & \text { if } x>v t\end{cases}
$$

with speed of propagation $v=v\left(\vec{Y}_{\ell}\right)=v\left(\vec{Y}_{r}\right)$.

\subsection{Weak and entropy solutions}

In this section we introduce the definition of solutions of the Cauchy problem

$$
Y(\vec{W})_{t}+F(Y(\vec{W}))_{x}=\overrightarrow{0}, \quad \vec{W}(0, x)=\vec{W}_{0}(x),
$$

where the initial datum $\vec{W}_{0}$ is assumed to be in $\mathbf{L}^{\infty}(\mathbb{R} ; \mathcal{W})$. Let $V_{0} \doteq\left\|\vec{W}_{0}\right\|_{\infty}$. A general global existence result for solutions of (2.6) that take values away from the vacuum is not of practical interest. In fact, even if the vacuum is not present initially and does not appear immediately, the solution to (2.6) can reach a vacuum state in finite time, see [29] for an example. An analysis that excludes the vacuum is therefore severely limited. As a first step to cope with solutions involving vacuum states we extend the flux $F$ to the whole $\mathbb{R}_{+}$by taking $F(\overrightarrow{0}) \doteq \overrightarrow{0}$. This choice is motivated by the observation that away from the vacuum $F(\vec{Y})$ is the product between the vector $\vec{Y}$ and the uniformly bounded quantity $[y / \rho-p(\rho)] \in\left[0, V_{0}\right]$.

Definition 2 (Weak solution) Let $\vec{W}_{0} \in \mathbf{L}^{\infty}(\mathbb{R} ; \mathcal{W})$. We say that a function $\vec{W}$ belonging to $\mathbf{L}^{\infty}\left(\mathbb{R}_{+} \times \mathbb{R} ; \mathcal{W}\right) \cap$ $\mathbf{C}^{\mathbf{0}}\left(\mathbb{R}_{+} ; \mathbf{L}_{\text {loc }}^{1}(\mathbb{R} ; \mathcal{W})\right)$ is a weak solution of $(2.6)$ if it satisfies the initial condition $\vec{W}(0, x)=\vec{W}_{0}(x)$ for a.e. $x$ in $\mathbb{R}$ and for any test function $\phi \in \mathbf{C}_{\mathbf{c}}^{\infty}(] 0,+\infty[\times \mathbb{R} ; \mathbb{R})$

$$
\int_{\mathbb{R}_{+}} \int_{\mathbb{R}} \rho(\vec{W})\left[\phi_{t}+v \phi_{x}\right]\left(\begin{array}{c}
1 \\
w
\end{array}\right) \mathrm{d} x \mathrm{~d} t=\overrightarrow{0} .
$$

Proposition 1 For any $\left(\vec{W}_{\ell}, \vec{W}_{r}\right) \in \mathfrak{G}$ we have that $\mathcal{R} \mathcal{S}\left[\vec{W}_{\ell}, \vec{W}_{r}\right]$ is a weak solution of (2.6) with the Riemann datum

$$
\vec{W}_{0}(x)= \begin{cases}\vec{W}_{\ell} & \text { if } x<0 \\ \vec{W}_{r} & \text { if } x>0\end{cases}
$$

Proof Assume that $\mathcal{R S}\left[\vec{W}_{\ell}, \vec{W}_{r}\right]$ performs a discontinuity from $\vec{W}_{\ell}^{*}$ to $\vec{W}_{r}^{*}$ with speed of propagation $\sigma^{*}$. We have to prove that it satisfies the Rankine-Hugoniot jump conditions

$$
\rho\left(\vec{W}_{r}^{*}\right)\left[\sigma^{*}-v_{r}^{*}\right]=\rho\left(\vec{W}_{\ell}^{*}\right)\left[\sigma^{*}-v_{\ell}^{*}\right], \quad \quad \rho\left(\vec{W}_{r}^{*}\right)\left[\sigma^{*}-v_{r}^{*}\right] w_{r}^{*}=\rho\left(\vec{W}_{\ell}^{*}\right)\left[\sigma^{*}-v_{\ell}^{*}\right] w_{\ell}^{*} .
$$

Away from the vacuum the result is obvious. Assume therefore that at least one of the two states $\vec{W}_{\ell}^{*}, \vec{W}_{r}^{*}$ is a vacuum state. Then, the only possibility is that $\vec{W}_{\ell}^{*}$ is a vacuum state but $\vec{W}_{r}^{*}$ not. In this case, the speed of propagation of $\mathcal{R} \mathcal{S}\left[\vec{W}_{\ell}^{*}, \vec{W}_{r}^{*}\right]$ is $v_{r}^{*}$, that satisfies the Rankine-Hugoniot jump conditions. 
It is well known that conservation laws admit in general more than one weak solution, thus raising the need to impose an additional selection criterion, the so called entropy condition [31,34]. Away from the vacuum, as suggested in $[20$, Section 7.4$]$, the entropy pairs $(\mathcal{E}, \mathcal{Q})$ can be computed as functions of the Riemann invariants $(v, w)$ by solving $[20$, Eq. $(7.4 .12)]$

$$
\mathcal{Q}_{w}=v \mathcal{E}_{w},
$$$$
\mathcal{Q}_{v}=\left[v-\rho(\vec{W}) p^{\prime}(\rho(\vec{W}))\right] \mathcal{E}_{v}
$$

and the integrability condition [20, Eq. (7.4.13)]

$$
\mathcal{E}_{w}+\left[1+\frac{\rho(\vec{W}) p^{\prime \prime}(\rho(\vec{W}))}{p^{\prime}(\rho(\vec{W}))}\right] \mathcal{E}_{v}+\rho(\vec{W}) p^{\prime}(\rho(\vec{W})) \mathcal{E}_{w v}=0
$$

The general solutions of the above system are

$$
\begin{aligned}
& \mathcal{E}(\vec{W})=\left[\mathfrak{f}(w)-\int_{0}^{v} \zeta^{\prime}(w-\nu) \mathfrak{g}(\nu) \mathrm{d} \nu\right] p^{-1}(w-v)+\mathfrak{b}, \\
& \mathcal{Q}(\vec{W})=v \mathcal{E}(\vec{W})-\int_{0}^{v} \mathfrak{g}(\nu) \mathrm{d} \nu+\mathfrak{c},
\end{aligned}
$$

where $\mathfrak{f}$ and $\mathfrak{g}$ are arbitrary sufficiently regular functions, $\mathfrak{b}$ and $\mathfrak{c}$ are arbitrary constants and

$$
\zeta(\nu)=\frac{1}{p^{-1}(\nu)}
$$

In the following we will always assume that $\mathfrak{g}^{\prime} \geq 0$, as the entropies satisfying this additional requirement select the shocks of the first family which also appear in the Riemann solver. A series of important remarks is in order.

Remark 2 To justify the above calculations one should require $\mathbf{C}^{2}$ smoothness of $\mathfrak{f}$ and $\mathbf{C}^{1}$ smoothness of $\mathfrak{g}$ but in fact, one can take for $\mathfrak{f}$ any $\mathbf{W}^{2, \infty}$ function and for $\mathfrak{g}$ any Lipschitz continuous function. This readily follows by approximation arguments. In the sequel, we will focus our attention on particular choices of $\mathfrak{g}$ with piecewise constant derivative $\mathfrak{g}^{\prime}$.

Actually, the class of $\mathfrak{f}$ in the above formulas can be considerably extended, as shows the following remark.

Remark 3 Consider any weak solution $\vec{W}$ of (2.6) in the sense of Definition 2 (possibly attaining values in $\mathcal{W}_{0}$ ). Then, by introducing in (2.6) the functions $A(t, x) \doteq \rho(\vec{W}(t, x))$ and $B(t, x) \doteq q(\vec{W}(t, x))$, one sees that the first equation means that the field $(A, B)$ in $(t, x)$ coordinates is divergence-free (in the sense of distributions), while the second equation means that $w$ is a solution (in the sense of distributions) of a continuity equation:

$$
\begin{aligned}
& \operatorname{div}_{(t, x)}(A, B)=0 \\
& {[A w]_{t}+[B w]_{x}=0}
\end{aligned}
$$

Under the assumption (2.9), any smooth solution $w$ of (2.10) satisfies the renormalized identity

$$
[A \mathfrak{f}(w)]_{t}+[B \mathfrak{f}(w)]_{x}=0
$$

for every $\mathbf{C}^{\mathbf{1}}$ function $\mathfrak{f}$. For general weak solutions $w$ of (2.10), proving the renormalization property (2.11) with nonlinear $\mathfrak{f}$ is extremely delicate. We defer to [21] and the references therein for the general case of a multidimensional space. In the case of a one-dimensional space, which is our case, one can apply the specially designed theory of Panov [37]. In [37] it is proved that under the assumption (2.9) and provided $A \geq 0,|B| \leq$ const $|A|$ (which holds true in our case because $0 \leq B \leq V_{0} A$ ), for any weak solution $w$ of (2.10) the renormalized identity (2.11) holds true for every Borel function $\mathfrak{f}$.

Remark 4 The terms in (2.8) involving the function $\mathfrak{f}$ and the constants $\mathfrak{b}, \mathfrak{c}$ do not contribute to the selection of admissible weak solutions. Therefore, they can be set to zero in the considerations of the present section. However, entropies corresponding to a special family of Lipschitz continuous functions $\left(\mathfrak{f}_{k}\right)_{k}$ will play an important role in the definition of solutions to the constrained ARZ studied in the subsequent sections.

Next, the condition $\mathfrak{g}^{\prime} \geq 0$ ensures that entropy dissipates across the shocks introduced by the Riemann solver $\mathcal{R S}$. Moreover, if $\mathfrak{L}$ is the family of Lipschitz functions $\mathfrak{g}:\left[0, V_{0}\right] \rightarrow \mathbb{R}$ such that

$$
\begin{aligned}
& \text { for any } \left.v \in] 0, V_{0}\right] \text { we have } \mathfrak{g}^{\prime}(v) \geq 0 \text { for every } \mathfrak{g} \in \mathfrak{L}, \text { and } \\
& \text { for any } \left.v \in] 0, V_{0}\right] \text { there exists } \mathfrak{g} \in \mathfrak{L} \text { such that } \mathfrak{g}^{\prime}(v)>0,
\end{aligned}
$$

then the entropy criterion

$$
\mathcal{E}^{\mathfrak{g}}(\vec{W})_{t}+\mathcal{Q}^{\mathfrak{g}}(\vec{W})_{x} \leq 0 \text { for every } \mathfrak{g} \in \mathfrak{L}
$$


is satisfied (in the sense of distributions) by those shocks of the first (genuinely nonlinear) family that are introduced by the Riemann solver $\mathcal{R S}$ and it fails for those shocks of the first family that are not used by $\mathcal{R S}$. Here, the superscript $\mathfrak{g}$ over $\mathcal{E}$ and $\mathcal{Q}$ means that $\mathfrak{g}$ in (2.8) is fixed (while $\mathfrak{f}, \mathfrak{b}$ and $\mathfrak{c}$ can be arbitrary, but one can fix them to be zero for the sake of simplicity).

This claim is easily deduced by considering the family of entropies corresponding to the choice $\left\{\mathfrak{g}_{k}(v)=\right.$ $\left.\operatorname{sign}^{+}(v-k): k \in\right] 0,+\infty[\}$ made below. Indeed, this family plays the role analogous to Kruzhkov entropies for the scalar case: any arbitrary function $\mathfrak{g}$ in $\mathfrak{L}$ can be expressed as $\mathfrak{g}(v)=$ const $+\int_{0}^{V_{0}} \mathfrak{g}_{k}(v) \omega(k) \mathrm{d} k$ for some nonnegative weights $\omega$.

It should be noticed that all rarefactions and contact discontinuities of weak solutions satisfy (2.12) with the equality sign. Therefore entropy inequalities cannot be used to determine the admissibility of contact discontinuities. In particular, those contact discontinuities joining vacuum to non-vacuum states that are not used by $\mathcal{R} \mathcal{S}$ (see Remark 1) cannot be prohibited by condition (2.12). To sum up, (2.12) selects as admissible the $\mathcal{R} \mathcal{S}$-solutions, it prohibits "wrong shocks" of the first family, but it cannot prohibit "wrong contact discontinuities" adjacent to vacuum states.

In this paper we will consider the family of the entropy pairs obtained from (2.8) by taking $\mathfrak{f} \equiv 0, \mathfrak{g}_{k}=$ $\operatorname{sign}^{+}(\cdot-k)$ and $\mathfrak{c}=\mathfrak{b}=0$, namely the entropy pairs defined for any fixed $\left.k \in\right] 0,+\infty[$ as

$$
\begin{gathered}
\mathcal{E}_{k}(\vec{W})= \begin{cases}0 & \text { if } v \leq k, \\
1-\frac{p^{-1}(w-v)}{p^{-1}(w-k)} & \text { if } v>k,\end{cases} \\
\mathcal{Q}_{k}(\vec{W})= \begin{cases}0 & \text { if } v \leq k, \\
k-\frac{q(\vec{W})}{p^{-1}(w-k)} & \text { if } v>k .\end{cases}
\end{gathered}
$$

Since the discontinuities may involve vacuum states, we have to prove in full generality the following proposition (in absence of vacuum states, this result is already explained here above).

Proposition 2 For any non-negative test function $\phi \in \mathbf{C}_{\mathbf{c}}^{\infty}(] 0,+\infty[\times \mathbb{R} ; \mathbb{R})$, the entropy dissipation condition

$$
\left.\int_{\mathbb{R}_{+}} \int_{\mathbb{R}}\left[\mathcal{E}_{k}(\vec{W}) \phi_{t}+\mathcal{Q}_{k}(\vec{W}) \phi_{x}\right] \mathrm{d} x \mathrm{~d} t \geq 0, \quad k \in\right] 0,+\infty[
$$

is satisfied by solutions obtained with the Riemann solver $\mathcal{R S}$, and is not satisfied by any self-similar weak solution to the Riemann problem that contains shocks of the first (genuinely nonlinear) family not present in $\mathcal{R} \mathcal{S}$-solutions.

Proof Let us first observe that $\mathcal{R S}$-solutions satisfy the entropy inequalities if and only if each of the performed discontinuities satisfies the associated dissipation condition (possibly with equality, as it is the case for contact discontinuities). Consider a discontinuity from $\vec{W}_{\ell}$ to $\vec{W}_{r}$ with speed of propagation $\dot{s}$. We have to prove that

$$
\star=\dot{s}\left[\mathcal{E}_{k}\left(\vec{W}_{r}\right)-\mathcal{E}_{k}\left(\vec{W}_{\ell}\right)\right]-\left[\mathcal{Q}_{k}\left(\vec{W}_{r}\right)-\mathcal{Q}_{k}\left(\vec{W}_{\ell}\right)\right] \geq 0
$$

For notational simplicity, let $\rho_{*} \doteq \rho\left(\vec{W}_{*}\right), q_{*} \doteq q\left(\vec{W}_{*}\right), \sigma \doteq \sigma\left(\vec{W}_{\ell}, \vec{W}_{r}\right)$ and $h \doteq p^{-1}(w-k)$. If the discontinuity performed by the $\mathcal{R} \mathcal{S}$-solution is a shock, then $w_{\ell}=w_{r} \doteq w, v_{\ell}>v_{r}$ and $\dot{s}=\sigma$. If $k \in\left[v_{r}, v_{\ell}[\right.$, namely $\left.h \in] \rho_{\ell}, \rho_{r}\right]$, then by (2.1) we have

$$
h p(h) \leq \rho_{\ell} p\left(\rho_{\ell}\right)+\frac{\rho_{\ell} p\left(\rho_{\ell}\right)-\rho_{r} p\left(\rho_{r}\right)}{\rho_{\ell}-\rho_{r}}\left[h-\rho_{\ell}\right]=\rho_{\ell} p\left(\rho_{\ell}\right)+[w-\sigma]\left[h-\rho_{\ell}\right]=\left[\sigma-v_{\ell}\right] \rho_{\ell}+[w-\sigma] h,
$$

and therefore

$$
\begin{aligned}
\star & =\sigma\left[\mathcal{E}_{k}\left(\vec{W}_{r}\right)-\mathcal{E}_{k}\left(\vec{W}_{\ell}\right)\right]-\left[\mathcal{Q}_{k}\left(\vec{W}_{r}\right)-\mathcal{Q}_{k}\left(\vec{W}_{\ell}\right)\right]=\frac{q_{r}-q_{\ell}}{\rho_{r}-\rho_{\ell}}\left[\frac{\rho_{\ell}}{h}-1\right]+k-\frac{q_{\ell}}{h} \\
& =\left[\frac{q_{r}-q_{\ell}}{\rho_{r}-\rho_{\ell}}-v_{\ell}\right] \frac{\rho_{\ell}}{h}-\frac{q_{r}-q_{\ell}}{\rho_{r}-\rho_{\ell}}+k=\left[\sigma-v_{\ell}\right] \frac{\rho_{\ell}}{h}-\sigma+w-p(h) \geq 0 .
\end{aligned}
$$

Moreover, for shocks of the first family not used by $\mathcal{R S}$, we have $v_{\ell}<v_{r}$ and the same calculation shows that the entropy is anti-dissipated. Therefore, these shocks are prohibited. If $k \in] 0,+\infty\left[\backslash\left[v_{r}, v_{\ell}[\right.\right.$, then it is easy to prove that $\star=0$. Finally, if the discontinuity is a contact discontinuity, then $v_{\ell}=v_{r}=\dot{s}$ and $w_{\ell} \neq w_{r}$. Direct computations show that in this case $\star=0$.

Definition 3 (Entropy solution) Let $\vec{W}_{0} \in \mathbf{L}^{\infty}(\mathbb{R} ; \mathcal{W})$. We say that a weak solution $\vec{W}$ in $\mathbf{L}^{\infty}(\mathbb{R}+\times \mathbb{R} ; \mathcal{W}) \cap$ $\mathbf{C}^{\mathbf{0}}\left(\mathbb{R}_{+} ; \mathbf{L}_{\text {loc }}^{\mathbf{1}}(\mathbb{R} ; \mathcal{W})\right)$ of $(2.6)$ in the sense of Definition 2 is an entropy solution if (2.14) holds for any non-negative test function $\phi \in \mathbf{C}_{\mathbf{c}}^{\infty}(] 0,+\infty[\times \mathbb{R} ; \mathbb{R})$ and for any entropy pair $(2.13)$ with $\left.k \in\right] 0,+\infty[$. 
Condition (2.14) originates from the classical definition of entropy solutions to hyperbolic systems of conservation laws introduced by Lax [31] and Liu [34]. However our approach is not the usual one, as instead of determining the adequate Riemann solver by asking that convex entropies of the system are dissipated, we proceed by postulating the Riemann solver and then we prove its entropy dissipation properties. As a result, the condition of convexity of the entropies $\mathfrak{f}$ and $\mathcal{E}_{k}$ is not imposed; this is related to the fact that, first, the dissipation of entropies $\mathfrak{f}$ is zero irrespective of their shape; and second, there is no "natural" form of parabolic regularization for ARZ (the convexity of entropies being linked to particular choice of parabolic "vanishing viscosity" regularization). However, observe in passing that the entropies $\mathcal{E}_{k}$ are $\rho$-convex, see [29].

Even for BV entropy solutions not containing vacuum, we are not able to prove uniqueness for the Cauchy problem (2.6) without enforcing a restrictive assumption on the smallness of the BV norm of the data, see [9]. However, as pointed out in Remark 1, at the vacuum the entropy conditions cannot ensure the uniqueness or that the solution is physically reasonable. A possible criterion (at vacuum) for selection of admissible entropy solutions with bounded variation is the condition

$$
\left(\vec{W}\left(t, x^{-}\right), \vec{W}\left(t, x^{+}\right)\right) \in \mathfrak{G}, \quad t \in \mathbb{R}_{+}, x \in \mathbb{R},
$$

where $\vec{W}\left(t, x^{-}\right)$and $\vec{W}\left(t, x^{+}\right)$denote the traces of the $\mathbf{B V}$ solution $\vec{W}$ on a Lipschitz curve of jump (see [42] for precise formulation of the regularity of $\mathbf{B V}$ functions) and $\mathfrak{G}$ is defined in (2.3). It is easy to show that the condition (2.15) is preserved during the time evolution of the approximate solutions $\vec{W}^{h}$ (constructed in Section 5), provided this condition is satisfied by the approximate initial data $\vec{W}_{0}^{h}$. More precisely, we have

Lemma 1 Let $\vec{W}_{0}^{h} \in \mathbf{L}^{\infty}(\mathbb{R} ; \mathcal{W})$ and let $\vec{W}^{h}$ be the approximate solution constructed in Section 5 . If $\vec{W}_{0}^{h}$ satisfies the property (2.15), then also $\vec{W}^{h}(t), t>0$, satisfies it.

Proof The approximate solution $\vec{W}^{h}$ is constructed by applying the wave-front tracking method and the approximate Riemann solver $\mathcal{R} \mathcal{S}^{h}$, obtained from $\mathcal{R S}$ by discretizing the rarefactions with some step $\varepsilon^{h}$, see Section 5.2 . To prove (2.15) it is sufficient to observe that for any $\vec{W}_{\ell}^{h}, \vec{W}_{r}^{h} \in \mathcal{W}^{h} \cap \mathcal{W}_{0}$ and $\vec{W}_{m}^{h} \in \mathcal{W}^{h} \backslash \mathcal{W}_{0}$ such that $\left(\vec{W}_{\ell}^{h}, \vec{W}_{m}^{h}\right),\left(\vec{W}_{m}^{h}, \vec{W}_{r}^{h}\right)$ belong to $\mathfrak{G}$, the discontinuity $\mathcal{R S}^{h}\left[\vec{W}_{\ell}^{h}, \vec{W}_{m}^{h}\right]$ has speed of propagation $v_{m}^{h}$, the rightmost wave of the discretized rarefaction $\mathcal{R S}^{h}\left[\vec{W}_{m}^{h}, \vec{W}_{r}^{h}\right]$ has speed of propagation $\left(w_{m}^{h}-\varepsilon^{h}\right)$ and that, by assumption, $v_{m}^{h} \leq w_{m}^{h}-\varepsilon^{h}$. Therefore, despite the fact that the leftmost wave accelerates while it interacts with the discretized rarefaction, it cannot reach the rightmost wave.

Observe that given BV initial data in coordinates $\vec{Y}$, one can always define approximate initial data $\vec{W}_{0}^{h}$ that satisfy (2.15). Thus for BV initial data, we are able to construct approximate solutions $\vec{W}^{h}$ (converging pointwise to an entropy solution $\vec{W}$ of (2.6) in the sense of Definition 3) that verify this criterion. However, we are not able to prove that this property is inherited by the entropy solution $\vec{W}$ obtained as the limit of the approximation scheme: This is due to the fact that the limit solution $\vec{W}$ might contain states that belong to $\mathcal{W}_{0}$ but that appear as the limit of states in $\vec{W}^{h}$ that belong to $\mathcal{W}_{0}^{c}$. We think that, in practice, this difficulty can be avoided if one chooses to discretize $\vec{W}_{0}$ by using vacuum states whenever the initial density $\rho_{0}$ is below some threshold level $\rho_{\text {min }}^{h}$, with $\rho_{\min }^{h} \rightarrow 0$ as $h$ goes to infinity. However, precise results in this direction are beyond the scope of the present work.

\section{Constrained ARZ}

In this section we introduce a rigorous definition of solution to ARZ with a point constraint. More precisely, in Section 3.1 we consider the Riemann problem with constant constraint [26], while in Section 3.2 we state the main result of this paper, namely existence and a priori bounds for solutions to the general Cauchy problem subject to a piecewise constant time-dependent point constraint, see Theorem 1 (the proof of Theorem 1 is contained in Sections 5 and 6$)$.

\subsection{The constrained Riemann problem}

Assume that the presence of a toll gate (or a construction site, etc.) hinders the flow at $x=0$. Then, together with the Cauchy problem (2.6) we have to impose the constraint condition

$$
q\left(\vec{W}\left(t, 0^{ \pm}\right)\right) \leq q_{0}
$$

where the constant $q_{0} \geq 0$ prescribes the maximal flow allowed at $x=0$. Above, $\vec{W}\left(t, 0^{-}\right)$denotes the left measure theoretic trace along $x=0$, implicitly defined by

$$
\lim _{\varepsilon \downarrow 0} \frac{1}{\varepsilon} \int_{\mathbb{R}_{+}} \int_{-\varepsilon}^{0}\left\|\vec{W}(t, x)-\vec{W}\left(t, 0^{-}\right)\right\| \phi(t, x) \mathrm{d} x \mathrm{~d} t=0
$$


for all $\phi \in \mathbf{C}_{\mathbf{c}}^{\infty}\left(\mathbb{R}^{2} ; \mathbb{R}\right)$. The right measure theoretic trace, $\vec{W}\left(t, 0^{+}\right)$, is defined analogously. Roughly speaking, the existence of the measure theoretic traces for weak solutions is guaranteed in presence of appropriate $\mathbf{B V}$ bounds, which is our setting in this paper; see [20, Lemma 1.3.3] for the precise statement.

Notice that it is possible to give a sense to inequality (3.1) even for weak traces, see [14]; we briefly address this generalization in Remark 5 below.

In general, the solutions constructed with the Riemann solver $\mathcal{R S}$ do not satisfy (3.1). For this reason, a new Riemann solver has to be introduced. A Riemann solver that always gives solutions satisfying (3.1) is said to be a constrained Riemann solver. In [26] the authors proposed two distinct constrained Riemann solvers. Since only one of them is conservative, Definition 3 naturally selects that one, denoted in [26] by $\mathcal{R} \mathcal{S}_{1}^{q}$ and denoted in this paper by $\mathcal{R S}_{q_{0}}$. For completeness, in this section we recall $\mathcal{R} \mathcal{S}_{q_{0}}$ together with its main properties.

We first introduce some notations, see Figure 1. For any fixed $w>0$, introduce $\dot{W}(w) \doteq(\dot{v}(w), w)^{T} \in \mathcal{W}$ with $\dot{v}(w) \doteq w-p(\dot{\rho}(w))$, where $\dot{\rho}(w)$ corresponds to the maximum of $\rho \mapsto[w-p(\rho)] \rho$ and is implicitly defined by

$$
w=p(\dot{\rho}(w))+\dot{\rho}(w) p^{\prime}(\dot{\rho}(w)) .
$$

Let $\dot{q}(w) \doteq \max \{q(v, w): 0 \leq v \leq w\}$, and observe that by definition $\dot{q}(w)=q(\dot{W}(w))$. Introduce $\tilde{W}\left(q_{0}\right) \doteq$ $\left(\tilde{v}\left(q_{0}\right), \tilde{w}\left(q_{0}\right)\right)^{T} \in \mathcal{W}$ corresponding to the point of the curve $q(\vec{W})=q_{0}$ with the lowest coordinate $w$ and implicitly defined by

$$
\frac{\tilde{v}\left(q_{0}\right)^{2}}{q_{0}}=p^{\prime}\left(\frac{q_{0}}{\tilde{v}\left(q_{0}\right)}\right), \quad \tilde{w}\left(q_{0}\right)=\tilde{v}\left(q_{0}\right)+p\left(\frac{q_{0}}{\tilde{v}\left(q_{0}\right)}\right) .
$$

If $w \geq \tilde{w}\left(q_{0}\right)$, then $\{v \in] 0, w\left[: w=v+p\left(q_{0} / v\right)\right\}$ is not empty and we can define

$$
\hat{W}\left(q_{0}, w\right) \doteq\left(\hat{v}\left(q_{0}, w\right), w\right)^{T}, \quad \quad \check{W}\left(q_{0}, w\right) \doteq\left(\check{v}\left(q_{0}, w\right), w\right)^{T}
$$

by taking

$$
\begin{aligned}
& \hat{v}\left(q_{0}, w\right) \doteq \begin{cases}\min \{v \in] 0, w\left[: w=v+p\left(\frac{q_{0}}{v}\right)\right\} & \text { if } w \in\left[\tilde{w}\left(q_{0}\right),+\infty[,\right. \\
\dot{v}(w) & \text { if } w \in\left[0, \tilde{w}\left(q_{0}\right)[,\right.\end{cases} \\
& \check{v}\left(q_{0}, w\right) \doteq \begin{cases}\max \{v \in] 0, w\left[: w=v+p\left(\frac{q_{0}}{v}\right)\right\} & \text { if } w \in\left[\tilde{w}\left(q_{0}\right),+\infty[,\right. \\
\dot{v}(w) & \text { if } w \in\left[0, \tilde{w}\left(q_{0}\right)[.\right.\end{cases}
\end{aligned}
$$
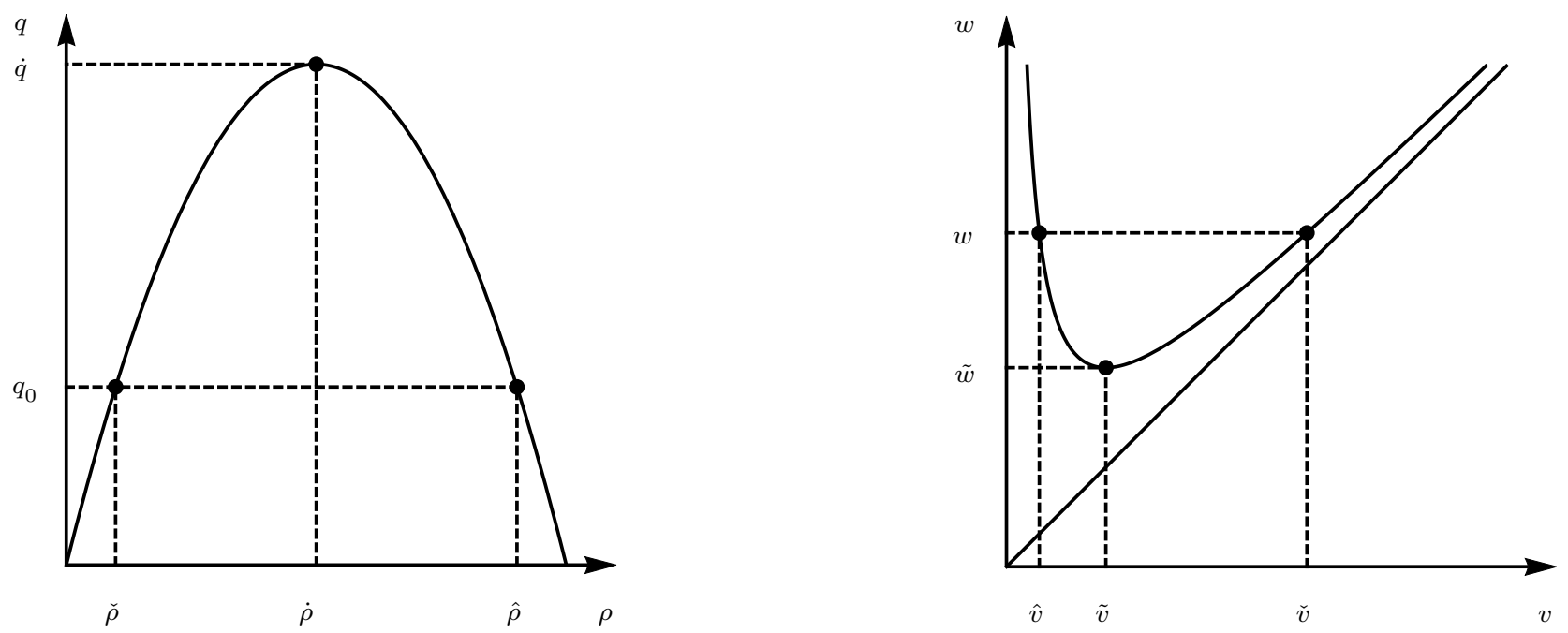

Fig. 1 Above $\hat{\rho} \doteq q_{0} / \hat{v}(w), \check{\rho} \doteq q_{0} / \check{v}(w), \dot{\rho}=\dot{\rho}(w), \hat{v}=\hat{v}(w), \check{v}=\check{v}(w), \dot{v}=\dot{v}(w)$ and $\dot{q}=\dot{q}(w)$. The curve on the left, respectively on the right, is the support of $\rho \mapsto[w-p(\rho)] \rho$ in the $(\rho, q)$-plane, respectively of $v \mapsto v+p\left(q_{0} / v\right)$ in the $(v, w)$-plane.

We can now define the constrained Riemann solver

$$
\begin{array}{llll}
\mathcal{R S}_{q_{0}} & : & \rightarrow & \mathbf{C}^{\mathbf{0}}\left(\mathbb{R}_{+} ; \mathbf{L}_{\text {loc }}^{1}(\mathbb{R} ; \mathcal{W})\right), \\
& \left(\vec{W}_{\ell}, \vec{W}_{r}\right) & \mapsto & \mathcal{R} \mathcal{S}_{q_{0}}\left[\vec{W}_{\ell}, \vec{W}_{r}\right] .
\end{array}
$$


Definition 4 (The constrained Riemann solver $\mathcal{R S}_{q_{0}}$ ) The constrained Riemann solver $\mathcal{R S}_{q_{0}}$ computed at $\left(\vec{W}_{\ell}, \vec{W}_{r}\right) \in \mathfrak{G}$ is defined as follows:

- if $\mathcal{R} \mathcal{S}\left[\vec{W}_{\ell}, \vec{W}_{r}\right]$ satisfies $(3.1)$, then

$$
\mathcal{R} \mathcal{S}_{q_{0}}\left[\vec{W}_{\ell}, \vec{W}_{r}\right] \equiv \mathcal{R S}\left[\vec{W}_{\ell}, \vec{W}_{r}\right]
$$

- otherwise we let

$$
\mathcal{R S}_{q_{0}}\left[\vec{W}_{\ell}, \vec{W}_{r}\right](x / t)= \begin{cases}\mathcal{R} \mathcal{S}\left[\vec{W}_{\ell}, \hat{W}\left(q_{0}, w_{\ell}\right)\right](x / t) & \text { if } x<0 \\ \mathcal{R} \mathcal{S}\left[\check{W}_{\left.\left(q_{0}, w_{\ell}\right), \vec{W}_{r}\right](x / t)}\right. & \text { if } x>0\end{cases}
$$

Observe that if $\vec{W}_{\ell} \in \mathcal{W}_{0}$, then $\mathcal{R S}\left[\vec{W}_{\ell}, \vec{W}_{r}\right]\left(0^{ \pm}\right)=\vec{W}_{\ell}$ satisfies the constraint condition (3.1) and $\mathcal{R} \mathcal{S}_{q_{0}}\left[\vec{W}_{\ell}, \vec{W}_{r}\right] \equiv$ $\mathcal{R S}\left[\vec{W}_{\ell}, \vec{W}_{r}\right]$.

From the definition of $\mathcal{R S}_{q_{0}}$, one can verify that $\left\{(v, w)^{T} \in \mathcal{W}: v \geq v_{*}\right.$ and $\left.w_{*} \leq w \leq w^{*}\right\}$ is an invariant domain for any $0 \leq v_{*} \leq w_{*}<w^{*}$ that satisfy one of the following conditions

$$
\begin{array}{lll}
\text { (i) } w^{*} \leq \tilde{w}, & \text { (ii) } w^{*}>\tilde{w} \text { and } v_{*} \geq \check{v}\left(w^{*}\right), \quad \text { (iii) } w^{*}>\tilde{w} \text { and } v_{*} \leq \hat{v}\left(w^{*}\right) .
\end{array}
$$

For later use we introduce the function $J$ defined as follows

$$
\left.\left(q_{0}, w\right) \in\right] 0,+\infty\left[\times \mathbb{R}_{+} \mapsto J\left(q_{0}, w\right) \doteq \check{v}\left(q_{0}, w\right)-\hat{v}\left(q_{0}, w\right) \in \mathbb{R}_{+}\right.
$$

and prove the following result

Lemma 2 For any $w>\tilde{w}\left(q_{0}\right)$ we have

1. $\partial_{w} \check{v}\left(q_{0}, w\right)>1, \partial_{w}^{2} \check{v}\left(q_{0}, w\right)<0$ and $\lim _{w \downarrow \tilde{w}} \partial_{w} \check{v}\left(q_{0}, w\right)=+\infty$;

2. $\partial_{w} \hat{v}\left(q_{0}, w\right)<0, \partial_{w}^{2} \hat{v}\left(q_{0}, w\right)>0$ and $\lim _{w \downarrow \tilde{w}} \partial_{w} \hat{v}\left(q_{0}, w\right)=-\infty$;

3. $\partial_{w} J\left(q_{0}, w\right)>1, \partial_{w}^{2} J\left(q_{0}, w\right)<0$ and $\lim _{w \downarrow \tilde{w}} \partial_{w} J\left(q_{0}, w\right)=+\infty$.

Proof Let $w>\tilde{w}\left(q_{0}\right)$. Since $[(w-p(\rho)) \rho]_{\rho}=w-p(\rho)-\rho p^{\prime}(\rho)$, by definition we have

$$
w-p\left(\frac{q_{0}}{\check{v}\left(q_{0}, w\right)}\right)-\frac{q_{0}}{\check{v}\left(q_{0}, w\right)} p^{\prime}\left(\frac{q_{0}}{\check{v}\left(q_{0}, w\right)}\right)>0, \quad w=\check{v}\left(q_{0}, w\right)+p\left(\frac{q_{0}}{\check{v}\left(q_{0}, w\right)}\right),
$$

and therefore

$$
\frac{q_{0}}{\check{v}\left(q_{0}, w\right)^{2}} p^{\prime}\left(\frac{q_{0}}{\check{v}\left(q_{0}, w\right)}\right)<1, \quad \partial_{w} \check{v}\left(q_{0}, w\right)=\left[1-\frac{q_{0}}{\check{v}\left(q_{0}, w\right)^{2}} p^{\prime}\left(\frac{q_{0}}{\check{v}\left(q_{0}, w\right)}\right)\right]^{-1} .
$$

Hence $\partial_{w} \check{v}\left(q_{0}, w\right)>0$. Moreover

$$
\partial_{w}^{2} \check{v}\left(q_{0}, w\right)=-q_{0}\left[\frac{\partial_{w} \check{v}\left(q_{0}, w\right)}{\check{v}\left(q_{0}, w\right)}\right]^{3}\left[2 p^{\prime}\left(\frac{q_{0}}{\check{v}\left(q_{0}, w\right)}\right)+\frac{q_{0}}{\check{v}\left(q_{0}, w\right)} p^{\prime \prime}\left(\frac{q_{0}}{\check{v}\left(q_{0}, w\right)}\right)\right]<0 .
$$

Finally, to conclude the proof of the first point it is sufficient to observe that $\lim _{w \rightarrow+\infty} \check{v}(w)=+\infty$ and to use condition (2.2). The proof of the second point is analogous, while the last point follows from the previous ones.

\subsection{The constrained Cauchy problem}

In this section we begin the study of the constrained Cauchy problem

$$
\begin{array}{ll}
Y(\vec{W})_{t}+F(Y(\vec{W}))_{x}=0, & t>0, x \in \mathbb{R}, \\
\vec{W}(0, x)=\vec{W}_{0}(x), & x \in \mathbb{R}, \\
q\left(\vec{W}\left(t, 0^{ \pm}\right)\right) \leq q_{0}(t), & t>0 .
\end{array}
$$

Above, $\vec{W}_{0}$ is assumed to be in $\mathbf{L}^{\infty}(\mathbb{R} ; \mathcal{W})$ with $V_{0} \doteq\left\|\vec{W}_{0}\right\|_{\infty}$ and $q_{0}: \mathbb{R}_{+} \rightarrow \mathbb{R}_{+}$prescribes the maximal flow allowed at $x=0$. The expression for $q_{0}$ depends on the situation we aim to represent. In Section 4 we provide a basic example.

As a first step, we adapt the definitions of weak and entropy solutions, respectively given in Definition 2 and Definition 3 to the problem (3.4).

Definition 5 (Constrained weak solution) Let $\vec{W}_{0} \in \mathbf{L}^{\infty}(\mathbb{R} ; \mathcal{W})$. We say that a function $\vec{W}$ belonging to $\mathbf{L}^{\infty}\left(\mathbb{R}_{+} \times \mathbb{R} ; \mathcal{W}\right) \cap \mathbf{C}^{\mathbf{0}}\left(\mathbb{R}_{+} ; \mathbf{L}_{\text {loc }}^{\mathbf{1}}(\mathbb{R} ; \mathcal{W})\right)$ is a constrained weak solution of $(3.4)$ if

1. $\vec{W}$ is a weak solution of $(3.4 \mathrm{a}),(3.4 \mathrm{~b})$ in the sense of Definition 2; 
2. $\vec{W}$ satisfies the constraint (3.4c), namely

$$
q\left(\vec{W}\left(t, 0^{ \pm}\right)\right) \leq q_{0}(t) \quad \text { for a.e. } t>0
$$

For the definition of constrained entropy solution, let us limit our attention to $\mathbf{L}^{\infty}(] 0,+\infty[; \mathbf{B V}(\mathbb{R} ; \mathcal{W}))$ functions $\vec{W}$. This is enough for our purposes because the $\mathbf{B V}$ bounds in space are an essential ingredient of the wave-front tracking approximation procedure used in this paper.

Definition 6 (Constrained entropy solution) Let $\vec{W}_{0} \in \mathbf{B V}(\mathbb{R} ; \mathcal{W})$. A constrained weak solution $\vec{W} \in$ $\mathbf{L}^{\infty}(] 0,+\infty[; \mathbf{B V}(\mathbb{R} ; \mathcal{W})) \cap \mathbf{C}^{\mathbf{0}}\left(\mathbb{R}_{+} ; \mathbf{L}_{\text {loc }}^{\mathbf{1}}(\mathbb{R} ; \mathcal{W})\right)$ of $(3.4)$ in the sense of Definition 5 (hence satisfying (3.5)) is a constrained entropy solution if for any non-negative test function $\phi \in \mathbf{C}_{\mathbf{c}}^{\infty}(] 0,+\infty[\times \mathbb{R} ; \mathbb{R})$

$$
\begin{aligned}
& \int_{\mathbb{R}_{+}} \int_{\mathbb{R}}\left[\mathcal{E}_{k}(\vec{W}) \phi_{t}+\mathcal{Q}_{k}(\vec{W}) \phi_{x}\right] \mathrm{d} x \mathrm{~d} t \\
& +\int_{\mathbb{R}_{+}} \mathcal{N}_{k}\left(\vec{W}, q_{0}\right) \phi(t, 0) \mathrm{d} t \geq 0
\end{aligned}
$$

for any entropy pair (2.13) with $\left.k \in] 0, V_{0}\right]$, where

$$
\mathcal{N}_{k}\left(\vec{W}, q_{0}\right) \doteq \begin{cases}q(\vec{W}(t, 0))\left[\frac{k}{q_{0}(t)}-\frac{1}{p^{-1}([w(t, 0)-k]+)}\right]^{+} & \text {if } q_{0}(t) \neq 0 \\ k & \text { otherwise }\end{cases}
$$

The term $\mathcal{N}_{k}$ is designed to compensate the additional entropy dissipation at $x=0$ due to the effect of the constraint. One could obtain a similar compensation effect by using a term of a slightly different form, but the present formulation presents some advantages which will be apparent later in the paper. Some preliminary remarks on the meaning of (3.7) are in order. First of all, the traces $q\left(\vec{W}\left(t, 0^{ \pm}\right)\right)$and $w\left(t, 0^{ \pm}\right)$exist because $\vec{W}$ is in $\mathbf{L}^{\infty}\left(\mathbb{R}_{+} ; \mathbf{B V}(\mathbb{R} ; \mathcal{W})\right)$. The traces $w\left(t, 0^{-}\right)$and $w\left(t, 0^{+}\right)$may differ, however, in this case the entropy solution performs a stationary contact discontinuity at $x=0$ and therefore the integrand in line (3.6b) is equal to zero because $q\left(\vec{W}\left(t, 0^{-}\right)\right)=q\left(\vec{W}\left(t, 0^{+}\right)\right)=0$. Second, in the case $q_{0}(t)=0$, we also have $q\left(\vec{W}\left(t, 0^{ \pm}\right)\right)=0$ due to $(3.5)$. In this case, we choose to let $\mathcal{N}_{k}(\vec{W}, 0)=k$ to ensure the upper semi-continuity of the term (3.6b) w.r.t. the a.e. convergence of sequences of constraints $\left(q_{0}^{i}\right)_{i}$, which can be convenient for passage-to-the-limit arguments (see Remark 6).

Remark 5 In fact, it is possible to give sense to (3.6b) for every weak solution of (2.6), i.e., without assuming the BV regularity in space. To this aim, the integrand function of (3.6b) has to be understood as the weak normal trace of the appropriately chosen $\mathbf{L}^{\infty}$ divergence-measure field (see [14]). This amounts to define (3.6b) by the expression (3.9) introduced below (for time-dependent $q_{0}$, an additional term has to be added to account for a contribution of $\left.\partial_{t}\left[\frac{k}{q_{0}(t)}-\frac{1}{p^{-1}\left([w-k]^{+}\right)}\right]^{+}\right)$.

Let us explain now the role of $\mathcal{N}_{k}$ in $(3.6 \mathrm{~b})$ and the reasons why we choose this precise form for it. The introduction of this "compensation" term follows the idea of Colombo and Goatin [15] for the constrained LWR. Its role is to ensure that at $x=0$ the only admissible stationary discontinuities are the shocks corresponding to admissible fluxes $\left(q \leq q_{0}\right)$ and the non-classical shock corresponding to the flux $q_{0}$, see the last point in Proposition 4. For the constrained LWR, the simplest form for the compensation term is proposed in [13]. Let us stress that in both [13] and [15], the compensation term for the constrained LWR depends only on the parameter $k$ and $q_{0}$, but it does not depend on the solution itself. The situation for the constrained ARZ is more delicate. A straightforward calculation for the constrained ARZ shows that the natural compensation term in the entropy inequalities (3.6) takes the form

$$
\int_{\mathbb{R}_{+}}\left[k-\frac{q_{0}(t)}{p^{-1}\left([w(t, 0)-k]^{+}\right)}\right]^{+} \phi(t, 0) \mathrm{d} t .
$$

Unfortunately, it is not clear that an entropy formulation using the above term instead of (3.6b) is stable under a.e. convergence of solutions. At the same time, such stability property is a natural property of any reasonable notion of admissible solution; it is an essential ingredient of existence proofs that proceed by approximation, such as the proof given in Section 5 below. The following proposition shows that the formulation with compensation term (3.6b) can be recast in such a way that its stability w.r.t. strong convergence of solutions becomes evident. For simplicity, we will assume that $q_{0}$ is a constant independent of $t$. It is possible to deal with the case of $\mathbf{B V}$ time-dependent constraint $q_{0}(\cdot)$. The additional arguments required for this case are evoked in Remark 6 . 


\section{Proposition 3}

(i) For any weak solution $\vec{W}$ of $(2.6)$ in the sense of Definition 2 , any test function $\phi \in \mathbf{C}_{\mathbf{c}}^{\infty}(] 0,+\infty[\times \mathbb{R} ; \mathbb{R})$ and any constant $q_{0}>0$, the integral term in (3.6b) can be rewritten as follows

$$
\begin{aligned}
& \int_{\mathbb{R}_{+}} q(\vec{W}(t, 0))\left[\frac{k}{q_{0}}-\frac{1}{p^{-1}\left([w(t, 0)-k]^{+}\right)}\right]^{+} \phi(t, 0) \mathrm{d} t \\
= & \int_{\mathbb{R}_{+}} \int_{\mathbb{R}_{-}}\left[\rho(\vec{W}) \mathfrak{f}_{k}(w) \psi_{t} \xi+q(\vec{W}) \mathfrak{f}_{k}(w) \psi \xi_{x}\right] \mathrm{d} x \mathrm{~d} t \\
= & -\int_{\mathbb{R}_{+}} \int_{\mathbb{R}_{+}}\left[\rho(\vec{W}) \mathfrak{f}_{k}(w) \psi_{t} \xi+q(\vec{W}) \mathfrak{f}_{k}(w) \phi \xi_{x}\right] \mathrm{d} x \mathrm{~d} t,
\end{aligned}
$$

where $\mathfrak{f}_{k}(w) \doteq\left[\frac{k}{q_{0}}-\frac{1}{p^{-1}\left([w-k]^{+}\right)}\right]^{+}, \psi(t) \doteq \phi(t, 0)$ and $\xi$ is an arbitrary $\mathbf{C}_{\mathbf{c}}^{\infty}$ test function such that $\xi(0)=1$.

(ii) Assume that $\left(\vec{W}^{h}\right)_{h}$ is a uniformly bounded sequence of entropy solutions of (3.4) in the sense of Definition 6 and corresponding to the constant constraints $\left(q_{0}\right)_{h}$. If $\left(q_{0}^{h}\right)_{h}$ converges to $q_{0}>0$ and $\left(\vec{W}^{h}\right)_{h}$ converges to $\vec{W}$ a.e. on $\mathbb{R}_{+} \times \mathbb{R}$, then $\vec{W}$ is an entropy solution of (3.4) in the sense of Definition 6 , corresponding to the constraint $q_{0}$.

Proof To prove (i), we exploit Remark 3 by considering (2.11) with nonlinearity $\mathfrak{f}_{k}$, multiplied by the test function $(t, x) \mapsto \psi(t) \xi(x)$ and integrated in $(t, x) \in \mathbb{R}_{+} \times \mathbb{R}_{-}$or in $(t, x) \in \mathbb{R}_{+} \times \mathbb{R}_{+}$(the equality between (3.9) and (3.10) is a consequence of the fact that each of these terms equals the term (3.8)). E.g., to get the equality between (3.8) and (3.9), one uses (2.11), the Green-Gauss formula

$$
\int_{\mathbb{R}_{+}}\left[q \mathfrak{f}_{k}(w)\right]\left(t, 0^{-}\right) \psi(t) \mathrm{d} t=\int_{\mathbb{R}_{-}} \int_{\mathbb{R}_{+}} \operatorname{div}_{(t, x)}\left[\left(\begin{array}{c}
\rho \\
q
\end{array}\right) \mathfrak{f}_{k}(w) \psi(t) \xi(x)\right] \mathrm{d} t \mathrm{~d} x
$$

and the existence of strong trace at $x=0^{-}$of $\vec{W}$, which gives sense to the left-hand side of the above formula (3.8).

Now we prove (ii). The integrands both in (3.6a) and in (3.9) are continuous in $\vec{W}$; the integrand in (3.9) is continuous also in $q_{0}$. Since all these integrands are uniformly bounded and compactly supported, it is enough to use the dominated convergence argument in order to pass to the limit, as $h$ goes to infinity, in the entropy formulation (3.6) (with (3.6b) represented by (3.9)) written for $\vec{W}^{h}, q_{0}^{h}$. Analogous argument based on the GreenGauss formula permits to pass to the limit in conditions (3.5) written for $\vec{W}^{h}, q_{0}^{h}$. We find then the corresponding entropy formulation for $\vec{W}, q_{0}$ at the limit.

Remark 6 Let us sketch how Proposition 3 (ii) works for a not too irregular time-dependent constraint function $q_{0}$. To avoid additional technicalities, let us assume that all entropy solutions in the sequence $\left(\vec{W}^{h}\right)_{h}$ correspond to the same (non-constant in time) constraint $q_{0}(\cdot)$, and also that there exists a constant $\alpha>0$ such that $q_{0} \geq \alpha$ a.e. on $\mathbb{R}_{+}$. Assume that there exists a sequence $\left(q_{0}^{j}\right)_{j}$ of piecewise constant functions such that

$$
\begin{aligned}
& 0<\alpha \leq q_{0}^{j}(\cdot) \leq q_{0}(\cdot) \text { for all } j \in \mathbb{N} \text { and } \\
& q_{0}^{j}(t) \text { converges to } q_{0}(t) \text { as } j \text { goes to infinity for a.e. } t>0 .
\end{aligned}
$$

Observe in particular that it is possible to approximate monotone functions by piecewise constant functions from above and from below, therefore the above approximation property is true for $\mathbf{B V}$ functions $q_{0}$ such that $\inf _{t \in \mathbb{R}_{+}} q_{0}(t)>0$. Consider the family of inequalities (3.6) corresponding to $\left(q_{0}^{j}\right)_{j}$. Since $\mathcal{N}_{k}$ is non-increasing w.r.t. $q_{0}$, each of these modified inequalities is a weaker condition than the original inequality (3.6). Now, one can switch from $q_{0}$ to $q_{0}^{j}$ in (3.6b), and split the integral (3.6b) into the sum of integrals over intervals of time where $q_{0}^{j}$ is constant. Each of these integrals passes to the limit, as $\left(\vec{W}^{h}\right)_{h}$ converges to $\vec{W}$, due to the argument of Proposition 3 (ii). In this way, given a sequence of solutions $\left(\vec{W}^{h}\right)_{h}$ with constraint $q_{0}$, one finds as in Proposition 3 (ii) that the limit $\vec{W}$ of $\left(\vec{W}^{h}\right)_{h}$ fulfils the inequalities (3.6) with constraints $\left(q_{0}^{j}\right)_{j}$, for every $j \in \mathbb{N}$. Finally, letting $j$ go to infinity one proves that $\vec{W}$ is a constrained entropy solution with the original constraint $q_{0}$; this is possible because $\mathcal{N}_{k}$ is continuous w.r.t. $q_{0}$. Notice that the upper-semicontinuity for the compensation term $\mathcal{N}_{k}$ given by (3.7) in Definition 6 permits to deal with the case where $q_{0}$ takes values close to or equal to zero.

Let us now give the basic properties of constrained entropy solutions.

Proposition 4 Let $\vec{W}$ be a constrained entropy solution of (3.4) in the sense of Definition 6. Then $\vec{Y}=Y(\vec{W})$ satisfies the following properties:

1. Any discontinuity of $\vec{Y}$ satisfies the Rankine-Hugoniot jump conditions.

2. Any discontinuity of $\vec{Y}$ away from the constraint is classical, i.e. satisfies the Lax entropy inequalities.

3. Non-classical discontinuities of $\vec{Y}$, see [32], may occur only at the constraint location $x=0$, and in this case the flow at $x=0$ is the maximal flow allowed by the constraint. Namely, if the entropy solution performs a non-classical discontinuity at time $t_{0}>0$, then $q\left(\vec{Y}\left(t_{0}, 0^{ \pm}\right)\right)=q_{0}\left(t_{0}\right)$. 
Proof The first two properties are obvious, so we only prove the last one. This property is evident if $q_{0}\left(t_{0}\right) \geq \dot{q}\left(V_{0}\right)$ or $q_{0}\left(t_{0}\right)=0$, due to $(3.5)$ and to the fact that $q(\vec{Y}) \geq 0$. Therefore we can assume that $\left.q_{0}\left(t_{0}\right) \in\right] 0, \dot{q}\left(V_{0}\right)[$ and that $\vec{W}$ satisfies (3.6) together with (3.5), and performs at time $t_{0}>0$ a (stationary) non-classical shock from $\vec{W}_{\ell} \doteq\left(w, v_{\ell}\right)^{T}$ to $\vec{W}_{r} \doteq\left(w, v_{r}\right)^{T}$, with $v_{\ell}<v_{r}$ and $q\left(\vec{W}_{\ell}\right)=q\left(\vec{W}_{r}\right) \doteq f \leq q_{0}$. We want to prove that then $f=q_{0}$. Consider the test function

$$
\phi(t, x) \doteq\left[\int_{|x|-\varepsilon}^{+\infty} \delta_{\varepsilon}(z) \mathrm{d} z\right]\left[\int_{t-t_{0}+\varepsilon}^{t-t_{0}+2 \varepsilon} \delta_{\varepsilon}(z) \mathrm{d} z\right],
$$

where $\delta_{\varepsilon}$ is a smooth approximation of the Dirac mass centred at $0^{+}, \delta_{0^{+}}^{D}$, namely

$$
\delta_{\varepsilon} \in \mathbf{C}_{\mathbf{c}}^{\infty}\left(\mathbb{R} ; \mathbb{R}_{+}\right), \varepsilon \in \mathbb{R}_{+}, \operatorname{supp}\left(\delta_{\varepsilon}\right) \subseteq[0, \varepsilon],\left\|\delta_{\varepsilon}\right\|_{\mathbf{L}^{1}(\mathbb{R} ; \mathbb{R})}=1, \delta_{\varepsilon} \rightarrow \delta_{0^{+}}^{D} .
$$

Observe that as $\varepsilon$ goes to zero

$$
\begin{aligned}
& \phi\left(t_{0}, x\right) \equiv 0 \rightarrow 0, \\
& \phi(t, 0)=\int_{t-t_{0}+\varepsilon}^{t-t_{0}+2 \varepsilon} \delta_{\varepsilon}(z) \mathrm{d} z \rightarrow \delta_{t_{0}^{-}}^{D}(t), \\
& \phi_{t}(t, x)=\left[\int_{|x|-\varepsilon}^{+\infty} \delta_{\varepsilon}(z) \mathrm{d} z\right]\left[\delta_{\varepsilon}\left(t-t_{0}+2 \varepsilon\right)-\delta_{\varepsilon}\left(t-t_{0}+\varepsilon\right)\right] \rightarrow 0, \\
& \chi_{\mathbb{R}_{ \pm}}(x) \phi_{x}(t, x) \rightarrow \mp \delta_{0^{ \pm}}^{D}(x) \delta_{t_{0}^{-}}^{D}(t) .
\end{aligned}
$$

Then by the entropy condition (3.6) we have that for all $k \in] \hat{v}\left(q_{0}\left(t_{0}\right), w\right), \check{v}\left(q_{0}\left(t_{0}\right), w\right)[\subseteq] v_{\ell}, v_{r}[$

$$
\begin{aligned}
& \mathcal{Q}_{k}\left(\vec{W}_{\ell}\right)-\mathcal{Q}_{k}\left(\vec{W}_{r}\right)+f\left[\frac{k}{q_{0}\left(t_{0}\right)}-\frac{1}{p^{-1}(w-k)}\right]^{+} \\
= & -\left[k-\frac{f}{p^{-1}(w-k)}\right]+f\left[\frac{k}{q_{0}\left(t_{0}\right)}-\frac{1}{p^{-1}(w-k)}\right]=\left[\frac{f}{q_{0}\left(t_{0}\right)}-1\right] k \geq 0 .
\end{aligned}
$$

Since $f \leq q_{0}\left(t_{0}\right)$, the above estimate implies that $f=q_{0}\left(t_{0}\right)$.

Let $\mathbf{P C}$ denote the set of piecewise constant functions with a finite number of jumps and $\mathbf{P C} \mathbf{C}_{\mathbf{l o c}}$ the set of functions locally in PC. The following theorem on existence of entropy solutions to the constrained Cauchy problem (3.4) with PC constraints is the main result of this paper. The proof is based on the wave-front tracking algorithm restarted at every time where $q_{0}$ experiences a jump. Observe that variations in $q_{0}$ may lead to a sharp increase in the total variation of the solutions, as already observed in [26] at the level of the Riemann problem. In this paper we limit our attention to $\mathbf{P C}$ constraints; extension to the $\mathbf{P} \mathbf{C}_{\mathbf{l o c}}$ case is straightforward. In the general case, it is always possible to construct solutions corresponding to discretized versions of the constraint. However, the tools we introduce in this paper fail to control the variation of these solutions uniformly with respect to the parameter of discretization of $q_{0}$ without imposing unnatural assumptions on the ranges of $q_{0}$ and $\vec{W}_{0}$. Therefore existence and stability properties of solutions for ARZ with general time-dependent constraint are still open questions, to be addressed with a different approach.

Theorem 1 Let $\vec{W}_{0} \in \mathbf{B V}(\mathbb{R} ; \mathcal{W})$ satisfy $(2.15)$ and $q_{0} \in \mathbf{P C}\left(\mathbb{R}_{+} ;\left[0, \dot{q}\left(V_{0}\right)\right]\right)$ be such that

$$
\begin{aligned}
& x \in \mathbb{R}_{-} \mapsto J\left(q_{0}(0), w_{0}(x)\right) \in \mathbb{R}_{+} \text {has bounded total variation and } \\
& \left.\left.\left.\left.K_{0} \doteq \sum_{\substack{t>0 \text { s.t. } \\
q_{0}(t-) \neq q_{0}(t+)}}\left[\sup _{y \in \mathbb{R}_{-}} \mid \operatorname{TV}\left(J\left(q_{0}(t-), w_{0}\right) ;\right]-\infty, y\right]\right)-\operatorname{TV}\left(J\left(q_{0}(t+), w_{0}\right) ;\right]-\infty, y\right]\right) \mid\right] \text { is bounded, }
\end{aligned}
$$

where $J$ is defined in (3.3). Then the Cauchy problem (3.4) with initial datum $\vec{W}_{0}$ and constraint $q_{0}$ admits a constrained entropy solution $\vec{W} \in \mathbf{C}^{\mathbf{0}}\left(\mathbb{R}_{+} ; \mathbf{B V}(\mathbb{R} ; \mathcal{W})\right)$ in the sense of Definition 6 and for all $t, s \in \mathbb{R}_{+}$it satisfies

$$
\operatorname{TV}(\vec{W}(t)) \leq C, \quad\|\vec{W}(t)-\vec{W}(s)\|_{1} \leq L|t-s|, \quad \quad\|\vec{W}(t)\|_{\infty} \leq V_{0}
$$

where

$$
\begin{aligned}
& V_{0} \doteq\left\|\vec{W}_{0}\right\|_{\infty}, \\
& C \doteq \operatorname{TV}\left(\vec{W}_{0}\right)+3 \operatorname{TV}\left(J\left(q_{0}(0), w_{0}\right) ; \mathbb{R}_{-}\right)+2 V_{0}+3\left(K_{1}+K_{2}\right), \\
& L \doteq C \max \left\{V_{0}, p^{-1}\left(V_{0}\right) p^{\prime}\left(p^{-1}\left(V_{0}\right)\right)\right\} .
\end{aligned}
$$

Above, $K_{1}$ and $K_{2}$ are constants that may depend on $K_{0}$ (see Section 6 for more details). 
As in $[1,3]$, the proof of the above theorem is based on the wave-front tracking algorithm, see [15,19]. The details of the proof are deferred to Section 6; the main ingredient is the choice of a Temple functional allowing to prove the following result.

Proposition 5 Let $\vec{W}_{0} \in \mathbf{B V}(\mathbb{R} ; \mathcal{W})$ satisfy $(2.15)$ and $q_{0} \geq 0$ be a constant such that $x \mapsto J\left(q_{0}, w_{0}(x)\right)$ has bounded total variation, where $J$ is defined in (3.3). Then the Cauchy problem (3.4) with initial datum $\vec{W}_{0}$ and constant constraint $q_{0}$ admits a constrained entropy solution $\vec{W} \in \mathbf{C}^{\mathbf{0}}\left(\mathbb{R}_{+} ; \mathbf{B V}(\mathbb{R} ; \mathcal{W})\right)$ in the sense of Definition 6 and for all $t, s \in \mathbb{R}_{+}$it satisfies

$$
\operatorname{TV}(\vec{W}(t)) \leq C, \quad\|\vec{W}(t)-\vec{W}(s)\|_{1} \leq L|t-s|, \quad \quad\|\vec{W}(t)\|_{\infty} \leq V_{0},
$$

where

$$
V_{0} \doteq\left\|\vec{W}_{0}\right\|_{\infty}, \quad C \doteq \mathrm{TV}\left(\vec{W}_{0}\right)+3 \mathrm{TV}\left(J\left(q_{0}, w_{0}\right) ; \mathbb{R}_{-}\right)+2 V_{0}, \quad L \doteq C \max \left\{V_{0}, p^{-1}\left(V_{0}\right) p^{\prime}\left(p^{-1}\left(V_{0}\right)\right)\right\}
$$

The proof is postponed to Section 5. Let us mention in passing that the dependence of the Temple functional on the constraint level $q_{0}$, via the map $J\left(q_{0}, \cdot\right)$, is the key obstacle for generalizing the existence result to general time-dependent $q_{0}$; the difficulty comes from the singularity observed in Lemma 2 . Let us stress that in the easier case of LWR subject to point constraints it is possible to use a Temple functional independent from the constraint level.

\section{Example}

In this section we apply the model (3.4) to simulate the traffic on a road in presence of a traffic light placed at $x=0$. The simulation is obtained by explicit analysis of the wave-front interactions, with computer-assisted computation of front slopes and interaction times presented in Figure 2, right. While the overall picture of the corresponding solution is rather stable, a detailed analytical study necessarily needs to consider many slightly different cases. Below, we restrict the construction of the solution to the most representative situation.
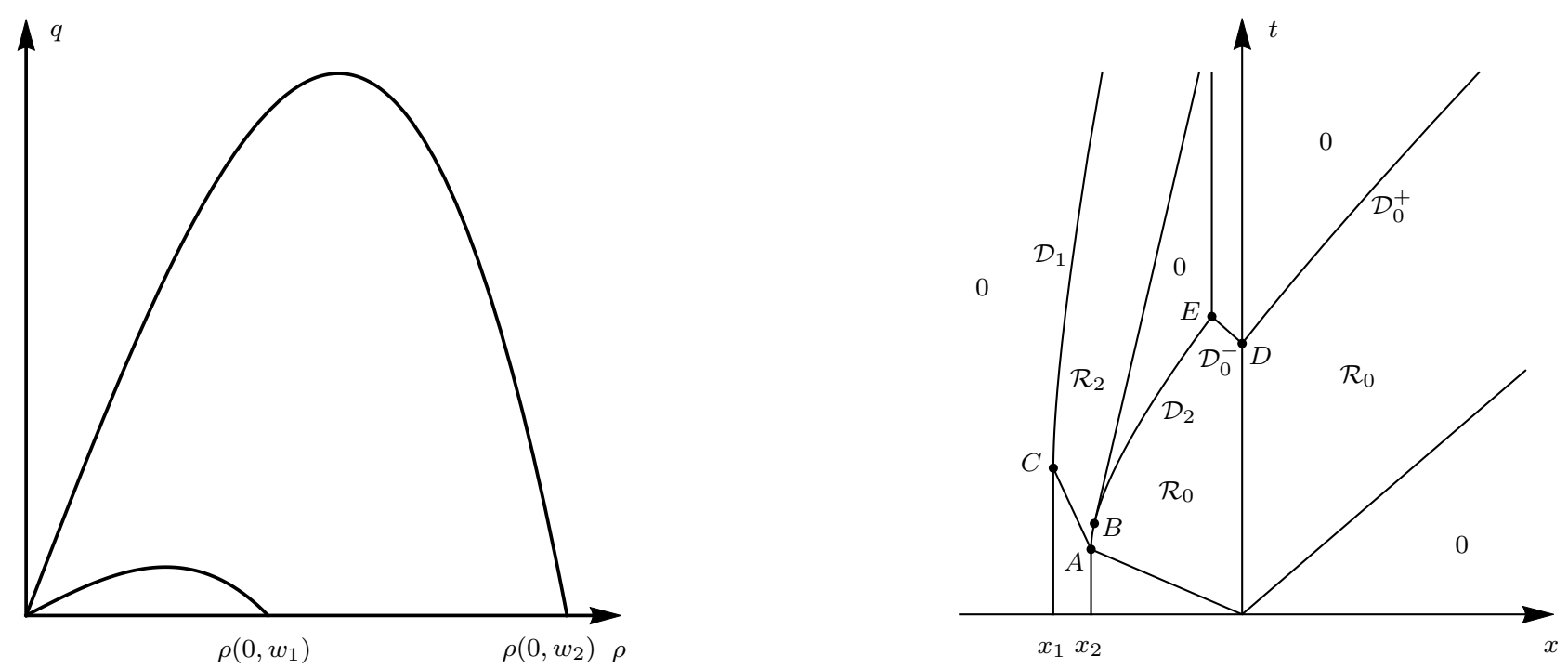

Fig. 2 With reference to Section 4, on the left the two fundamental graphics corresponding to the two Lagrangian markers $w_{1}$ and $w_{2}$, on the right the representation of the solution in the $(x, t)$-plane. In the figure on the right, the zeros stand for the vacuum.

Consider two types of vehicles, the "slow vehicles" and "fast vehicles", characterized by the Lagrangian markers $w_{1}$ and $w_{2}$, respectively, with $0<w_{1}<w_{2}$. With this choice, the slow vehicles have maximal speed $w_{1}$ lower than the maximal speed $w_{2}$ of the fast vehicles. Observe also that the slow vehicles have length $1 / \rho\left(0, w_{1}\right)$ bigger than the length $1 / \rho\left(0, w_{2}\right)$ of the fast vehicles.

Assume that all the vehicles are initially at rest in $\left[x_{1}, 0[\right.$. More precisely, assume that the slow vehicles are uniformly distributed in $\left[x_{1}, x_{2}\right.$ [ with density $\rho\left(0, w_{1}\right)$, and that the fast vehicles are uniformly distributed in $\left[x_{2}, 0[\right.$ with density $\rho\left(0, w_{2}\right)$, see the first line of Figure 3. At time $t=0$ the traffic light turns to green and the first vehicle from the right starts to move. Hence, the solution performs two stationary discontinuities $\mathcal{D}_{1}$ at $x=x_{1}$ 

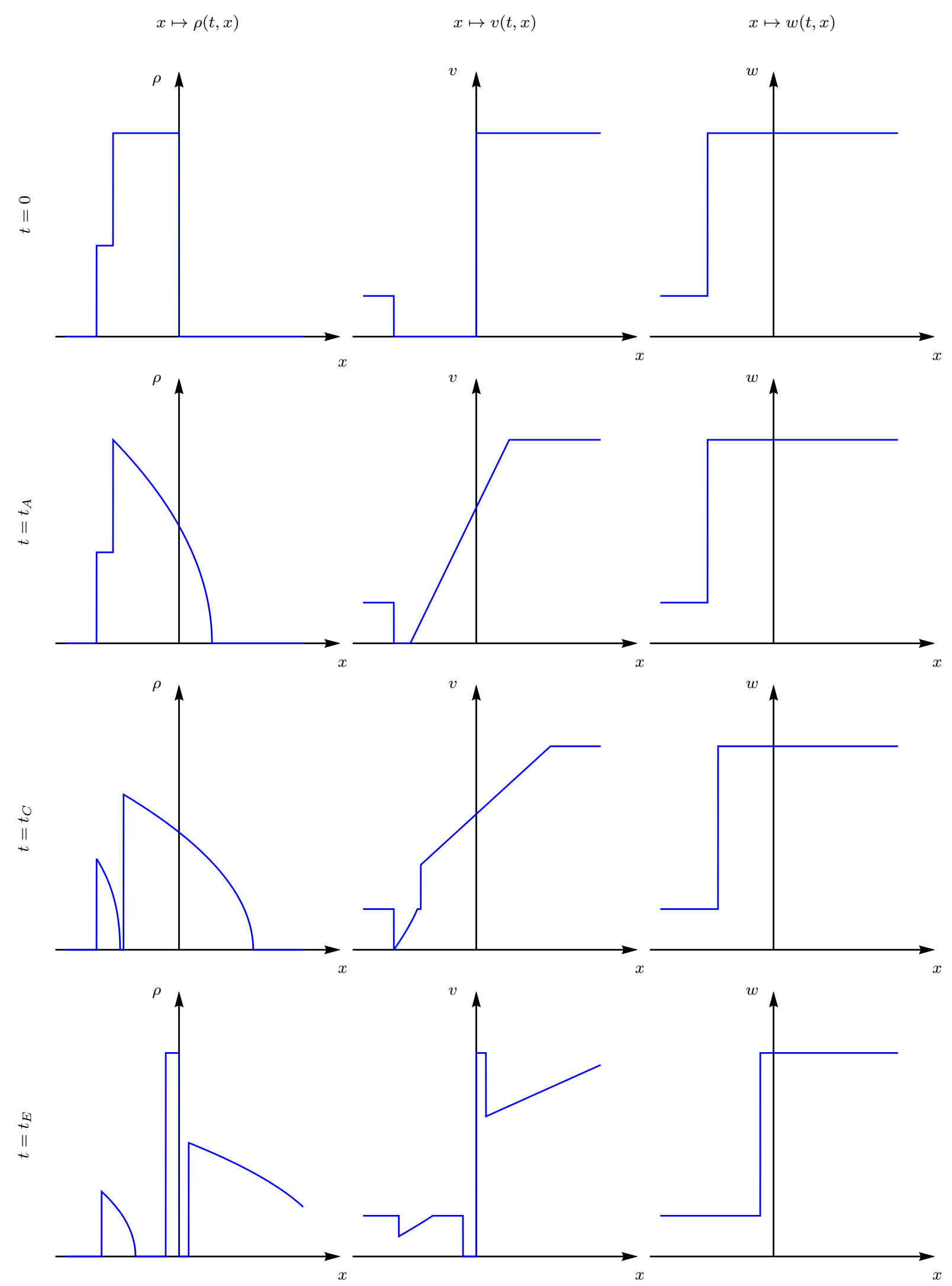

Fig. 3 Starting from the left, respectively, density $\rho$, velocity $v$ and Lagrangian marker $w$ profiles at times $t=0$ (first line), $t=t_{A}$ (second line), $t=t_{C}$ (third line) and $t=t_{E}$ (last line). 
and $\mathcal{D}_{2}$ at $x=x_{2}$, followed by a rarefaction $\mathcal{R}_{0}$ centred at $x=0$. For times sufficiently small, the expression of the solution is therefore

$$
\vec{W}(t, x)= \begin{cases}\left(w_{1}, w_{1}\right)^{T} & \text { if } x<x_{1} \\ \left(0, w_{1}\right)^{T} & \text { if } x_{1} \leq x<x_{2} \\ \left(0, w_{2}\right)^{T} & \text { if } x_{2} \leq x<\lambda_{1}\left(0, w_{2}\right) t \\ \left(w_{2}-p\left(\Pi\left(w_{2}-\frac{x}{t}\right)\right), w_{2}\right)^{T} & \text { if } \lambda_{1}\left(0, w_{2}\right) t \leq x<\lambda_{1}\left(w_{2}, w_{2}\right) t \\ \left(w_{2}, w_{2}\right)^{T} & \text { if } x \geq \lambda_{1}\left(w_{2}, w_{2}\right) t\end{cases}
$$

where $\Pi$ is the inverse function of $\rho \mapsto p(\rho)+\rho p^{\prime}(\rho)$. The above expression holds true until all the slow vehicles are stopped, namely, for $\left.t \in] 0, t_{A}\right]$, where $t_{A} \doteq x_{2} / \lambda_{1}\left(0, w_{2}\right)$.

At time $t=t_{A}$, the rarefaction $\mathcal{R}_{0}$ reaches the stationary contact discontinuity $\mathcal{D}_{2}$ and the first slow vehicle from the right starts to move. As a result of the interaction, from $A \doteq\left(x_{2}, t_{A}\right)$ starts a rarefaction $\mathcal{R}_{2}$, that for small times is contained in $\left\{(x, t) \in \mathbb{R} \times \mathbb{R}_{+}: t>t_{A}\right.$ and $\left.x_{2}+\lambda_{1}\left(0, w_{1}\right)\left(t-t_{A}\right)<x<\mathfrak{D}_{2}(t)\right\}$, followed by the contact discontinuity $\mathcal{D}_{2}$, given by the support of $\mathfrak{D}_{2}$, that is the solution of the ODE

$$
\frac{\mathrm{d} \mathfrak{D}_{2}}{\mathrm{~d} t}(t)=w_{2}-p\left(\Pi\left(w_{2}-\frac{\mathfrak{D}_{2}(t)}{t}\right)\right), \quad \mathfrak{D}_{2}\left(t_{A}\right)=x_{2} .
$$

Due to the interaction with the rarefaction $\mathcal{R}_{0}$, the contact discontinuity $\mathcal{D}_{2}$ accelerates and on its left new rarefactions (still denoted $\mathcal{R}_{2}$ ) appear.

By applying the method of characteristics, we find the values attained by the solution in $\mathcal{R}_{2}$. More precisely, for any $t>t_{A}$ sufficiently small and $x \in\left[x_{2}+\lambda_{1}\left(0, w_{1}\right)\left(t-t_{A}\right), \mathfrak{D}_{2}(t)\right]$ such that $(x, t) \in \mathcal{R}_{2}$, we define $(t, x) \mapsto$ $\left(X_{1}(t, x), T_{1}(t, x)\right)$ as the projection of $(t, x)$ on $\mathcal{D}_{2}$ along the characteristics of the first family, namely

$$
X_{1}(t, x)=\mathfrak{D}_{2}\left(T_{1}(t, x)\right), \quad X_{1}(t, x)+\lambda_{1}\left(w_{2}-p\left(\Pi\left(w_{2}-\frac{X_{1}(t, x)}{T_{1}(t, x)}\right)\right), w_{1}\right)\left(t-T_{1}(t, x)\right)=x .
$$

Then, for $t>t_{A}$ sufficiently small, the expression of the solution is

$$
\vec{W}(t, x)= \begin{cases}\left(w_{1}, w_{1}\right)^{T} & \text { if } x<x_{1}, \\ \left(0, w_{1}\right)^{T} & \text { if } x_{1} \leq x<x_{2}+\lambda_{1}\left(0, w_{1}\right)\left(t-t_{A}\right), \\ \left(w_{2}-p\left(\Pi\left(w_{2}-\frac{X_{1}(t, x)}{T_{1}(t, x)}\right)\right), w_{1}\right)^{T} & \text { if } x_{2}+\lambda_{1}\left(0, w_{1}\right)\left(t-t_{A}\right) \leq x<\mathfrak{D}_{2}(t), \\ \left(w_{2}-p\left(\Pi\left(w_{2}-\frac{x}{t}\right)\right), w_{2}\right)^{T} & \text { if } \mathfrak{D}_{2}(t) \leq x<\lambda_{1}\left(w_{2}, w_{2}\right) t \\ \left(w_{2}, w_{2}\right)^{T} & \text { if } x \geq \lambda_{1}\left(w_{2}, w_{2}\right) t\end{cases}
$$

At time $t_{B}$ implicitly given by

$$
w_{2}-p\left(\Pi\left(w_{2}-\frac{\mathfrak{D}_{2}\left(t_{B}\right)}{t_{B}}\right)\right)=w_{1}
$$

the vacuum between the two types of vehicles appears. In fact, for $t>t_{B}$ sufficiently small, the vacuum state $\left(w_{1}, w_{1}\right) \in \mathcal{W}_{0}$ appears between the rarefaction $\mathcal{R}_{2}$ and the contact discontinuity $\mathcal{D}_{2}$.

At time $t_{C} \doteq t_{A}+\left[x_{1}-x_{2}\right] / \lambda_{1}\left(0, w_{1}\right)$ all the slow vehicles are moving because the stationary discontinuity $\mathcal{D}_{1}$ is reached at $C \doteq\left(x_{1}, t_{C}\right)$ by the rarefaction $\mathcal{R}_{2}$. As a result of the interaction with $\mathcal{R}_{2}$, the discontinuity $\mathcal{D}_{1}$ starts to accelerate with a support given by $\mathfrak{D}_{1}$, that is the solution of the ODE

$$
\frac{\mathrm{d} \mathfrak{D}_{1}}{\mathrm{~d} t}(t)=w_{2}-p\left(\Pi\left(w_{2}-\frac{X_{1}\left(t, \mathfrak{D}_{1}(t)\right)}{T_{1}\left(t, \mathfrak{D}_{1}(t)\right)}\right)\right), \quad \mathfrak{D}_{1}\left(t_{C}\right)=x_{1} .
$$

For simplicity, assume that $t_{B}<t_{C}$, then the solution for $\left.\left.t \in\right] t_{B}, t_{C}\right]$ has the expression

$$
\vec{W}(t, x)= \begin{cases}\left(w_{1}, w_{1}\right)^{T} & \text { if } x<x_{1}, \\ \left(0, w_{1}\right)^{T} & \text { if } x_{1} \leq x<x_{2}+\lambda_{1}\left(0, w_{1}\right)\left(t-t_{A}\right), \\ \left(w_{2}-p\left(\Pi\left(w_{2}-\frac{X_{1}(t, x)}{T_{1}(t, x)}\right)\right), w_{1}\right)^{T} & \text { if } x_{2}+\lambda_{1}\left(0, w_{1}\right)\left(t-t_{A}\right) \leq x<\mathfrak{D}_{2}\left(t_{B}\right)+w_{1}\left(t-t_{B}\right), \\ \left(w_{1}, w_{1}\right)^{T} & \text { if } \mathfrak{D}_{2}\left(t_{B}\right)+w_{1}\left(t-t_{B}\right) \leq x<\mathfrak{D}_{2}(t) \\ \left(w_{2}-p\left(\Pi\left(w_{2}-\frac{x}{t}\right)\right), w_{2}\right)^{T} & \text { if } \mathfrak{D}_{2}(t) \leq x<\lambda_{1}\left(w_{2}, w_{2}\right) t \\ \left(w_{2}, w_{2}\right)^{T} & \text { if } x \geq \lambda_{1}\left(w_{2}, w_{2}\right) t\end{cases}
$$


and for $t>t_{C}$ sufficiently small

$$
\vec{W}(t, x)= \begin{cases}\left(w_{1}, w_{1}\right)^{T} & \text { if } x<\mathfrak{D}_{1}(t), \\ \left(w_{2}-p\left(\Pi\left(w_{2}-\frac{X_{1}(t, x)}{T_{1}(t, x)}\right), w_{1}\right)^{T}\right. & \text { if } \mathfrak{D}_{1}(t) \leq x<\mathfrak{D}_{2}\left(t_{B}\right)+w_{1}\left(t-t_{B}\right), \\ \left(w_{1}, w_{1}\right)^{T} & \text { if } \mathfrak{D}_{2}\left(t_{B}\right)+w_{1}\left(t-t_{B}\right) \leq x<\mathfrak{D}_{2}(t), \\ \left(w_{2}-p\left(\Pi\left(w_{2}-\frac{x}{t}\right)\right), w_{2}\right)^{T} & \text { if } \mathfrak{D}_{2}(t) \leq x<\lambda_{1}\left(w_{2}, w_{2}\right) t, \\ \left(w_{2}, w_{2}\right)^{T} & \text { if } x \geq \lambda_{1}\left(w_{2}, w_{2}\right) t .\end{cases}
$$

Assume that at time $t=t_{D}$ the traffic light turns again to red. Assume also that $\mathfrak{D}_{2}\left(t_{D}\right)<0$. Then in $\mathbb{R}_{-}$there are still vehicles, that form a queue that grows with time. The tail of such queue is identified by the backward shock $\mathcal{D}_{0}^{-}$. Beside $\mathcal{D}_{0}^{-}$, from $D \doteq\left(0, t_{D}\right)$ start a stationary non-classical shock and a forward shock $\mathcal{D}_{0}^{+}$. More precisely, $\mathcal{D}_{0}^{ \pm}$is the support of $\mathfrak{D}_{0}^{ \pm}$, where $\mathfrak{D}_{0}^{ \pm}$is the solution of the corresponding ODE

$$
\begin{array}{ll}
\frac{\mathrm{d} \mathfrak{D}_{0}^{-}}{\mathrm{d} t}(t)=\sigma\left(w_{2}-p\left(\Pi\left(w_{2}-\frac{x}{t}\right)\right), w_{2}, 0, w_{2}\right), & \mathfrak{D}_{0}^{-}\left(t_{D}\right)=0, \\
\frac{\mathrm{d} \mathfrak{D}_{0}^{+}}{\mathrm{d} t}(t)=w_{2}-p\left(\Pi\left(w_{2}-\frac{x}{t}\right)\right), & \mathfrak{D}_{0}^{+}\left(t_{D}\right)=0 .
\end{array}
$$

If the intersection of $\mathcal{D}_{2}$ with $\mathcal{D}_{0}^{-}$is denoted by $E$, then $E$ is implicitly given by

$$
\mathfrak{D}_{2}\left(t_{E}\right)=x_{E}=\mathfrak{D}_{0}^{-}\left(t_{E}\right) \text {. }
$$

The expression of the solution for $\left.t \in] t_{D}, t_{E}\right]$ is then

$$
\vec{W}(t, x)= \begin{cases}\left(w_{1}, w_{1}\right)^{T} & \text { if } x<\mathfrak{D}_{1}(t), \\ \left(w_{2}-p\left(\Pi\left(w_{2}-\frac{X_{1}(t, x)}{T_{1}(t, x)}\right)\right), w_{1}\right)^{T} & \text { if } \mathfrak{D}_{1}(t) \leq x<\mathfrak{D}_{2}\left(t_{B}\right)+w_{1}\left(t-t_{B}\right), \\ \left(w_{1}, w_{1}\right)^{T} & \text { if } \mathfrak{D}_{2}\left(t_{B}\right)+w_{1}\left(t-t_{B}\right) \leq x<\mathfrak{D}_{2}(t), \\ \left(w_{2}-p\left(\Pi\left(w_{2}-\frac{x}{t}\right)\right), w_{2}\right)^{T} & \text { if } \mathfrak{D}_{2}(t) \leq x<\mathfrak{D}_{0}^{-}(t), \\ \left(0, w_{2}\right)^{T} & \text { if } \mathfrak{D}_{0}^{-}(t) \leq x<0, \\ \left(w_{2}, w_{2}\right)^{T} & \text { if } 0 \leq x<\mathfrak{D}_{0}^{+}(t), \\ \left(w_{2}-p\left(\Pi\left(w_{2}-\frac{x}{t}\right)\right), w_{2}\right)^{T} & \text { if } \mathfrak{D}_{0}^{+}(t) \leq x<\lambda_{1}\left(w_{2}, w_{2}\right) t, \\ \left(w_{2}, w_{2}\right)^{T} & \text { if } x \geq \lambda_{1}\left(w_{2}, w_{2}\right) t .\end{cases}
$$

At time $t=t_{E}$, the queue stops to grow, the contact discontinuity $\mathcal{D}_{2}$ becomes stationary and the expression of the solution for $t>t_{E}$ sufficiently small is

$$
\vec{W}(t, x)= \begin{cases}\left(w_{1}, w_{1}\right)^{T} & \text { if } x<\mathfrak{D}_{1}(t), \\ \left(w_{2}-p\left(\Pi\left(w_{2}-\frac{X_{1}(t, x)}{T_{1}(t, x)}\right)\right), w_{1}\right)^{T} & \text { if } \mathfrak{D}_{1}(t) \leq x<\mathfrak{D}_{2}\left(t_{B}\right)+w_{1}\left(t-t_{B}\right), \\ \left(w_{1}, w_{1}\right)^{T} & \text { if } \mathfrak{D}_{2}\left(t_{B}\right)+w_{1}\left(t-t_{B}\right) \leq x<x_{E}, \\ \left(0, w_{2}\right)^{T} & \text { if } x_{E} \leq x<0, \\ \left(w_{2}, w_{2}\right)^{T} & \text { if } 0 \leq x<\mathfrak{D}_{0}^{+}(t), \\ \left(w_{2}-p\left(\Pi\left(w_{2}-\frac{x}{t}\right)\right), w_{2}\right)^{T} & \text { if } \mathfrak{D}_{0}^{+}(t) \leq x<\lambda_{1}\left(w_{2}, w_{2}\right) t, \\ \left(w_{2}, w_{2}\right)^{T} & \text { if } x \geq \lambda_{1}\left(w_{2}, w_{2}\right) t .\end{cases}
$$

Clearly, the solution can be constructed for all times. However, we stop the description of its construction to avoid to overload the paper and because the remaining interactions are similar to those already considered in the previous steps.

\section{The Cauchy problem with constant constraint: Proof of Proposition 5}

In this section we apply the wave-front tracking algorithm to construct a sequence $\left(\vec{W}^{h}\right)_{h}$ that converges (along a subsequence) to an entropy solution of the Cauchy problem (3.4) for a constant constraint $q_{0}$; thus, we prove Proposition 5. The case $q_{0}=0$ reduces (3.4) to two decoupled initial-boundary value problems, one in $\mathbb{R}_{-}$with boundary datum $v(t, 0)=0$ and one in $\mathbb{R}_{+}$with boundary condition $\rho(t, 0)=0$, and then it is sufficient to apply the standard theory developed in [6]. In the following subsections we therefore describe the construction of $\vec{W}^{h}$ in full details in the case $q_{0}>0$. 
5.1 Approximation of initial datum and constraint

Fix $h \in \mathbb{N}$ sufficiently large and take $\varepsilon^{h} \doteq 2^{-h} V_{0}$, with $V_{0} \doteq\left\|\vec{W}_{0}\right\|_{\infty}$.
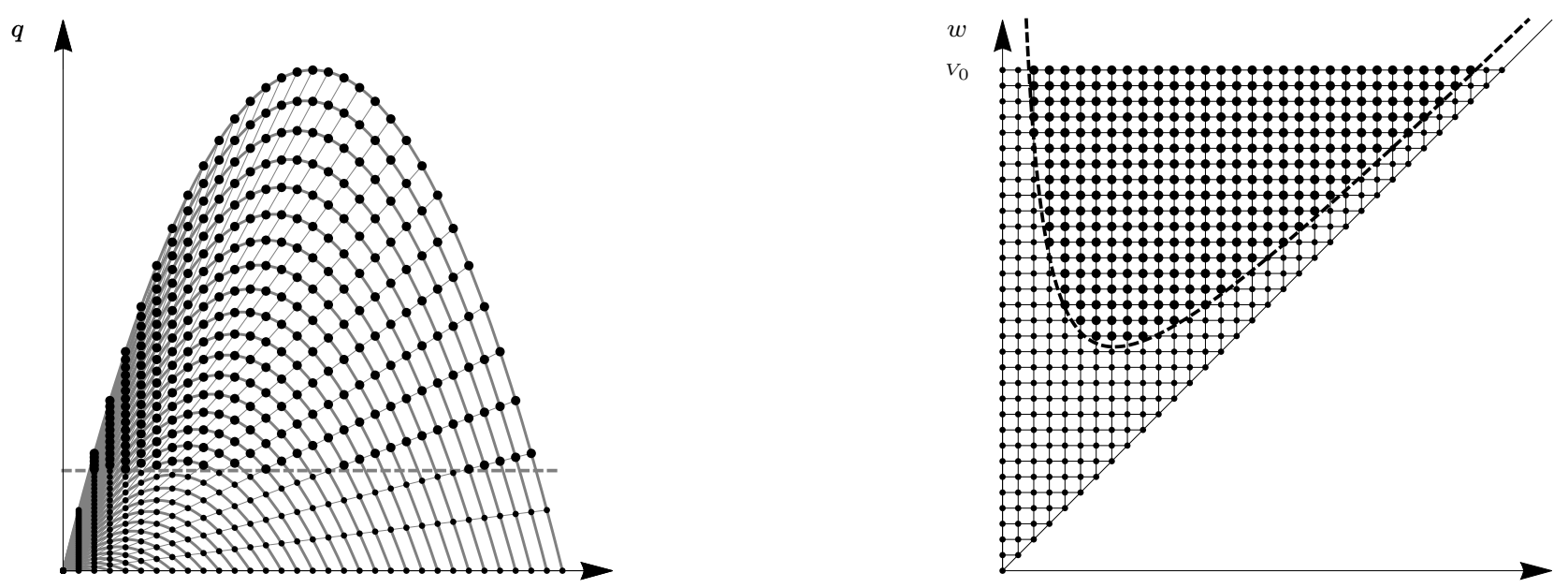

Fig. 4 With reference to Section 5.1, the dots represent the grid $\mathcal{W}^{h}$, the bigger dots are the elements of $\mathcal{C}^{h}$, the dashed curve is boundary of $\mathcal{C}$ in the $(\rho, q)$-plane on the left, and in the $(v, w)$-plane on the right. $q_{0}>0$

Introduce the grid $\mathcal{W}^{h} \doteq \mathcal{W} \cap\left[\varepsilon^{h} \mathbb{N}^{2}\right]$ and approximate the constrained states corresponding to the constraint

with, see Figure 4,

$$
\mathcal{C}\left(q_{0}\right) \doteq\left\{(v, w)^{T} \in \mathcal{W}: w>v+p\left(\frac{q_{0}}{v}\right)\right\}
$$

$$
\mathcal{C}^{h}\left(q_{0}\right) \doteq\left\{(v, w)^{T} \in \mathcal{W}^{h}: w>v+p\left(\frac{q_{0}}{v}\right)\right\}
$$

For any $w \geq 0$ introduce

$$
\hat{v}^{h}\left(q_{0}, w\right) \doteq \max \left\{\varepsilon^{h} \mathbb{N} \cap\left[0, \hat{v}\left(q_{0}, w\right)\right]\right\}, \quad \quad \check{v}^{h}\left(q_{0}, w\right) \doteq \max \left\{\varepsilon^{h} \mathbb{N} \cap\left[0, \check{v}\left(q_{0}, w\right)\right]\right\},
$$

where $\hat{v}$ and $\check{v}$ are given in (3.2), and in analogy to (3.3) define

$$
J^{h} \doteq \check{v}^{h}-\hat{v}^{h}
$$

If we let

$$
\tilde{w}^{h}\left(q_{0}\right) \doteq \min \left\{w \in \varepsilon^{h} \mathbb{N}: \mathcal{C}^{h}\left(q_{0}\right) \cap([0, w] \times\{w\}) \text { has at least two elements }\right\},
$$

then, by definition, for any $w \in\left[\tilde{w}^{h}\left(q_{0}\right),+\infty\left[\cap \varepsilon^{h} \mathbb{N}\right.\right.$ we have

$$
\begin{gathered}
0 \leq \hat{v}\left(q_{0}, w\right)-\hat{v}^{h}\left(q_{0}, w\right)<\varepsilon^{h}, \quad 0 \leq \check{v}\left(q_{0}, w\right)-\check{v}^{h}\left(q_{0}, w\right)<\varepsilon^{h}, \\
q\left(\hat{W}^{h}\left(q_{0}, w\right)\right) \leq q_{0} \leq q\left(\check{W}^{h}\left(q_{0}, w\right)\right), \\
\lim _{h \rightarrow+\infty} q\left(\check{W}^{h}\left(q_{0}, w\right)\right)=\lim _{h \rightarrow+\infty} q\left(\hat{W}^{h}\left(q_{0}, w\right)\right)=q_{0}, \\
\check{v}^{h}\left(q_{0}, w\right)-\hat{v}^{h}\left(q_{0}, w\right) \geq 2 \varepsilon^{h},
\end{gathered}
$$

where $\hat{W}^{h}\left(q_{0}, w\right) \doteq\left(\hat{v}^{h}\left(q_{0}, w\right), w\right)^{T}, \check{W}^{h}\left(q_{0}, w\right) \doteq\left(\check{v}^{h}\left(q_{0}, w\right), w\right)^{T}$ and by Lemma 2

$$
\check{v}^{h}\left(q_{0}, w+\varepsilon^{h}\right)-\hat{v}^{h}\left(q_{0}, w+\varepsilon^{h}\right) \neq \check{v}^{h}\left(q_{0}, w\right)-\hat{v}^{h}\left(q_{0}, w\right) .
$$

Approximate $\vec{W}_{0}$ with $\vec{W}_{0}^{h} \in \mathbf{P C}\left(\mathbb{R} ; \mathcal{W}^{h}\right)$ satisfying $(2.15)$ and such that for some $C=C\left(\mathrm{TV}\left(J\left(q_{0}, w_{0}\right) ; \mathbb{R}_{-}\right)\right)$ independent of $h$, there holds

$$
\begin{gathered}
\left\|\vec{W}_{0}^{h}\right\|_{\infty} \leq V_{0}, \quad \lim _{h \rightarrow+\infty}\left\|\vec{W}_{0}-\vec{W}_{0}^{h}\right\|_{1}=0, \quad \operatorname{TV}\left(\vec{W}_{0}^{h}\right) \leq \operatorname{TV}\left(\vec{W}_{0}\right) \\
\operatorname{TV}\left(J^{h}\left(q_{0}, w_{0}^{h}\right) ; \mathbb{R}_{-}\right) \leq C\left(\operatorname{TV}\left(J\left(q_{0}, w_{0}\right) ; \mathbb{R}_{-}\right)\right) .
\end{gathered}
$$


Then we obtain the approximate constrained Cauchy problem

$$
\begin{array}{ll}
Y(\vec{W})_{t}+F(Y(\vec{W}))_{x}=0, & t>0, x \in \mathbb{R}, \\
\vec{W}(0, x)=\vec{W}_{0}^{h}(x), & x \in \mathbb{R}, \\
\vec{W}\left(t, 0^{ \pm}\right) \notin \mathcal{C}^{h}\left(q_{0}\right), & t>0 .
\end{array}
$$

Let $\mathcal{R} \mathcal{S}^{h}$, respectively $\mathcal{R S}_{q_{0}}^{h}$, be the approximate Riemann solver of (5.5a), (5.5b), respectively the approximate constrained Riemann solver of (5.5), both described in details in Section 5.2. The approximate solution $\vec{W}^{h}$ to (5.5) is then obtained by gluing together the approximate solutions computed by applying $\mathcal{R S}_{q_{0}}^{h}$ in $x=0$ at time $t=0$ and at any time a wave front reaches $x=0$, and by applying $\mathcal{R} \mathcal{S}^{h}$ at any discontinuity of $\vec{W}_{0}^{h}$ away from $x=0$ or at any interaction between wave fronts away from $x=0$.

As usual, in order to extend the construction globally in time we have to ensure that only finitely many interactions may occur in finite time. In Section 5.3 we prove that $\vec{W}^{h}(t)$ is well defined for all $t>0$ and belongs to $\mathbf{P C}\left(\mathbb{R} ; \mathcal{W}^{h}\right)$. Finally, in Section 5.4 we prove that $\left(\vec{W}^{h}\right)_{h}$ converges (along a subsequence) in $\mathbf{L}_{\text {loc }}^{\mathbf{1}}$ to a limit $\vec{W}$ and prove that $\vec{W}$ is in fact a constrained entropy solution to (3.4) (with $q_{0}$ constant) in the sense of Definition 6 .

5.2 The approximate Riemann solvers

Let $\mathfrak{G}^{h} \doteq \mathfrak{G} \cap\left(\mathcal{W}^{h} \times \mathcal{W}^{h}\right)$. In this section we define the approximate Riemann solvers $\mathcal{R S}^{h}, \mathcal{R S}_{q_{0}}^{h}: \mathfrak{G}^{h} \rightarrow$ $\mathbf{C}^{\mathbf{0}}\left(\mathbb{R}_{+} ; \mathbf{L}_{\text {loc }}^{\mathbf{1}}\left(\mathbb{R} ; \mathcal{W}^{h}\right)\right)$ corresponding to $(5.5)$, respectively away from $x=0$ and at $x=0$.

First, $\mathcal{R S}^{h}$ is obtained by discretizing the rarefaction waves of $\mathcal{R S}$. More precisely, if $\left(\vec{W}_{\ell}, \vec{W}_{r}\right) \in \mathfrak{G}^{h}$ with $w_{\ell}=w_{r}$ and $v_{r}=v_{\ell}+j \varepsilon^{h}, j>0$, then

$$
\mathcal{R S}^{h}\left[\vec{W}_{\ell}, \vec{W}_{r}\right](x)= \begin{cases}\vec{W}_{\ell} & \text { if } x<\sigma\left(\vec{W}_{\ell}, \vec{W}_{1}\right), \\ \vec{W}_{i} & \text { if } \sigma\left(\vec{W}_{i-1}, \vec{W}_{i}\right)<x<\sigma\left(\vec{W}_{i}, \vec{W}_{i+1}\right), \\ \vec{W}_{r} & \text { if } x>\sigma\left(\vec{W}_{j-1}, \vec{W}_{r}\right),\end{cases}
$$

where $\vec{W}_{i} \doteq\left(v_{\ell}+i \varepsilon^{h}, w_{\ell}\right)^{T} \in \mathcal{W}^{h}$. Then, we define $\mathcal{R} \mathcal{S}_{q_{0}}^{h}$ as follows:

- if $\mathcal{R S}^{h}\left[\vec{W}_{\ell}, \vec{W}_{r}\right]$ satisfies $(5.5 \mathrm{c})$, then

$$
\mathcal{R S}_{q_{0}}^{h}\left[\vec{W}_{\ell}, \vec{W}_{r}\right] \equiv \mathcal{R} \mathcal{S}^{h}\left[\vec{W}_{\ell}, \vec{W}_{r}\right]
$$

- otherwise we let

$$
\mathcal{R S}_{q_{0}}^{h}\left[\vec{W}_{\ell}, \vec{W}_{r}\right](x / t)= \begin{cases}\mathcal{R} \mathcal{S}^{h}\left[\vec{W}_{\ell}, \hat{W}^{h}\left(q_{0}, w_{\ell}\right)\right](x / t), & x<0, \\ \mathcal{R} \mathcal{S}^{h}\left[\check{W}^{h}\left(q_{0}, w_{\ell}\right), \vec{W}_{r}\right](x / t), & x>0 .\end{cases}
$$

By construction both $x \mapsto \mathcal{R} \mathcal{S}^{h}\left[\vec{W}_{\ell}, \vec{W}_{r}\right](x)$ and $x \mapsto \mathcal{R} \mathcal{S}_{q_{0}}^{h}\left[\vec{W}_{\ell}, \vec{W}_{r}\right](x)$ take values in $\mathcal{W}^{h}$ and, by Lemma 1 , satisfy (2.15) for any $\left(\vec{W}_{\ell}, \vec{W}_{r}\right) \in \mathfrak{G}^{h}$. We also stress that any discontinuity of $\mathcal{R S}_{q_{0}}^{h}\left[\vec{W}_{\ell}, \vec{W}_{r}\right]$ satisfies the RankineHugoniot jump condition except the non-classical shocks along $x=0$. In fact, the latter discontinuities are defined to be stationary but, as the curve $w=v+p\left(q_{0} / v\right)$ is discretized over a grid, it happens generically that $q\left(\hat{W}^{h}\left(q_{0}, w_{\ell}\right)\right) \neq q\left(\check{W}^{h}\left(q_{0}, w_{\ell}\right)\right)$. Moreover, in general $\mathcal{R S}_{q_{0}}^{h}\left[\vec{W}_{\ell}, \vec{W}_{r}\right]$ does not satisfy (5.5c) because by (5.2b) it may happen that $\check{W}^{h}\left(q_{0}, w_{\ell}\right) \in \mathcal{C}^{h}\left(q_{0}\right)$.

\subsection{A priori estimates}

In this section we prove the main a priori estimates on the sequence of approximate solutions $\left(W^{h}\right)_{h}$. First, in Proposition 6 we prove that $\vec{W}^{h}(t)=\left(v^{h}(t), w^{h}(t)\right)^{T}$ takes values in $\mathcal{W}^{h}$ and we estimate $\operatorname{TV}\left(\vec{W}^{h}(t)\right)$ uniformly in $h$ and $t$. Then, in Proposition 7 we estimate the entropy corresponding to $\vec{W}^{h}$ as $h$ goes to infinity.

Since $\operatorname{TV}\left(w^{h}(t)\right)=\operatorname{TV}\left(w_{0}^{h}\right)$, it is sufficient estimate $\operatorname{TV}\left(v^{h}(t)\right)$. To this aim we introduce the Temple type functional

$$
\mathcal{B}(t) \doteq \mathrm{TV}\left(v^{h}(t)\right)+3 \mathrm{TV}\left(J^{h}\left(q_{0}, w^{h}(t)\right) ; \mathbb{R}_{-}\right)+2 \gamma(t)
$$

where $J^{h}$ is defined in (5.1) and

$$
\gamma(t) \doteq\left\{\begin{array}{lr}
0 & \text { if } W^{h}(t) \text { performs a non-classical shock } \\
\max \left\{J^{h}\left(q_{0}, w^{h}\left(t, 0^{-}\right)\right), J^{h}\left(q_{0}, w^{h}\left(t, 0^{+}\right)\right)\right\} & \text {otherwise. }
\end{array}\right.
$$


Proposition 6 For any fixed $h \in \mathbb{N}, \vec{W}_{0}^{h} \in \mathbf{P C}\left(\mathbb{R} ; \mathcal{W}^{h}\right)$, we have that:

1. $\vec{W}^{h}(t) \in \mathbf{P C}\left(\mathbb{R} ; \mathcal{W}^{h}\right)$ for all $t>0$;

2. the map $t \mapsto \mathcal{B}(t)$ is non increasing and it decreases by at least $\varepsilon^{h}$ each time the number of waves increases.

Proof For any $\bar{t}>0$ sufficiently small we know that the function $\vec{W}^{h}(\bar{t})$ is piecewise constant with jumps along a finite number of polygonal lines. If at time $\bar{t}$ either an interaction between waves takes place or a wave reaches $x=0$, then some of the waves may change speed and strength, while some new ones may be created, according to the solution of the Riemann problems at each of the points of discontinuity of $\vec{W}^{h}(\bar{t})$ and at $x=0$. Conventionally, we assume that the approximate solutions are left continuous in time, i.e. $\vec{W}^{h}(\bar{t})=\vec{W}^{h}(\bar{t}-)$. Then also $\mathcal{B}$ is left continuous in time and, for any $t$ in a sufficiently small left neighbourhood of $\bar{t}$, we can write

$$
\vec{W}^{h}(t, x)=\sum_{i \in \mathcal{J}^{h}} \vec{W}_{i+\frac{1}{2}}^{h} \chi_{\left[s_{i}^{h}(t), s_{i+1}^{h}(t)[\right.}(x),
$$

where $\mathcal{J}^{h} \subset \mathbb{Z}, \vec{W}_{i+1 / 2}^{h} \doteq\left(v_{i+1 / 2}^{h}, w_{i+1 / 2}^{h}\right)^{T} \in \mathcal{W}^{h}, s_{i-1}^{h}(t)<s_{i}^{h}(t), s_{0}^{h} \equiv 0$ and

$$
s_{i}^{h}(t) \doteq x_{i}^{h}+\sigma\left(\vec{W}_{i-\frac{1}{2}}^{h}, \vec{W}_{i+\frac{1}{2}}^{h}\right)(t-\bar{t}), \quad \vec{W}_{i-\frac{1}{2}}^{h} \neq \vec{W}_{i+\frac{1}{2}}^{h} \quad \text { for any } i \neq 0 .
$$

To prove that the approximate solution is well defined and keeps the form (5.6) we have to bound a priori the number of waves. To this aim we prove that the map $t \mapsto \mathcal{B}(t)$ is non-increasing and it decreases by at least $\varepsilon^{h}$ each time the number of waves increases. If at time $\bar{t}$ no interaction occurs, then $\Delta \mathcal{B} \doteq \mathcal{B}(\bar{t}+)-\mathcal{B}(\bar{t}-)=0$ and the number of waves does not change. Assume that at time $\bar{t}$ an interaction occurs. We have to distinguish the following two main cases:

(A) an interaction between waves takes place away from $x=0$;

(B) a wave reaches $x=0$.

For notational convenience we will write $q_{i}$ for $q\left(\vec{W}_{i}^{h}\right), \hat{W}_{i}$ for $\hat{W}^{h}\left(q_{0}, \vec{W}_{i}^{h}\right), \check{W}_{i}$ for $\check{W}^{h}\left(q_{0}, \vec{W}_{i}^{h}\right), \hat{v}_{i}$ for $\hat{v}^{h}\left(q_{0}, w_{i}^{h}\right)$, $\check{v}_{i}$ for $\check{v}^{h}\left(q_{0}, w_{i}^{h}\right)$ and $J_{i}$ for $J^{h}\left(q_{0}, w_{i}^{h}\right)$. Moreover, we omit the dependence on $h$ and write, for instance, $\varepsilon$ instead of $\varepsilon^{h}$ and $\tilde{w}$ instead of $\tilde{w}^{h}\left(q_{0}\right)$.

Let us consider the case (A). In this case $\Delta \mathcal{B}=\operatorname{TV}\left(v^{h}(\bar{t}+)\right)-\mathrm{TV}\left(v^{h}(\bar{t}-)\right)$. If the interaction does not involve vacuum states, then it is sufficient to apply the standard theory to obtain that the number of waves does not increase after the interaction and $\Delta \mathcal{B} \leq 0$, see [29]. The result follows from the fact that ARZ away from the vacuum is a Temple system. It remains to consider the cases involving vacuum states. For simplicity we describe in detail the interaction between two waves, one connecting $\vec{W}_{\ell}$ to $\vec{W}_{m}$ and the other connecting $\vec{W}_{m}$ to $\vec{W}_{r}$.

(A.1) If $\vec{W}_{\ell} \in \mathcal{W}_{0}$, then the speed of propagation of the first wave is $v_{m}$. Therefore the second wave cannot be a contact discontinuity, otherwise it would have the same speed of propagation. For the same reason $\vec{W}_{r}$ cannot belong to $\mathcal{W}_{0}$. Therefore the second wave has to be a shock or a rarefaction with $w_{r}=w_{m}$ and $\vec{W}_{r} \in \mathcal{W}_{0}^{c}$. The solution of the Riemann problem associated to the interaction is a discontinuity from $\vec{W}_{\ell}$ to $\vec{W}_{r}$ travelling with speed of propagation $v_{r}$. Therefore the number of waves decreases and

$$
\Delta \mathcal{B}=\left|v_{\ell}-v_{r}\right|-\left[\left|v_{\ell}-v_{m}\right|+\left|v_{m}-v_{r}\right|\right] \leq 0 .
$$

(A.2) If $\vec{W}_{m} \in \mathcal{W}_{0}$, then by (2.15) we have that $\vec{W}_{\ell}, \vec{W}_{r} \in \mathcal{W}_{0}^{c}$. Moreover $\vec{W}_{\ell}=\left(w_{m}-\varepsilon, w_{m}\right)^{T}$ and the speed of propagation of the first wave is $\left[w_{m}-\varepsilon\right]$. The second wave travels with speed of propagation $v_{r}$ and therefore it has to be $v_{r}<w_{m}-\varepsilon$. The solution of the Riemann problem associated to the interaction is a shock from $\vec{W}_{\ell}$ to $\vec{W}_{m}^{\prime}=\left(v_{r}, w_{m}\right)$ followed by a possibly null contact discontinuity from $\vec{W}_{m}^{\prime}$ to $\vec{W}_{r}$. Therefore the number of waves does not increase and

$$
\Delta \mathcal{B}=\left[\left(w_{m}-\varepsilon\right)-v_{r}\right]-\left[\varepsilon+\left(w_{m}-v_{r}\right)\right]=-2 \varepsilon \leq 0 .
$$

(A.3) If $\vec{W}_{r} \in \mathcal{W}_{0}$, then no interaction is possible. Indeed, in this case $\vec{W}_{m}=\left(w_{r}-\varepsilon, w_{r}\right) \in \mathcal{W}_{0}^{c}$ and the speed of propagation of the second wave is $\left[w_{r}-\varepsilon\right]$. On the other hand, the speed of propagation of the first wave is not greater than $\left[w_{r}-\varepsilon\right]$ for any $\vec{W}_{\ell}$.

(A.4) Assume finally that $\vec{W}_{\ell}, \vec{W}_{m}, \vec{W}_{r} \in \mathcal{W}_{0}^{c}$. If a vacuum state appears after the interaction, then it has to be $v_{r} \geq w_{\ell}$. The intersection of the set of all states that can be connected on the right of $\vec{W}_{\ell}$ with that one of all states that can be connected on the left of $\vec{W}_{r}$ is not empty if and only if $v_{r}=w_{\ell}=v_{\ell}+\varepsilon$. In this case the intersection selects $\vec{W}_{m} \doteq\left(v_{\ell}, w_{r}\right)^{T}$, the number of waves does not increase and clearly $\Delta \mathcal{B}=0$.

Let us consider now the case (B) with a detailed study of all possible interactions. For simplicity, we consider only interactions involving exactly two waves or a single wave reaching $x=0$.

(Р) We start with the cases in which the interaction involves a non-classical shock, namely $w_{-1 / 2}=w_{1 / 2} \geq \tilde{w}$, $v_{-1 / 2}=\hat{v}_{1 / 2}$ and $v_{1 / 2}=\check{v}_{1 / 2}$. Obviously $\gamma(\bar{t}-)=0$. Observe that no rarefaction wave can reach the non-classical shock. 
(B.1) If a contact discontinuity reaches the non-classical shock (from the left), then $v_{-3 / 2}=\hat{v}_{1 / 2}>0$ and $w_{-3 / 2} \neq w_{1 / 2}$. For later use, introduce $\vec{W}_{m} \doteq\left(\min \left\{\check{v}_{1 / 2}, w_{-3 / 2}\right\}, w_{-3 / 2}\right)^{T}$.

(B.1a) If $w_{-3 / 2}>w_{1 / 2}$, then $\mathcal{R S}_{q_{0}}^{h}\left[\vec{W}_{-3 / 2}, \vec{W}_{1 / 2}\right]$ performs a possible null shock from $\vec{W}_{-3 / 2}$ to $\hat{W}_{-3 / 2}$, followed by non-classical shock from $\hat{W}_{-3 / 2}$ to $\check{W}_{-3 / 2}$, followed by a shock from $\check{W}_{-3 / 2}$ to $\vec{W}_{m}$ and a contact discontinuity from $\vec{W}_{m}$ to $\vec{W}_{1 / 2}$. The number of waves of $\vec{W}^{h}$ therefore increases and by (5.2e)

$$
\Delta \mathcal{B}=2\left[J_{-3 / 2}-J_{1 / 2}\right]-3\left[J_{-3 / 2}-J_{1 / 2}\right]=-\left[J_{-3 / 2}-J_{1 / 2}\right] \leq-\varepsilon
$$

(B.1b) If $\tilde{w} \leq w_{-3 / 2}<w_{1 / 2}$ and $\check{v}_{1 / 2}<w_{-3 / 2}$, then $\mathcal{R S}_{q_{0}}^{h}\left[\vec{W}_{-3 / 2}, \vec{W}_{1 / 2}\right]$ performs a possible null discretized rarefaction from $\vec{W}_{-3 / 2}$ to $\hat{W}_{-3 / 2}$, followed by non-classical shock from $\hat{W}_{-3 / 2}$ to $\breve{W}_{-3 / 2}$, followed by a discretized rarefaction from $\check{W}_{-3 / 2}$ to $\vec{W}_{m} \in \mathcal{W}_{0}^{c}$ and a contact discontinuity from $\vec{W}_{m}$ to $\vec{W}_{1 / 2}$. The number of waves of $\vec{W}^{h}$ therefore increases and by $(5.2 \mathrm{e})$

$$
\Delta \mathcal{B}=-3\left[J_{1 / 2}-J_{-3 / 2}\right]<-\varepsilon
$$

(B.1c) If $\tilde{w} \leq w_{-3 / 2}<w_{1 / 2}$ and $w_{-3 / 2} \leq \check{v}_{1 / 2}$, then $\mathcal{R} \mathcal{S}_{q_{0}}^{h}\left[\vec{W}_{-3 / 2}, \vec{W}_{1 / 2}\right]$ performs a possible null discretized rarefaction from $\vec{W}_{-3 / 2}$ to $\hat{W}_{-3 / 2}$, followed by non-classical shock from $\hat{W}_{-3 / 2}$ to $\check{W}_{-3 / 2}$, followed by a discretized rarefaction from $\check{W}_{-3 / 2}$ to $\vec{W}_{m} \in \mathcal{W}_{0}$ and a discontinuity from $\vec{W}_{m}$ to $\vec{W}_{1 / 2}$. The number of waves of $\vec{W}^{h}$ therefore increases and by $(5.2 \mathrm{e})$

$$
\Delta \mathcal{B}=-3\left[J_{1 / 2}-J_{-3 / 2}\right]<-\varepsilon
$$

(B.1d) If $\check{v}_{1 / 2}<w_{-3 / 2}<\tilde{w}$, then $J_{-3 / 2}=0$ and $\mathcal{R S}_{q_{0}}^{h}\left[\vec{W}_{-3 / 2}, \vec{W}_{1 / 2}\right]$ performs a discretized rarefaction from $\vec{W}_{-3 / 2}$ to $\vec{W}_{m} \in \mathcal{W}_{0}^{c}$ and a contact discontinuity from $\vec{W}_{m}$ to $\vec{W}_{1 / 2}$. The number of waves of $\vec{W}^{h}$ therefore increases and by $(5.2 \mathrm{~d})$

$$
\Delta \mathcal{B}=-3 J_{1 / 2}<-\varepsilon
$$

(B.1e) If $w_{-3 / 2}<\tilde{w}$ and $w_{-3 / 2} \leq \check{v}_{1 / 2}$, then $J_{-3 / 2}=0$ and $\mathcal{R S}_{q_{0}}^{h}\left[\vec{W}_{-3 / 2}, \vec{W}_{1 / 2}\right]$ performs a discretized rarefaction from $\vec{W}_{-3 / 2}$ to $\vec{W}_{m} \in \mathcal{W}_{0}$ and a discontinuity from $\vec{W}_{m}$ to $\vec{W}_{1 / 2}$. The number of waves of $\vec{W}^{h}$ therefore increases and by $(5.2 \mathrm{~d})$

$$
\Delta \mathcal{B}=-3 J_{1 / 2}<-\varepsilon .
$$

(B.2) If a shock reaches the non-classical shock, then $w_{-3 / 2}=w_{ \pm 1 / 2}, v_{-3 / 2} \geq \check{v}_{1 / 2}$ and the result of the interaction is a possible null single shock and

$$
\Delta \mathcal{B}=-2 J_{1 / 2}+2 J_{1 / 2}=0 .
$$

$(\diamond)$ Assume now that $\vec{W}_{-1 / 2}=\vec{W}_{1 / 2}$. Obviously $\gamma(\bar{t}-)=J_{1 / 2}$.

(B.3) If a contact discontinuity reaches $x=0$ (from the left), then $v_{-3 / 2}=v_{-1 / 2}=v_{1 / 2}>0$ and $w_{-3 / 2} \neq w_{1 / 2}$.

(B.3a) If $\vec{W}_{-3 / 2} \notin \mathcal{C}^{h}$, then $\mathcal{R S}_{q_{0}}^{h}\left[\vec{W}_{-3 / 2}, \vec{W}_{1 / 2}\right]$ performs a single contact discontinuity and by $(5.2 \mathrm{e})$

$$
\Delta \mathcal{B}=-3\left|J_{-3 / 2}-J_{1 / 2}\right|+2\left[J_{-3 / 2}-J_{1 / 2}\right] \leq 0 .
$$

(B.3b) If $\vec{W}_{-3 / 2} \in \mathcal{C}^{h}$, then $w_{-3 / 2}>w_{1 / 2}, w_{-3 / 2} \geq \tilde{w}, \mathcal{R S}_{q_{0}}^{h}\left[\vec{W}_{-3 / 2}, \vec{W}_{1 / 2}\right]$ performs a shock from $\vec{W}_{-3 / 2}$ to $\hat{W}_{-3 / 2}$, followed by a non-classical shock from $\hat{W}_{-3 / 2}$ to $\check{W}_{-3 / 2}$, a shock from $\check{W}_{-3 / 2}$ to $\vec{W}_{-3 / 2}$ and a contact discontinuity from $\vec{W}_{-3 / 2}$ to $\vec{W}_{1 / 2}$. The number of waves increases and by (5.2e)

$$
\Delta \mathcal{B}=2 J_{-3 / 2}-3\left[J_{-3 / 2}-J_{1 / 2}\right]-2 J_{1 / 2}=-\left[J_{-3 / 2}-J_{1 / 2}\right] \leq-\varepsilon .
$$

(B.4) If a shock reaches $x=0$, then the result of the interaction is a single shock, the number of waves does not change and $\Delta \mathcal{B}=0$.

(B.5) If a rarefaction reaches $x=0$, then we have to distinguish two possible cases.

(B.5a) If after the interaction the solution does not perform a non-classical shock, then it performs a single rarefaction, the number of waves does not change and $\Delta \mathcal{B}=0$.

(B.5b) The only case in which after the interaction the solution involves a non-classical shock is when $\vec{W}_{ \pm 1 / 2}=$ $\check{W}_{1 / 2}$ and the incoming rarefaction has positive speed of propagation. Then the solution performs a shock from $\vec{W}_{-3 / 2}$ to $\hat{W}_{1 / 2}$, followed by a non-classical shock from $\hat{W}_{-3 / 2}$ to $\check{W}_{1 / 2}$. The number of waves increases and

$$
\Delta \mathcal{B}=2\left[J_{1 / 2}-\varepsilon\right]-2 J_{1 / 2}=-2 \varepsilon<-\varepsilon .
$$


(\$) Assume now to have a stationary shock at $x=0$, namely $v_{-1 / 2}>v_{1 / 2}, w_{-1 / 2}=w_{1 / 2}$ and $q\left(\vec{W}_{-1 / 2}\right)=$ $q\left(\vec{W}_{1 / 2}\right)$. Obviously $\gamma(\bar{t}-)=J_{1 / 2}$.

(B.6) If a contact discontinuity reaches $x=0$ (from the left), then $v_{-3 / 2}=v_{-1 / 2}, w_{-3 / 2} \neq w_{-1 / 2}$ and we have to distinguish two possible cases.

(B.6a) If the solution after the interaction does not perform any non-classical shock, then the number of waves does not change and

$$
\Delta \mathcal{B}=-3\left|J_{-3 / 2}-J_{1 / 2}\right|+2\left[J_{-3 / 2}-J_{1 / 2}\right] \leq 0 .
$$

(B.6b) If the solution after the interaction performs a non-classical shock, then the number of waves increases and by $(5.2 \mathrm{e})$

$$
\Delta \mathcal{B}=2 J_{-3 / 2}-3\left[J_{-3 / 2}-J_{1 / 2}\right]-2 J_{1 / 2}=-\left[J_{-3 / 2}-J_{1 / 2}\right] \leq-\varepsilon .
$$

(B.7) If a shock reaches $x=0$, then the result of the interaction is a single shock and $\Delta \mathcal{B}=0$.

(B.8) If a rarefaction reaches $x=0$, then the result of the interaction is a single shock, the number of waves decreases and $\Delta \mathcal{B}=-2 \varepsilon \leq 0$.

(\$) Assume now to have a stationary rarefaction, namely $v_{-1 / 2}=v_{1 / 2}-\varepsilon, w_{-1 / 2}=w_{1 / 2}<\tilde{w}$. Obviously $\gamma(\bar{t}-)=J_{1 / 2}=0$.

(B.9) If a contact discontinuity reaches $x=0$ (from the left), then we have to distinguish two cases.

(B.9a) If $\mathcal{R S}_{q_{0}}^{h}\left[\vec{W}_{-3 / 2}, \vec{W}_{1 / 2}\right]$ does not performs any non-classical shock, then the number of waves does not change and $\Delta \mathcal{B}=-3 J_{-3 / 2}+2 J_{-3 / 2} \leq 0$.

(B.9b) If $\mathcal{R S}_{q_{0}}^{h}\left[\vec{W}_{-3 / 2}, \vec{W}_{1 / 2}\right]$ performs a non-classical shock, then the number of waves increases and by (5.2d)

$$
\Delta \mathcal{B}=2\left[J_{-3 / 2}-\varepsilon\right]-3 J_{-3 / 2}=-\left[J_{-3 / 2}+2 \varepsilon\right]<-\varepsilon .
$$

(B.10) If a shock reaches $x=0$, then the result of the interaction is a single shock and $\Delta \mathcal{B}=-2\left[v_{1 / 2}-v_{-1 / 2}\right]=$ $-2 \varepsilon \leq 0$.

( $\star$ ) Assume to have a stationary contact discontinuity, namely $v_{-1 / 2}=v_{1 / 2}=0, w_{-1 / 2} \neq w_{1 / 2}$. Obviously $\gamma(\bar{t}-)=0$.

(B.11) If a rarefaction reaches $x=0$ (from the right), then the number of waves does not change and $\Delta \mathcal{B}=$ $2\left[J_{-1 / 2}-\max \left\{J_{-1 / 2}, J_{1 / 2}\right\}\right] \leq 0$.

This concludes the proof.

Proposition $\mathbf{7}$ For any non-negative test function $\phi \in \mathbf{C}_{\mathbf{c}}^{\infty}(] 0,+\infty[\times \mathbb{R} ; \mathbb{R})$ and $\left.k \in\right] 0,+\infty[$

$$
\begin{aligned}
\liminf _{h \rightarrow+\infty} & {\left[\int_{\mathbb{R}_{+}} \int_{\mathbb{R}}\left[\mathcal{E}_{k}\left(\vec{W}^{h}\right) \phi_{t}+\mathcal{Q}_{k}\left(\vec{W}^{h}\right) \phi_{x}\right] \mathrm{d} x \mathrm{~d} t\right.} \\
& \left.+\int_{\mathbb{R}_{+}} q\left(\vec{W}^{h}\left(t, 0^{+}\right)\right)\left[\frac{k}{q_{0}}-\frac{1}{p^{-1}\left(\left[w^{h}(t, 0)-k\right]^{+}\right)}\right]^{+} \phi(t, 0) \mathrm{d} t\right] \geq 0 .
\end{aligned}
$$

Proof In the following we will denote $\rho_{*}^{h}$ for $\rho\left(\vec{W}_{*}^{h}\right)$ and $q_{*}^{h}$ for $q\left(\vec{W}_{*}^{h}\right)$. Choose $T>0$ so that $\phi$ vanishes outside the strip $] 0, T[\times \mathbb{R}$. Using the Green-Gauss formula, the double integral above can be computed as

$$
\int_{0}^{T} \sum_{i \in \mathcal{J}^{h}}\left[\dot{s}_{i}^{h}(t) \Delta \mathcal{E}_{k}^{i}(t)-\Delta \mathcal{Q}_{k}^{i}(t)\right] \phi\left(t, s_{i}^{h}(t)\right) \mathrm{d} t
$$

where, with the same notation introduced in (5.6),

$$
\Delta \mathcal{E}_{k}^{i}(t) \doteq \mathcal{E}_{k}\left(\vec{W}_{i+\frac{1}{2}}^{h}\right)-\mathcal{E}_{k}\left(\vec{W}_{i-\frac{1}{2}}^{h}\right), \quad \quad \Delta \mathcal{Q}_{k}^{i}(t) \doteq \mathcal{Q}_{k}\left(\vec{W}_{i+\frac{1}{2}}^{h}\right)-\mathcal{Q}_{k}\left(\vec{W}_{i-\frac{1}{2}}^{h}\right) .
$$

To estimate the above integral, we have to consider separately the cases in which the $i$ th discontinuity is a nonclassical shock, a shock, a discretized rarefaction or a contact discontinuity.

- If $\vec{W}^{h}(t)$ performs a non-classical shock, then $w_{-1 / 2}^{h}=w_{1 / 2}^{h}$ and

$$
-\Delta \mathcal{Q}_{k}^{0}(t)= \begin{cases}0 & \text { if } \check{v}^{h}\left(q_{0}, w_{\frac{1}{2}}^{h}\right) \leq k, \\ \frac{q\left(\check{W}^{h}\left(q_{0}, w_{\frac{1}{2}}^{h}\right)\right)}{p^{-1}\left(w_{\frac{1}{2}}^{h}-k\right)}-k & \text { if } \hat{v}^{h}\left(q_{0}, w_{\frac{1}{2}}^{h}\right) \leq k<\check{v}^{h}\left(q_{0}, w_{\frac{1}{2}}^{h}\right), \\ \frac{q\left(\check{W}^{h}\left(q_{0}, w_{\frac{1}{2}}^{h}\right)\right)-q\left(\hat{W}^{h}\left(q_{0}, w_{\frac{1}{2}}^{h}\right)\right)}{p^{-1}\left(w_{\frac{1}{2}}^{h}-k\right)} & \text { if } k<\hat{v}^{h}\left(q_{0}, w_{\frac{1}{2}}^{h}\right) .\end{cases}
$$


Therefore, by $(5.2 \mathrm{~b})$ we know that $-\Delta \mathcal{Q}_{k}^{0}(t)<0$ if and only if

$$
\hat{v}^{h}\left(q_{0}, w_{\frac{1}{2}}^{h}\right) \leq k<\check{v}^{h}\left(q_{0}, w_{\frac{1}{2}}^{h}\right) \quad \text { and } \quad q\left(\check{W}^{h}\left(q_{0}, w_{\frac{1}{2}}^{h}\right)\right)<p^{-1}\left(w_{\frac{1}{2}}^{h}-k\right) k .
$$

However, in this case by $(5.2 \mathrm{~b})$ we have that $p^{-1}\left(w_{1 / 2}^{h}-k\right) k>q_{0}$ and therefore

$$
\begin{aligned}
& -\Delta \mathcal{Q}_{k}^{0}(t)+q\left(\vec{W}^{h}\left(t, 0^{+}\right)\right)\left[\frac{k}{q_{0}}-\frac{1}{p^{-1}\left(\left[w_{\frac{1}{2}}^{h}-k\right]^{+}\right)}\right]^{+} \\
= & {\left[\frac{q\left(\check{W}^{h}\left(q_{0}, w_{\frac{1}{2}}^{h}\right)\right)}{p^{-1}\left(w_{\frac{1}{2}}^{h}-k\right)}-k\right]+q\left(\check{W}^{h}\left(q_{0}, w_{\frac{1}{2}}^{h}\right)\right)\left[\frac{k}{q_{0}}-\frac{1}{p^{-1}\left(w_{\frac{1}{2}}^{h}-k\right)}\right]=\left[\frac{q\left(\check{W}^{h}\left(q_{0}, w_{\frac{1}{2}}^{h}\right)\right)}{q_{0}}-1\right] k \geq 0 . }
\end{aligned}
$$

- If the $i$ th discontinuity is of the first family, then $v_{i-\frac{1}{2}}^{h} \neq v_{i+\frac{1}{2}}^{h}, w_{i-\frac{1}{2}}^{h}=w_{i+\frac{1}{2}}^{h}=w$. For any $k \neq w$

$$
\begin{aligned}
\dot{s}_{i}^{h}(t) \Delta \mathcal{E}_{k}^{i}(t)-\Delta \mathcal{Q}_{k}^{i}(t)=\frac{q_{i+\frac{1}{2}}^{h}-q_{i-\frac{1}{2}}^{h}}{\rho_{i+\frac{1}{2}}^{h}-\rho_{i-\frac{1}{2}}} \times \\
\times\left[\operatorname{sign}^{+}\left(v_{i+\frac{1}{2}}^{h}-k\right)\left[1-\frac{\rho_{i+\frac{1}{2}}^{h}}{p^{-1}(w-k)}\right]-\operatorname{sign}^{+}\left(v_{i-\frac{1}{2}}^{h}-k\right)\left[1-\frac{\rho_{i-\frac{1}{2}}^{h}}{p^{-1}(w-k)}\right]\right] \\
-\left[\operatorname{sign}^{+}\left(v_{i+\frac{1}{2}}^{h}-k\right)\left[k-\frac{q_{i+\frac{1}{2}}^{h}}{p^{-1}(w-k)}\right]-\operatorname{sign}^{+}\left(v_{i-\frac{1}{2}}^{h}-k\right)\left[k-\frac{q_{i-\frac{1}{2}}^{h}}{p^{-1}(w-k)}\right]\right] \\
=\frac{\operatorname{sign}^{+}\left(v_{i+\frac{1}{2}}^{h}-k\right)}{p^{-1}(w-k)}\left[q_{i+\frac{1}{2}}^{h}+\frac{q_{i+\frac{1}{2}}^{h}-q_{i-\frac{1}{2}}^{h}}{\rho_{i+\frac{1}{2}}^{h}-\rho_{i-\frac{1}{2}}}\left[p^{-1}(w-k)-\rho_{i+\frac{1}{2}}^{h}\right]-p^{-1}(w-k) k\right] \\
-\frac{\operatorname{sign}^{+}\left(v_{i-\frac{1}{2}}^{h}-k\right)}{p^{-1}(w-k)}\left[q_{i-\frac{1}{2}}^{h}+\frac{q_{i+\frac{1}{2}}^{h}-q_{i-\frac{1}{2}}^{h}}{\rho_{i+\frac{1}{2}}^{h}-\rho_{i-\frac{1}{2}}}\left[p^{-1}(w-k)-\rho_{i-\frac{1}{2}}^{h}\right]-p^{-1}(w-k) k\right]
\end{aligned}
$$

and therefore

○ if $k \leq \min \left\{v_{i-\frac{1}{2}}^{h}, v_{i+\frac{1}{2}}^{h}\right\}$ or $k \geq \max \left\{v_{i-\frac{1}{2}}^{h}, v_{i+\frac{1}{2}}^{h}\right\}$, then we have

$$
\dot{s}_{i}^{h}(t) \Delta \mathcal{E}_{k}^{i}(t)-\Delta \mathcal{Q}_{k}^{i}(t)=0 ;
$$

○ if $v_{i+\frac{1}{2}}^{h}<v_{i-\frac{1}{2}}^{h}$, then for all $\left.k \in\right] v_{i+\frac{1}{2}}^{h}, v_{i-\frac{1}{2}}^{h}[$ we have

$$
\dot{s}_{i}^{h}(t) \Delta \mathcal{E}_{k}^{i}(t)-\Delta \mathcal{Q}_{k}^{i}(t)=-\frac{1}{p^{-1}(w-k)}\left[q_{i-\frac{1}{2}}^{h}+\frac{q_{i+\frac{1}{2}}^{h}-q_{i-\frac{1}{2}}^{h}}{\rho_{i+\frac{1}{2}}^{h}-\rho_{i-\frac{1}{2}}}\left[p^{-1}(w-k)-\rho_{i-\frac{1}{2}}^{h}\right]-p^{-1}(w-k) k\right] \geq 0
$$

because $\rho \mapsto[w-p(\rho)] \rho$ is concave;

○ if $v_{i+\frac{1}{2}}^{h}=v_{i-\frac{1}{2}}^{h}+\varepsilon^{h}$, then for all $\left.k \in\right] v_{i-\frac{1}{2}}^{h}, v_{i+\frac{1}{2}}^{h}[$ we have

$$
\begin{aligned}
\dot{s}_{i}^{h}(t) \Delta \mathcal{E}_{k}^{i}(t)-\Delta \mathcal{Q}_{k}^{i}(t) & =\frac{1}{p^{-1}(w-k)}\left[q_{i+\frac{1}{2}}^{h}+\frac{q_{i+\frac{1}{2}}^{h}-q_{i-\frac{1}{2}}^{h}}{\rho_{i+\frac{1}{2}}^{h}-\rho_{i-\frac{1}{2}}}\left[p^{-1}(w-k)-\rho_{i+\frac{1}{2}}^{h}\right]-p^{-1}(w-k) k\right] \\
& =-\left[1-\frac{\rho_{i+\frac{1}{2}}^{h}}{p^{-1}(w-k)}\right]\left[\frac{p^{-1}(w-k) k-q_{i+\frac{1}{2}}^{h}}{p^{-1}(w-k)-\rho_{i+\frac{1}{2}}^{h}}-\frac{q_{i+\frac{1}{2}}^{h}-q_{i-\frac{1}{2}}^{h}}{\rho_{i+\frac{1}{2}}^{h}-\rho_{i-\frac{1}{2}}}\right] \\
& \geq-\left[1-\frac{\rho_{i+\frac{1}{2}}^{h}}{\rho_{i-\frac{1}{2}}^{h}}\right]\left[\left[v_{i+\frac{1}{2}}^{h}-\rho_{i+\frac{1}{2}}^{h} p^{\prime}\left(\rho_{i+\frac{1}{2}}^{h}\right)\right]-\left[v_{i-\frac{1}{2}}^{h}-\rho_{i-\frac{1}{2}}^{h} p^{\prime}\left(\rho_{i-\frac{1}{2}}^{h}\right)\right]\right] \\
& \geq-\max _{\rho \in\left[0, p^{-1}\left(V_{0}\right)\right]}\left[2+\frac{\rho p^{\prime \prime}(\rho)}{p^{\prime}(\rho)}\right]\left[v_{i+\frac{1}{2}}^{h}-v_{i-\frac{1}{2}}^{h}\right],
\end{aligned}
$$


because for any $0 \leq a<b \leq w$

$$
\begin{aligned}
& b-\rho(b, w) p^{\prime}(\rho(b, w)) \leq \frac{q(a, w)-q(b, w)}{\rho(a, w)-\rho(b, w)} \leq a-\rho(a, w) p^{\prime}(\rho(a, w)), \\
& \partial_{v}\left[v-p^{-1}(w-v) p^{\prime}\left(p^{-1}(w-v)\right)\right]=2+\frac{p^{-1}(w-v) p^{\prime \prime}\left(p^{-1}(w-v)\right)}{p^{\prime}\left(p^{-1}(w-v)\right)} .
\end{aligned}
$$

Observe that the above maximum is bounded uniformly due to assumption (2.2).

- If the $i$ th discontinuity is of the second family, then $v_{i-\frac{1}{2}}^{h}=v_{i+\frac{1}{2}}^{h}=v, w_{i-\frac{1}{2}}^{h} \neq w_{i+\frac{1}{2}}^{h}$. For any $k \geq v$ we have $\dot{s}_{i}^{h}(t) \Delta \mathcal{E}_{k}^{i}(t)-\Delta \mathcal{Q}_{k}^{i}(t)=0$. Otherwise, if $k<v$ then

$$
\dot{s}_{i}^{h}(t) \Delta \mathcal{E}_{k}^{i}(t)-\Delta \mathcal{Q}_{k}^{i}(t)=v\left[\frac{\rho_{i-\frac{1}{2}}^{h}}{p^{-1}\left(w_{i-\frac{1}{2}}^{h}-k\right)}-\frac{\rho_{i+\frac{1}{2}}^{h}}{p^{-1}\left(w_{i+\frac{1}{2}}^{h}-k\right)}\right]-\left[\frac{q_{i-\frac{1}{2}}^{h}}{p^{-1}\left(w_{i-\frac{1}{2}}^{h}-k\right)}-\frac{q_{i+\frac{1}{2}}^{h}}{p^{-1}\left(w_{i+\frac{1}{2}}^{h}-k\right)}\right]=0 .
$$

In conclusion we proved that

$$
\begin{aligned}
& \liminf _{h \rightarrow+\infty}\left\{\int_{0}^{T} \sum_{i \in \mathcal{J}^{h}}\left[\dot{s}_{i}^{h}(t) \Delta \mathcal{E}_{k}^{i}(t)-\Delta \mathcal{Q}_{k}^{i}(t)\right] \phi\left(t, s_{i}^{h}(t)\right) \mathrm{d} t+\int_{\mathbb{R}_{+}}\left[k-\frac{q_{0}}{p^{-1}\left(\left[w^{h}-k\right]^{+}\right)}\right]^{+} \phi(t, 0) \mathrm{d} t\right\} \\
\geq & \liminf _{h \rightarrow+\infty} \int_{0}^{T} \sum_{i \in \mathcal{R}^{h}(t)}\left[\dot{s}_{i}^{h}(t) \Delta \mathcal{E}_{k}^{i}(t)-\Delta \mathcal{Q}_{k}^{i}(t)\right] \phi\left(t, s_{i}^{h}(t)\right) \mathrm{d} t,
\end{aligned}
$$

where $\mathcal{R}^{h}(t)$ is the set of indexes corresponding to discretized rarefactions. Moreover, for all $i \in \mathcal{R}^{h}(t)$ we proved that

$$
\begin{array}{ll}
\dot{s}_{i}^{h}(t) \Delta \mathcal{E}_{k}^{i}(t)-\Delta \mathcal{Q}_{k}^{i}(t)=0 & \text { if } k \notin] v_{i-\frac{1}{2}}^{h}, v_{i+\frac{1}{2}}^{h}[ \\
-m\left[v_{i+\frac{1}{2}}^{h}-v_{i-\frac{1}{2}}^{h}\right] \leq \dot{s}_{i}^{h}(t) \Delta \mathcal{E}_{k}^{i}(t)-\Delta \mathcal{Q}_{k}^{i}(t) \leq 0 & \text { if } k \in] v_{i-\frac{1}{2}}^{h}, v_{i+\frac{1}{2}}^{h}[
\end{array}
$$

where $m \doteq \max _{\rho \in\left[0, p^{-1}\left(V_{0}\right)\right]}\left[2+\frac{\rho p^{\prime \prime}(\rho)}{p^{\prime}(\rho)}\right]$. By (5.10) we have that TV $\left(v^{h}(t)\right)$ is bounded by $C$ uniformly in $t$ and $h$,

$$
\mathrm{TV}\left(v^{h}(t)\right)=\sum_{i \in \mathcal{J}^{h}}\left|v_{i+\frac{1}{2}}^{h}-v_{i-\frac{1}{2}}^{h}\right| \leq C
$$

and therefore

$$
-M \leq \int_{0}^{T} \sum_{i \in \mathcal{R}^{h}(t)}\left[\dot{s}_{i}^{h}(t) \Delta \mathcal{E}_{k}^{i}(t)-\Delta \mathcal{Q}_{k}^{i}(t)\right] \phi\left(t, s_{i}^{h}(t)\right) \mathrm{d} t \leq 0,
$$

where $M \doteq m C T\|\phi\|_{\infty}$. Since this estimate is not enough to conclude, we have to improve it by considering simultaneously different values of $k$. We will show that for $h$ sufficiently large the above integral can be bounded from below by a negative term as close to zero as desired for a dense set of values of $k$ in $\left[0, V_{0}\right]$. More explicitly, for any fixed $n>0$, we want to prove that there exists a dense set of values of $k$ in $\left[0, V_{0}\right]$ such that

$$
\liminf _{h \rightarrow+\infty} \int_{0}^{T} \sum_{i \in \mathcal{R}^{h}(t)}\left[\dot{s}_{i}^{h}(t) \Delta \mathcal{E}_{k}^{i}(t)-\Delta \mathcal{Q}_{k}^{i}(t)\right] \phi\left(t, s_{i}^{h}(t)\right) \mathrm{d} t \geq-\frac{1}{n} .
$$

Fix $a, b \in\left[0, V_{0}\right]$ with $a<b$. We have to prove that there exists $\left.k \in\right] a, b[$ such that the above estimate holds true. Let $l=\left\lfloor\frac{2 M n}{b-a}\right\rfloor+1$ and introduce the set

$$
\left.\mathcal{K}_{n} \doteq \frac{2 \mathbb{N}+1}{l} \cap\right] a, b[
$$

Assume that $\mathfrak{h}_{n} \in \mathbb{N}$ is sufficiently large to have $\varepsilon^{\mathfrak{h}_{n}}<2 / l$ and take $h \geq \mathfrak{h}_{n}$. Then, for any $i \in \mathcal{R}^{h}(t)$ we have that

$$
v_{i+\frac{1}{2}}^{h}-v_{i-\frac{1}{2}}^{h}=\varepsilon^{h}<\frac{2}{l}=\min _{\substack{k, k^{\prime} \in \mathcal{K}_{n} \\ k \neq k^{\prime}}}\left|k-k^{\prime}\right|,
$$

hence $\left.\mathcal{K}_{n} \cap\right] v_{i-\frac{1}{2}}^{h}, v_{i+\frac{1}{2}}^{h}[$ has at most one element. Therefore the sum

$$
\sum_{k \in \mathcal{K}_{n}}\left[\dot{s}_{i}^{h}(t) \Delta \mathcal{E}_{k}^{i}(t)-\Delta \mathcal{Q}_{k}^{i}(t)\right]
$$


has at most one non-zero element, moreover by (5.8) it is bounded from below by $-m\left[v_{i+\frac{1}{2}}^{h}-v_{i-\frac{1}{2}}^{h}\right]$. Therefore by (5.9) we find

$$
\sum_{i \in \mathcal{R}^{h}(t)} \sum_{k \in \mathcal{K}_{n}}\left[\dot{s}_{i}^{h}(t) \Delta \mathcal{E}_{k}^{i}(t)-\Delta \mathcal{Q}_{k}^{i}(t)\right] \geq-m C .
$$

By exchanging sums with the integral in $t \in[0, T]$, we find

$$
\sum_{k \in \mathcal{K}_{n}} \int_{0}^{T} \sum_{i \in \mathcal{R}^{h}(t)}\left[\dot{s}_{i}^{h}(t) \Delta \mathcal{E}_{k}^{i}(t)-\Delta \mathcal{Q}_{k}^{i}(t)\right] \phi\left(t, s_{i}^{h}(t)\right) \mathrm{d} t \geq-M .
$$

Moreover, by construction we have that $\mathcal{K}_{n}$ is a non-empty set (it has at least $(M n)$ elements) with a finite number of elements (it has at most $\left(M n+\frac{b-a}{2}\right)$ elements) and therefore

$$
M n \max _{k \in \mathcal{K}_{n}}\left[\int_{0}^{T} \sum_{i \in \mathcal{R}^{h}(t)}\left[\dot{s}_{i}^{h}(t) \Delta \mathcal{E}_{k}^{i}(t)-\Delta \mathcal{Q}_{k}^{i}(t)\right] \phi\left(t, s_{i}^{h}(t)\right) \mathrm{d} t\right] \geq-M .
$$

In conclusion we proved that there exists $\left.k \in \mathcal{K}_{n} \subseteq\right] a, b\left[\right.$ such that the above estimate holds true for any $h \geq \mathfrak{h}_{n}$; therefore, since $\mathcal{K}_{n}$ has a finite number of elements, we have

$$
\liminf _{h \rightarrow+\infty} \int_{0}^{T} \sum_{i \in \mathcal{R}^{h}(t)}\left[\dot{s}_{i}^{h}(t) \Delta \mathcal{E}_{k}^{i}(t)-\Delta \mathcal{Q}_{k}^{i}(t)\right] \phi\left(t, s_{i}^{h}(t)\right) \mathrm{d} t \geq-\frac{1}{n} .
$$

As $a$ and $b$ are arbitrary, the above estimate holds true for a dense set of values of $k$ in $\left[0, V_{0}\right]$.

Actually, the above estimate holds for any $k$ in $\left[0, V_{0}\right]$ because the term in brackets in the above formula is continuous with respect to $k$. Finally, for the arbitrariness of $n$, we have that

$$
\liminf _{h \rightarrow+\infty} \int_{0}^{T} \sum_{i \in \mathcal{R}^{h}(t)}\left[\dot{s}_{i}^{h}(t) \Delta \mathcal{E}_{k}^{i}(t)-\Delta \mathcal{Q}_{k}^{i}(t)\right] \phi\left(t, s_{i}^{h}(t)\right) \mathrm{d} t=0
$$

and this concludes the proof.

\subsection{Convergence}

In this section we prove that $\left(\vec{W}^{h}\right)_{h}$ converges in $\mathbf{L}_{\text {loc }}^{\mathbf{1}}$ to $\vec{W}$ (along a subsequence) and that $\vec{W}$ is in fact a constrained entropy solution of (3.4) in the sense of Definition 5 .

For any $t>0$ we have that $\operatorname{TV}\left(w^{h}(t)\right)=\operatorname{TV}\left(w_{0}^{h}\right)$ and by Proposition 6 that

$$
\operatorname{TV}\left(\vec{W}^{h}(t)\right) \leq \mathcal{B}(t)+\operatorname{TV}\left(w^{h}(t)\right) \leq \mathcal{B}(0)+\operatorname{TV}\left(w_{0}^{h}\right) \leq C,
$$

where by (5.4)

$$
C \doteq \mathrm{TV}\left(\vec{W}_{0}\right)+3 \mathrm{TV}\left(J\left(q_{0}, w_{0}\right) ; \mathbb{R}_{-}\right)+2 V_{0}
$$

Moreover, observe that $\left\|\vec{W}^{h}(t)\right\|_{\infty} \leq V_{0}$ and

$$
\left\|\vec{W}^{h}(t)-\vec{W}^{h}(s)\right\|_{1} \leq L|t-s|
$$

with

$$
L \doteq C \max \left\{V_{0}, p^{-1}\left(V_{0}\right) p^{\prime}\left(p^{-1}\left(V_{0}\right)\right)\right\}
$$

Indeed, if no interaction occurs for times between $t$ and $s$, then

$$
\left\|\vec{W}^{h}(t)-\vec{W}^{h}(s)\right\|_{1} \leq \sum_{i \neq 0}\left\|(t-s) \dot{s}_{i}^{h}(t)\left(\vec{W}_{i-1 / 2}^{h}-\vec{W}_{i+1 / 2}^{h}\right)\right\| \leq L|t-s| .
$$

The case when one or more interactions take place for times between $t$ and $s$ is similar, because by the finite speed of propagation of the waves, the map $t \mapsto \vec{W}^{h}(t)$ is $\mathbf{L}^{\mathbf{1}}$-continuous across interaction times.

Thus, by applying Helly's Theorem in the form $\left[9\right.$, Theorem 2.4], there exists a function $\vec{W} \in \mathbf{L}_{\text {loc }}^{\mathbf{1}}\left(\mathbb{R}_{+} \times \mathbb{R} ;\left[0, V_{0}\right]^{2}\right)$ and a subsequence, still denoted $\left(\vec{W}^{h}\right)_{h}$, such that $\left(\vec{W}^{h}\right)_{h}$ converges to $\vec{W}$ in $\mathbf{L}_{\text {loc }}^{\mathbf{1}}\left(\mathbb{R}_{+} \times \mathbb{R} ;\left[0, V_{0}\right]^{2}\right)$ as $h$ goes to infinity. Moreover, $\vec{W}$ satisfies the estimates (3.11), namely for all $t, s \in \mathbb{R}_{+}$

$$
\operatorname{TV}(\vec{W}(t)) \leq C, \quad\|\vec{W}(t)-\vec{W}(s)\|_{1} \leq L|t-s|, \quad \quad\|\vec{W}(t)\|_{\infty} \leq V_{0}
$$


Proposition $8 \vec{W}$ is a constrained weak solution of (3.4) in the sense of Definition 5.

Proof First, we prove that $\vec{W}$ satisfies (2.7). The initial condition (3.4b) holds by (5.3), (5.11) and the $\mathbf{L}_{\text {loc }}^{\mathbf{1}}$ convergence of $\vec{W}^{h}$ to $\vec{W}$. To prove that $\vec{W}$ is a weak solution of the Cauchy problem (3.4a), (3.4b), it remains to show that

$$
\int_{\mathbb{R}_{+}} \int_{\mathbb{R}} p^{-1}(w-v)\left[\phi_{t}+v \phi_{x}\right]\left(\begin{array}{c}
1 \\
w
\end{array}\right) \mathrm{d} x \mathrm{~d} t=\overrightarrow{0}
$$

for any test function $\phi \in \mathbf{C}_{\mathbf{c}}^{\infty}\left(\mathbb{R}^{2} ; \mathbb{R}\right)$ with compact support contained in the half plane where $t>0$. Since $\vec{W}^{h}$ are uniformly bounded and $F$ is uniformly continuous on bounded sets, it suffices to prove that

$$
\lim _{h \rightarrow+\infty}\left[\int_{\mathbb{R}_{+}} \int_{\mathbb{R}} p^{-1}\left(w^{h}-v^{h}\right)\left[\phi_{t}+v^{h} \phi_{x}\right]\left(\begin{array}{c}
1 \\
w^{h}
\end{array}\right) \mathrm{d} x \mathrm{~d} t\right]=\overrightarrow{0} .
$$

Choose $T>0$ such that $\phi(t, x)=0$ whenever $t \notin] 0, T$. By the Green-Gauss formula the double integral above can be written as

$$
\int_{0}^{T} \sum_{i \in \mathcal{J}^{h}}\left[\dot{s}_{i}^{h}(t) \Delta \vec{Y}_{i}(t)-\Delta F_{i}(t)\right] \phi\left(t, s_{i}^{h}(t)\right) \mathrm{d} t
$$

where, with the same notation introduced in (5.6),

$$
\begin{aligned}
& \Delta \vec{Y}_{i}(t) \doteq p^{-1}\left(w_{i+\frac{1}{2}}^{h}-v_{i+\frac{1}{2}}^{h}\right)\left(\begin{array}{c}
1 \\
w_{i+\frac{1}{2}}^{h}
\end{array}\right)-p^{-1}\left(w_{i-\frac{1}{2}}^{h}-v_{i-\frac{1}{2}}^{h}\right)\left(\begin{array}{c}
1 \\
w_{i-\frac{1}{2}}^{h}
\end{array}\right), \\
& \Delta F_{i}(t) \doteq q\left(\vec{W}_{i+\frac{1}{2}}^{h}\right)\left(\begin{array}{c}
1 \\
w_{i+\frac{1}{2}}^{h}
\end{array}\right)-q\left(\vec{W}_{i-\frac{1}{2}}^{h}\right)\left(\begin{array}{c}
1 \\
w_{i-\frac{1}{2}}^{h}
\end{array}\right) .
\end{aligned}
$$

Observe that if $\vec{W}^{h}(t)$ performs a non-classical (stationary) shock, then $w_{-1 / 2}^{h}=w_{1 / 2}^{h}$ and by (5.2c)

$$
\lim _{h \rightarrow+\infty}\left\|\Delta F_{0}(t)\right\| \leq \lim _{h \rightarrow+\infty}\left|q\left(\check{W}^{h}\left(q_{0}(t), w_{\frac{1}{2}}^{h}\right)\right)-q\left(\hat{W}^{h}\left(q_{0}(t), w_{\frac{1}{2}}^{h}\right)\right)\right|\left(1+V_{0}\right)=0 .
$$

If the $i$ th discontinuity is not a non-classical shock, then by the Rankine-Hugoniot condition

$$
\left\|\dot{s}_{i}^{h}(t) \Delta \vec{Y}_{i}(t)-\Delta F_{i}(t)\right\|=0
$$

Therefore we have

$$
\lim _{h \rightarrow+\infty}\left\|\sum_{i \in \mathcal{J}^{h}}\left[\dot{s}_{i}^{h}(t) \Delta \vec{Y}_{i}(t)-\Delta F_{i}(t)\right] \phi\left(t, s_{i}^{h}(t)\right)\right\| \leq \lim _{h \rightarrow+\infty}\left\|\Delta F_{0}(t)\right\|\|\phi\|_{\infty}=0
$$

and this concludes the proof that $\vec{W}$ is a weak solution of (2.6).

It remains to justify that $\vec{W}$ is a constrained weak solution. By construction, we have that $q\left(\vec{W}^{h}\left(t, 0^{-}\right)\right) \leq q_{0}$ for any $t>0$. Yet it is not immediate to deduce that $\vec{W}$ satisfies (3.5), because the a.e. convergence of $\left(\vec{W}^{h}\right)_{h}$ to $\vec{W}$ does not ensure convergence of the corresponding traces on $x=0$. However, one can exploit the fact that both $\vec{W}^{h}$ and $\vec{W}$ are weak solutions of $(2.6)$ in the half-space $\mathbb{R}_{+} \times \mathbb{R}_{-}$. Then by applying the Green-Gauss formula to the weak formulation of the first equation of the system $(5.5 \mathrm{a}), \rho\left(\vec{W}^{h}\right)_{t}+q\left(\vec{W}^{h}\right)_{x}=0$, we find

$$
\int_{\mathbb{R}_{+}} q\left(\vec{W}^{h}\left(t, 0^{-}\right)\right) \psi(t) \mathrm{d} t=\int_{\mathbb{R}_{+}} \int_{\mathbb{R}_{-}}\left[\rho\left(\vec{W}^{h}\right) \dot{\psi}(t) \xi(x)+q\left(\vec{W}^{h}\right) \psi(t) \xi^{\prime}(x)\right] \mathrm{d} x \mathrm{~d} t
$$

where $\psi$ is an arbitrary $\mathbf{C}_{\mathbf{c}}^{\infty}$ test function of time with compact support in $] 0,+\infty\left[\right.$ and $\xi$ is some fixed $\mathbf{C}_{\mathbf{c}}^{\infty}$ test function of space such that $\xi(0)=1$. Now, the right-hand side passes to the limit, yielding the analogous expression with $\vec{W}^{h}$ replaced by $\vec{W}$. By using again the Green-Gauss formula, one finally finds that

$$
\lim _{h \rightarrow+\infty} \int_{\mathbb{R}_{+}} q\left(\vec{W}^{h}\left(t, 0^{-}\right)\right) \psi(t) \mathrm{d} t=\int_{\mathbb{R}_{+}} q\left(\vec{W}\left(t, 0^{-}\right)\right) \psi(t) \mathrm{d} t .
$$

Therefore the traces $\left(q\left(\vec{W}^{h}\left(t, 0^{-}\right)\right)\right)_{h}$ weakly converge to the trace $q\left(\vec{W}\left(t, 0^{-}\right)\right)$. Since weak convergence preserves pointwise inequalities, we have $q\left(\vec{W}\left(t, 0^{-}\right)\right) \leq q_{0}$. Finally, recall also that $q\left(\vec{W}\left(t, 0^{+}\right)\right)=q\left(\vec{W}\left(t, 0^{-}\right)\right)$because $\vec{W}$ is a weak solution in the whole $\mathbb{R}_{+} \times \mathbb{R}$. Therefore, the constraint relation (3.5) is inherited by $\vec{W}$ from the wave-front tracking approximations $\left(\vec{W}^{h}\right)_{h}$.

Proposition $9 \vec{W}$ is a constrained entropy solution of (3.4) in the sense of Definition 6. 
Proof In order to establish (3.6), it is enough to apply Proposition 7. Indeed, it is evident that due to the a.e. convergence of $\left(\vec{W}^{h}\right)_{h}$ to $\vec{W}$, the line (5.7a) yields precisely the first line (3.6a) of the desired entropy inequality. One has to find the second line $(3.6 \mathrm{~b})$ as the limit of the line $(5.7 \mathrm{~b})$; in other words, we have to justify that if $q_{0}>0$ (the case $q_{0}=0$ is trivial), as $h$ goes to infinity,

$$
\int_{\mathbb{R}_{+}} q\left(\vec{W}^{h}\left(t, 0^{+}\right)\right)\left[\frac{k}{q_{0}}-\frac{1}{p^{-1}\left(\left[w^{h}(t, 0)-k\right]^{+}\right)}\right]^{+} \phi(t, 0) \mathrm{d} t
$$

converges to

$$
\int_{\mathbb{R}_{+}} q(\vec{W}(t, 0))\left[\frac{k}{q_{0}}-\frac{1}{p^{-1}\left([w(t, 0)-k]^{+}\right)}\right]^{+} \phi(t, 0) \mathrm{d} t .
$$

Proposition 3 tells us that (5.13) coincides with (3.9), because $\vec{W}$ is a weak solution of ARZ. It is evident that (3.9) is the limit of the analogous term

$$
\int_{\mathbb{R}_{+}} \int_{\mathbb{R}_{-}}\left[\rho\left(\vec{W}^{h}\right) \mathfrak{f}_{k}\left(w^{h}\right) \psi_{t} \xi+q\left(\vec{W}^{h}\right) \mathfrak{f}_{k}\left(w^{h}\right) \psi \xi_{x}\right] \mathrm{d} x \mathrm{~d} t
$$

where, with the same notation introduced in Proposition 3, we denote $\mathfrak{f}_{k}(w) \doteq\left[\frac{k}{q_{0}}-\frac{1}{p^{-1}\left([w-k]^{+}\right)}\right]^{+}, \psi(t) \doteq \phi(t, 0)$ and $\xi$ is an arbitrary $\mathbf{C}_{\mathbf{c}}^{\infty}$ test function such that $\xi(0)=1$. However, it is not true in general that (5.14) equals (5.12), since $\vec{W}^{h}$ is a weak solution of (2.6) in $\mathbb{R}_{-}$and $\mathbb{R}_{+}$, but not in the whole $\mathbb{R}$. In fact, $\vec{W}^{h}$ may not satisfy the RankineHugoniot jump condition at $x=0$. Remarkably, thanks to the special structure of the ARZ system, we are able to prove that $W^{h}$ satisfies the property of renormalization as it is formulated in Remark 3 up to an error vanishing as $h$ goes to infinity. To do so, observe that we can sharpen (5.2c) to get for $\Delta q^{h}(t) \doteq q\left(\vec{W}^{h}\left(t, 0^{+}\right)\right)-q\left(\vec{W}^{h}\left(t, 0^{-}\right)\right)$ the estimate

$$
\left\|\Delta q^{h}\right\|_{\infty} \leq \epsilon(h)
$$

for some $\epsilon(h)$ that converges to zero as $h$ goes to infinity. By construction, $\vec{W}^{h}$ satisfies the following equations in the weak sense:

$$
\begin{aligned}
& \rho\left(\vec{W}^{h}\right)_{t}+q\left(\vec{W}^{h}\right)_{x}=\Delta q^{h}(t) \delta_{0}(x), \\
& {\left[\rho\left(\vec{W}^{h}\right) w^{h}\right]_{t}+\left[q\left(\vec{W}^{h}\right) w^{h}\right]_{x}=\Delta q^{h}(t) w^{h} \delta_{0}(x),}
\end{aligned}
$$

where $\delta_{0}$ is the Dirac delta distribution concentrated at zero. Observe that the right-hand side of the latter equation makes sense because $w^{h}$ have no jump across $x=0$, unless $q\left(\vec{W}^{h}\left(t, 0^{+}\right)\right)=0=q\left(\vec{W}^{h}\left(t, 0^{-}\right)\right)$. Thus $\Delta q^{h}(t) w^{h}(t, 0)$ is zero whenever the definition of $w^{h}(t, 0)$ is ambiguous. Now, we define a divergence-free field $\left(A^{h}, B^{h}\right)$ by

$$
A^{h}(t, x) \doteq \rho\left(\vec{W}^{h}(t, x)\right)+\epsilon(h), \quad \quad B^{h}(t, x) \doteq q\left(\vec{W}^{h}(t, x)\right)-\Delta q^{h}(t) \Theta(x),
$$

where $\Theta$ stands for the Heavyside function (we have $\Theta^{\prime}=\delta_{0}$ ). Due to the estimates (5.15) and $\left|q\left(\vec{W}^{h}\right)\right| \leq V_{0} \rho\left(\vec{W}^{h}\right)$, we see that the condition $\left|B^{h}\right| \leq$ const $A^{h}$ needed to apply the theory in [37] is fulfilled. Now, the equation $\left(A^{h} w^{h}\right)_{t}+\left(B^{h} w^{h}\right)_{x}=0$ is satisfied up to a measure source term, more precisely we have

$$
\left(A^{h} w^{h}\right)_{t}+\left(B^{h} w^{h}\right)_{x}=\mu^{h}(t, x),
$$

where

$$
\mu^{h}(t, x) \doteq \epsilon(h) w_{t}^{h}-\Theta(x) \Delta q^{h}(t) w_{x}^{h}
$$

Observe that $\left(\mu^{h}\right)_{h}$ is a sequence of measures that vanishes as $h$ goes to infinity. Indeed, beside (5.15), by (3.11) we have uniform in $h$ estimates of the total variation of $\left(w^{h}\right)_{h}$ both in space and time.

The theory in [37] does not apply directly to equations with measure valued source terms. Fortunately, in our case the coefficients $A^{h}, B^{h}$ of the continuity equation and the solution $w^{h}$ are piecewise constant functions with discontinuities laying along a finite number of straight line segments in the $(t, x)$-plane. Therefore the renormalization property can be verified by a direct calculation. As a result, from (5.16) (understood in the sense of distributions) we get the renormalized inequalities

$$
\left|\left[A^{h} \mathfrak{f}_{k}\left(w^{h}\right)\right]_{t}+\left[B^{h} \mathfrak{f}_{k}\left(w^{h}\right)\right]_{x}\right| \leq\left\|\mathfrak{f}_{k}^{\prime}\right\|_{\infty} \epsilon(h)\left[\left|w_{t}^{h}\right|+\left|w_{x}^{h}\right|\right] \rightarrow 0
$$

(in the sense of distributions). Let us give some detail of the calculation. If a portion of straight line segment $\Gamma$ of slope $\gamma$ in the $(t, x)$-plane separates the states $w_{-}^{h}$ (at the left) and $w_{+}^{h}$ (at the right), and the respective coefficients of the continuity equation take values $A_{ \pm}^{h}, B_{ \pm}^{h}$, respectively, then we have

$$
-\gamma A_{+}^{h}+B_{+}^{h}=-\gamma A_{-}^{h}+B_{-}^{h}
$$


because the field $\left(A^{h}, B^{h}\right)$ is divergence-free by construction. Then $(5.16)$ yields $\left(-\gamma A_{ \pm}^{h}+B_{ \pm}^{h}\right)\left(w_{+}-w_{-}\right)=r^{h}(\Gamma)$ where $r^{h}(\Gamma)$ corresponds to the contribution of the jump across $\Gamma$ to the measure $\mu^{h}$. Hence we have

$$
\left|\left(-\gamma A_{ \pm}^{h}+B_{ \pm}^{h}\right)\left(\mathfrak{f}_{k}\left(w_{+}\right)-\mathfrak{f}_{k}\left(w_{-}\right)\right)\right| \leq\left\|\mathfrak{f}_{k}^{\prime}\right\|_{\infty}\left|r^{h}(\Gamma)\right|
$$

due to the elementary estimate

$$
\left|\mathfrak{f}_{k}\left(w_{+}^{h}\right)-\mathfrak{f}_{k}\left(w_{-}^{h}\right)\right| \leq\left\|\mathfrak{f}_{k}^{\prime}\right\|_{\infty}\left|w_{+}^{h}-w_{-}^{h}\right| .
$$

Notice that this calculation applies as well to the case where $w^{h}$ is continuous across $\Gamma$ but only $A^{h}$ and $B^{h}$ differ. Now, observe that by (5.18) and (5.19)

$$
\left|-\gamma\left(A_{+}^{h} \mathfrak{f}_{k}\left(w_{+}^{h}\right)-A_{-}^{h} \mathfrak{f}_{k}\left(w_{-}^{h}\right)\right)+\left(B_{+}^{h} \mathfrak{f}_{k}\left(w_{+}^{h}\right)-B_{-}^{h} \mathfrak{f}_{k}\left(w_{-}^{h}\right)\right)\right| \leq\left\|\mathfrak{f}_{k}^{\prime}\right\|_{\infty}\left|r^{h}(\Gamma)\right| .
$$

After summation over all jumps $\Gamma$, the terms $r^{h}(\Gamma)$ make appear the total variation of $\mu^{h}$ on the right-hand side. Being understood that the coefficients $A^{h}, B^{h}$ and solutions $w^{h}$ are piecewise constant, we find precisely (5.17).

Now, applying the Green-Gauss formula to (5.17), one finds that (5.14) and (5.12) differ by a term that vanishes as $h$ goes to infinity. Eventually, this justifies the fact that (5.12) converges to (5.13).

\section{The Cauchy problem with time-dependent constraint: Proof of Theorem 1}

For the case of piecewise constant constraint $q_{0}$ that varies in time, we only have to adapt the interaction estimates to take into account changes in the profile that occur when the constraint level changes. Indeed, instantaneous changes of the constraint level may lead to the appearance or the demise of a non-classical shock in the solution constructed by the wave-front tracking procedure of the previous section.

We call $t_{i}>0, i \in\{1, \ldots, N\}$, the times at which $q_{0}$ changes value. Then let $t_{0} \doteq 0, t_{N+1} \doteq+\infty$ and $q_{0}(t) \doteq q_{i+1}$ for all $t \in\left[t_{i}, t_{i+1}\left[\right.\right.$, with $q_{i+1} \in\left[0, \dot{q}\left(V_{0}\right)\right]$ and $q_{i+1} \neq q_{i+2}$.

Note that in principle, it is possible to prove the statement of Theorem 1 without a detailed study of the "interactions" at $x=0$ due to the jumps at times $t=t_{i}$ in the level of the constraint. Indeed, the assumption $\max _{i \in\{1, \ldots, N+1\}} \operatorname{TV}\left(J\left(q_{i}, w_{0}\right) ; \mathbb{R}_{-}\right)<+\infty$ would be sufficient in order to restart the construction of approximate solutions at every time $t=t_{i}$ and to obtain a finite bound, uniform in $h$ and $t$, on the variation of $x \mapsto \vec{W}^{h}(t, x)$. In the present paper we make the choice to carefully keep track of the possible increase in the total variation, as the details we provide below explain why it is not at all clear how to extend the result of Theorem 1 to a general time-dependent constraint function $t \mapsto q_{0}(t)$.

Fix $h \in \mathbb{N}$ sufficiently large and take $\varepsilon^{h} \doteq 2^{-h} V_{0}$, where $V_{0} \doteq\left\|\vec{W}_{0}\right\|_{\infty}$. In the sequel we will use the same notations introduced in Section 5.1. In particular we approximate the constrained states $\mathcal{C}\left(q_{i}\right)$ by $\mathcal{C}^{h}\left(q_{i}\right)$ and at every time $t \in] t_{i}, t_{i+1}\left[, i \in\{1, \ldots, N\}\right.$, we use properties (5.2) with $q_{i+1}$ replacing $q_{0}$. Moreover, for $h$ sufficiently large, we can assume that

$$
J^{h}\left(q_{i}, V_{0}\right) \neq J^{h}\left(q_{i+1}, V_{0}\right) .
$$

In the sequel we will need the following hypothesis on the initial condition $\vec{W}_{0}$ and the constraint function $q_{0}$ :

$$
\left.\left.\left.\left.K_{0} \doteq \sum_{i=1}^{N}\left[\sup _{y \in \mathbb{R}_{-}} \mid \operatorname{TV}\left(J\left(q_{i}, w_{0}\right) ;\right]-\infty, y\right]\right)-\operatorname{TV}\left(J\left(q_{i+1}, w_{0}\right) ;\right]-\infty, y\right]\right) \mid\right]<+\infty
$$

Due to the fact that the set of values $i$ corresponding to the times $t=t_{i}$ is finite, we can use the continuity of $J\left(q_{i}, \cdot\right)$, the convergence of $J^{h}\left(q_{i}, \cdot\right)$ to $J\left(q_{i}, \cdot\right)$ as $h$ goes to infinity, and the semi-continuity of the total variation functional TV with respect to the a.e. on $\mathbb{R}$ convergence of sequences of functions, to approximate $\vec{W}_{0}$ with $\vec{W}_{0}^{h} \in \mathbf{P C}\left(\mathbb{R} ; \mathcal{W}^{h}\right)$ such that (5.3) and (2.15) are satisfied together with

$$
\begin{aligned}
& \sum_{i=1}^{N}\left|J^{h}\left(q_{i}, V_{0}\right)-J^{h}\left(q_{i+1}, V_{0}\right)\right| \leq K_{1}, \\
& \left.\left.\left.\left.\sum_{i=1}^{N}\left[\sup _{y \in \mathbb{R}_{-}} \mid \operatorname{TV}\left(J^{h}\left(q_{i}, w_{0}^{h}\right) ;\right]-\infty, y\right]\right)-\operatorname{TV}\left(J^{h}\left(q_{i+1}, w_{0}^{h}\right) ;\right]-\infty, y\right]\right) \mid\right] \leq K_{2},
\end{aligned}
$$

where the values of $K_{1}$ and $K_{2}$ may depend on $K_{0}$, but are uniform with respect to $h$. The constants $K_{1}$ and $K_{2}$ allow us to quantify the increase of $\operatorname{TV}\left(\vec{W}^{h}(t, \cdot)\right)$ due to jumps in the constraint level. Then we obtain the approximate constrained Cauchy problem

$$
\begin{array}{ll}
Y(\vec{W})_{t}+F(Y(\vec{W}))_{x}=0, & t>0, x \in \mathbb{R}, \\
\vec{W}(0, x)=\vec{W}_{0}^{h}(x), & x \in \mathbb{R},
\end{array}
$$




$$
\vec{W}\left(t, 0^{ \pm}\right) \notin \mathcal{C}^{h}\left(q_{i+1}\right), \quad t \in\left[t_{i}, t_{i+1}[.\right.
$$

Now we can start with the construction of an approximate solution $t \mapsto \vec{W}^{h}(t)$ to the above problem. For $\left.t \in] 0, t_{1}\right]$, the approximate solution $t \mapsto \vec{W}^{h}(t)$ is obtained by applying the same construction as in Section 5.1 and using $\mathcal{R S}^{h}$ and $\mathcal{R S}_{q_{1}}^{h}$. Then the approximate solution $t \mapsto \vec{W}^{h}(t)$ is constructed for $\left.\left.t \in\right] t_{1}, t_{2}\right]$ by applying the same procedure with $\vec{W}^{h}\left(t_{1}\right)$ instead of $\vec{W}_{0}^{h}$ as initial datum and $\mathcal{R} \mathcal{S}_{q_{2}}^{h}$ instead of $\mathcal{R} \mathcal{S}_{q_{1}}^{h}$ as approximate constrained Riemann solver.

Roughly speaking, as long as $q_{0}$ takes the value $q_{*}$, we apply the procedure explained in Section 5.1 by using $\mathcal{R S}_{q_{*}}^{h}$ in $x=0$ and $\mathcal{R S}^{h}$ away from $x=0$. More rigorously, the present algorithm consists in the application of two operators, $\mathcal{T}$ and $\mathcal{S}$, the first operator gives $\mathcal{T}\left[\vec{W}^{h}\right]\left(t_{i}\right)=\vec{W}^{h}\left(t_{i}\right) \in \mathbf{P C}\left(\mathbb{R} ; \mathcal{W}^{h}\right)$, while the second operator gives the approximate solution $\mathcal{S}\left[\vec{W}_{*}^{h}, q_{*}\right]$ of the problem

$$
\begin{array}{ll}
Y(\vec{W})_{t}+F(Y(\vec{W}))_{x}=0, & t>0, x \in \mathbb{R}, \\
\vec{W}(0, x)=\vec{W}_{*}^{h}(x), & x \in \mathbb{R}, \\
q\left(\vec{W}\left(t, 0^{ \pm}\right)\right) \leq q_{*}, & t>0 .
\end{array}
$$

Then we define recursively

$$
\begin{array}{lll}
\mathcal{F}\left[\vec{W}_{0}^{h}\right](t) \doteq \mathcal{S}\left[\vec{W}_{0}^{h}, q_{1}\right](t) & \text { if } \left.t \in] 0, t_{1}\right], & \\
\mathcal{F}\left[\vec{W}_{0}^{h}\right](t) \doteq \mathcal{S}\left[\mathcal{T}\left[\mathcal{F}\left[\vec{W}_{0}^{h}\right]\right]\left(t_{i}\right), q_{i+1}\right]\left(t-t_{i}\right) & \text { if } \left.t \in] t_{i}, t_{i+1}\right], & i \in\{1, \ldots, N-1\}, \\
\mathcal{F}\left[\vec{W}_{0}^{h}\right](t) \doteq \mathcal{S}\left[\mathcal{T}\left[\mathcal{F}\left[\vec{W}_{0}^{h}\right]\right]\left(t_{N}\right), q_{N+1}\right]\left(t-t_{N}\right) & \text { if } t \in] t_{N},+\infty[. &
\end{array}
$$

In Section 6.1 we prove that $\vec{W}^{h}(t)$ is well defined for all $t>0$ and belongs to $\mathbf{P C}\left(\mathbb{R} ; \mathcal{W}^{h}\right)$. Finally, in Section 6.2 we prove that $\left(\vec{W}^{h}\right)_{h}$ converges (along a subsequence) in $\mathbf{L}_{\text {loc }}^{1}$ to $\vec{W}$ and prove that $\vec{W}$ is in fact a constrained entropy solution to (3.4) in the sense of Definition 6.

\subsection{A priori estimates}

In this section we prove that $\vec{W}^{h}(t)=\left(v^{h}(t), w^{h}(t)\right)^{T}$ takes values in $\mathcal{W}^{h}$ and we estimate $\operatorname{TV}\left(\vec{W}^{h}(t)\right)$ uniformly in $h$ and $t$. To this aim we consider the Temple type functional

$$
\begin{aligned}
\mathcal{L}(t)= & \mathcal{B}(t)+3 \sum_{\substack{i \in\{1, \ldots, N\} \\
\text { s.t. } t_{i} \geq t}}\left[\left|J^{h}\left(q_{i}, V_{0}\right)-J^{h}\left(q_{i+1}, V_{0}\right)\right|\right] \\
& \left.\left.\left.\left.+3 \sum_{\substack{i \in\{1, \ldots, N\} \\
\text { s.t. } t_{i} \geq t}}\left[\sup _{y \in \mathbb{R}_{-}} \mid \operatorname{TV}\left(J^{h}\left(q_{i}, w_{0}^{h}\right) ;\right]-\infty, y\right]\right)-\operatorname{TV}\left(J^{h}\left(q_{i+1}, w_{0}^{h}\right) ;\right]-\infty, y\right]\right) \mid\right] .
\end{aligned}
$$

In the expression of $\mathcal{L}$, the terms added to $\mathcal{B}$ account for the time dependence of $q_{0}$.

Proposition 10 For any fixed $h \in \mathbb{N}, \vec{W}_{0}^{h} \in \mathbf{P C}\left(\mathbb{R} ; \mathcal{W}^{h}\right)$, we have that:

1. $\vec{W}^{h}(t) \in \mathbf{P C}\left(\mathbb{R} ; \mathcal{W}^{h}\right)$ for all $t>0$;

2. the map $t \mapsto \mathcal{L}(t)$ is non increasing and it decreases by at least $\varepsilon^{h}$ each time the number of waves increases.

Proof The proof is based on the extension of the results already achieved in the proof of Proposition 6 to the case of a time depending constraint. We will use the same notations introduced in the proof of Proposition 6 and prove that the map $t \mapsto \mathcal{L}(t)$ is non-increasing and it decreases by at least $\varepsilon^{h}$ each time the number of waves increases. For any $\bar{t} \in] t_{i}, t_{i+1}[$ we know that $\Delta \mathcal{L} \doteq \mathcal{L}(\bar{t}+)-\mathcal{L}(\bar{t}-)=\Delta \mathcal{B}$ and therefore we can apply the results obtained in the proof of Proposition 6 .

If $\bar{t}=t_{i}>0$, then the constraint $q_{0}$ changes value from $q_{i}$ to $q_{i+1}$. Recall that by assumption both $q_{i}$ and $q_{i+1}$ are strictly positive. For simplicity, assume that at time $t=\bar{t}$ no interaction takes place. Then $w^{h}(\bar{t}-) \equiv w^{h}(\bar{t}+) \equiv$ $\bar{w} \in \mathbf{P C}\left(\mathbb{R} ; \varepsilon^{h} \mathbb{N}\right)$. An important ingredient of the proof is the observation that for all $t>0, \bar{w}$ on $\left.]-\infty, 0\right]$ is a piecewise constant function which takes the same values - and in the same order - as the initial function $w_{0}^{h}$ restricted to some interval ] $\left.-\infty, y^{h}(t)\right]$ with $y^{h}(t) \leq 0$. This readily follows from the linear degeneracy of the second characteristic field and from the fact that the speeds of all waves of the second family are non-negative. Therefore, the values in the range of $w_{0}^{h}$ on $\mathbb{R}$ do not disappear from the solution because of the interactions, but they can leave the domain of interest $\mathbb{R}_{-}$in finite time. The curve $y^{h}$ is implicitly defined from the fact that $\operatorname{TV}\left(J^{h}(q, \bar{w}) ; \mathbb{R}_{-}\right)$equals $\left.\left.\operatorname{TV}\left(J^{h}\left(q, w_{0}\right) ;\right]-\infty, y^{h}(t)\right]\right)$ for all $q \geq 0$.

Now we are ready to examine the increase of $\mathcal{L}$ at times $t_{i}$. 
(C.1) If the solution performs a non-classical shock before time $t=\bar{t}$, namely $\vec{W}_{-1 / 2}^{h}=\hat{W}_{1 / 2}$ and $\vec{W}_{1 / 2}^{h}=\check{W}_{1 / 2}$, then we have to distinguish three cases.

(C.1a) If $\mathcal{R S}_{q_{i+1}}\left[\hat{W}_{1 / 2}, \breve{W}_{1 / 2}\right]$ performs a discretized rarefaction, then $\Delta \gamma=J^{h}\left(q_{i+1}, w_{1 / 2}\right)=0$, the number of waves increases and by (6.1)

$$
\begin{aligned}
\Delta \mathcal{L}= & 3\left[\operatorname{TV}\left(J^{h}\left(q_{i+1}, \bar{w}\right) ; \mathbb{R}_{-}\right)-\operatorname{TV}\left(J^{h}\left(q_{i}, \bar{w}\right) ; \mathbb{R}_{-}\right)\right]-3\left|J^{h}\left(q_{i+1}, V_{0}\right)-J^{h}\left(q_{i}, V_{0}\right)\right| \\
& \left.\left.\left.\left.-3 \sup _{y \in \mathbb{R}_{-}} \mid \operatorname{TV}\left(J^{h}\left(q_{i+1}, w_{0}^{h}\right) ;\right]-\infty, y\right]\right)-\operatorname{TV}\left(J^{h}\left(q_{i}, w_{0}^{h}\right) ;\right]-\infty, y\right]\right) \mid \\
\leq & -3\left|J^{h}\left(q_{i+1}, V_{0}\right)-J^{h}\left(q_{i}, V_{0}\right)\right|<-\varepsilon .
\end{aligned}
$$

(C.1b) If $\mathcal{R S}_{q_{i+1}}\left[\hat{W}_{1 / 2}, \check{W}_{1 / 2}\right]$ performs a possible null discretized rarefaction in $\mathbb{R}_{-}$, a non-classical shock and a discretized rarefaction in $\mathbb{R}_{+}$, then $\Delta \gamma=0$, the number of waves increases and by (6.1)

$$
\begin{aligned}
\Delta \mathcal{L}= & 3\left[\operatorname{TV}\left(J^{h}\left(q_{i+1}, \bar{w}\right) ; \mathbb{R}_{-}\right)-\operatorname{TV}\left(J^{h}\left(q_{i}, \bar{w}\right) ; \mathbb{R}_{-}\right)\right]-3\left|J^{h}\left(q_{i+1}, V_{0}\right)-J^{h}\left(q_{i}, V_{0}\right)\right| \\
& \left.\left.\left.\left.-3 \sup _{y \in \mathbb{R}_{-}} \mid \operatorname{TV}\left(J^{h}\left(q_{i+1}, w_{0}^{h}\right) ;\right]-\infty, y\right]\right)-\operatorname{TV}\left(J^{h}\left(q_{i}, w_{0}^{h}\right) ;\right]-\infty, y\right]\right) \mid \\
\leq & -3\left|J^{h}\left(q_{i+1}, V_{0}\right)-J^{h}\left(q_{i}, V_{0}\right)\right|<-\varepsilon .
\end{aligned}
$$

(C.1c) If $\mathcal{R S}_{q_{i+1}}\left[\hat{W}_{1 / 2}, \check{W}_{1 / 2}\right]$ performs a possible null shock in $\mathbb{R}_{-}$, a non-classical shock and a shock in $\mathbb{R}_{+}$, then $\Delta \gamma=0$, the number of waves increases and by $(6.1)$

$$
\begin{aligned}
\Delta \mathcal{L}= & 2\left[J^{h}\left(q_{i+1}, w_{\frac{1}{2}}\right)-J^{h}\left(q_{i}, w_{\frac{1}{2}}\right)\right]+3\left[\operatorname{TV}\left(J^{h}\left(q_{i+1},, \bar{w}\right) ; \mathbb{R}_{-}\right)-\operatorname{TV}\left(J^{h}\left(q_{i}, \bar{w}\right) ; \mathbb{R}_{-}\right)\right] \\
& \left.\left.\left.\left.-3\left|J^{h}\left(q_{i+1}, V_{0}\right)-J^{h}\left(q_{i}, V_{0}\right)\right|-3 \sup _{y \in \mathbb{R}_{-}} \mid \operatorname{TV}\left(J^{h}\left(q_{i+1}, w_{0}^{h}\right) ;\right]-\infty, y\right]\right)-\operatorname{TV}\left(J^{h}\left(q_{i}, w_{0}^{h}\right) ;\right]-\infty, y\right]\right) \mid \\
\leq & -\left[J^{h}\left(q_{i+1}, V_{0}\right)-J^{h}\left(q_{i}, V_{0}\right)\right] \leq-\varepsilon .
\end{aligned}
$$

(C.2) If the solution performs a stationary wave of the first family along $x=0$ before time $t=\bar{t}$, namely $w_{-1 / 2}=w_{1 / 2}, v_{-1 / 2} \neq v_{1 / 2}$ and $q\left(\vec{W}_{-1 / 2}^{h}\right)=q\left(\vec{W}_{1 / 2}^{h}\right)$, and $\mathcal{R S}_{q_{i+1}}\left[\vec{W}_{-1 / 2}, \vec{W}_{1 / 2}\right]$ performs a possible null shock in $\mathbb{R}_{-}$, a non-classical shock and a shock in $\mathbb{R}_{+}$, namely $v_{ \pm 1 / 2} \in\left[\hat{v}^{h}\left(q_{i+1}, w_{1 / 2}\right), \check{v}^{h}\left(q_{i+1}, w_{1 / 2}\right)[\right.$, then $\Delta \gamma=-2 J^{h}\left(q_{i}, w_{1 / 2}\right)$, the number of waves increases and by $(6.1)$

$$
\begin{aligned}
\Delta \mathcal{L} \leq & 2 J^{h}\left(q_{i+1}, w_{\frac{1}{2}}\right)+3\left[\operatorname{TV}\left(J^{h}\left(q_{i+1}, \bar{w}\right) ; \mathbb{R}_{-}\right)-\operatorname{TV}\left(J^{h}\left(q_{i}, \bar{w}\right) ; \mathbb{R}_{-}\right)\right]-2 J^{h}\left(q_{i}, w_{\frac{1}{2}}\right) \\
& \left.\left.\left.\left.-3\left|J^{h}\left(q_{i+1}, V_{0}\right)-J^{h}\left(q_{i}, V_{0}\right)\right|-3 \sup _{y \in \mathbb{R}_{-}} \mid \operatorname{TV}\left(J^{h}\left(q_{i+1}, w_{0}^{h}\right) ;\right]-\infty, y\right]\right)-\operatorname{TV}\left(J^{h}\left(q_{i}, w_{0}^{h}\right) ;\right]-\infty, y\right]\right) \mid \\
\leq & -\left[J^{h}\left(q_{i+1}, V_{0}\right)-J^{h}\left(q_{i}, V_{0}\right)\right] \leq-\varepsilon .
\end{aligned}
$$

(C.3) If the solution is continuous along $x=0$ before time $t=\bar{t}$, namely $\vec{W}_{-1 / 2}=\vec{W}_{1 / 2}$, and $\mathcal{R} \mathcal{S}_{q_{i+1}}\left[\vec{W}_{-1 / 2}, \vec{W}_{1 / 2}\right]$ performs a non-classical shock and one shock on each side of $x=0$, then $\Delta \gamma=-2 J^{h}\left(q_{i}, w_{1 / 2}\right)$, the number of waves increases and by (6.1)

$$
\begin{aligned}
\Delta \mathcal{L}= & 2 J^{h}\left(q_{i+1}, w_{\frac{1}{2}}\right)+3\left[\operatorname{TV}\left(J^{h}\left(q_{i+1}, \bar{w}\right) ; \mathbb{R}_{-}\right)-\operatorname{TV}\left(J^{h}\left(q_{i}, \bar{w}\right) ; \mathbb{R}_{-}\right)\right]-2 J^{h}\left(q_{i}, w_{\frac{1}{2}}\right) \\
& \left.\left.\left.\left.-3\left|J^{h}\left(q_{i+1}, V_{0}\right)-J^{h}\left(q_{i}, V_{0}\right)\right|-3 \sup _{y \in \mathbb{R}_{-}} \mid \operatorname{TV}\left(J^{h}\left(q_{i+1}, w_{0}^{h}\right) ;\right]-\infty, y\right]\right)-\operatorname{TV}\left(J^{h}\left(q_{i}, w_{0}^{h}\right) ;\right]-\infty, y\right]\right) \mid \\
\leq & -\left[J^{h}\left(q_{i+1}, V_{0}\right)-J^{h}\left(q_{i}, V_{0}\right)\right] \leq-\varepsilon .
\end{aligned}
$$

In the remaining cases the jump of $q_{0}$ does not change the solution and it is straightforward to prove that $\Delta \mathcal{L} \leq 0$. This concludes the proof.

\subsection{Convergence}

In this section we prove that $\left(\vec{W}^{h}\right)_{h}$ converges (along a subsequence) in $\mathbf{L}_{\text {loc }}^{1}$ to $\vec{W}$ and prove that $\vec{W}$ is in fact a constrained entropy solution of (2.5) in the sense of Definition 5.

By Proposition 10, (5.3), (6.2) and (6.3) we have that for any $t>0$

$$
\mathrm{TV}\left(\vec{W}^{h}(t)\right) \leq \mathcal{L}(t) \leq \mathcal{L}(0) \leq C,
$$


where

$$
C \doteq \mathrm{TV}\left(\vec{W}_{0}\right)+3 \mathrm{TV}\left(J\left(q_{0}(0), w_{0}\right) ; \mathbb{R}_{-}\right)+2 V_{0}+3 K_{1}+3 K_{2},
$$

with $J$ defined in (3.3). Moreover, observe that $\left\|\vec{W}^{h}(t)\right\|_{\infty} \leq V_{0}$ and

$$
\left\|\vec{W}^{h}(t)-\vec{W}^{h}(s)\right\|_{1} \leq L|t-s|
$$

with $L \doteq C \max \left\{V_{0}, p^{-1}\left(V_{0}\right) p^{\prime}\left(p^{-1}\left(V_{0}\right)\right)\right\}$. Thus, by applying Helly's Theorem in the form [9, Theorem 2.4], there exists a function $\vec{W} \in \mathbf{L}_{\text {loc }}^{1}\left(\mathbb{R}_{+} \times \mathbb{R} ;\left[0, V_{0}\right]^{2}\right)$ and a subsequence, still denoted $\left(\vec{W}^{h}\right)_{h}$, such that $\left(\vec{W}^{h}\right)_{h}$ converges to $\vec{W}$ in $\mathbf{L}_{\text {loc }}^{\mathbf{1}}\left(\mathbb{R}_{+} \times \mathbb{R} ;\left[0, V_{0}\right]^{2}\right)$ as $h$ goes to infinity. Moreover, $\vec{W}$ satisfies the estimates (3.11), namely for all $t, s \in \mathbb{R}_{+}$

$$
\operatorname{TV}(\vec{W}(t)) \leq C, \quad\|\vec{W}(t)-\vec{W}(s)\|_{1} \leq L|t-s|, \quad\|\vec{W}(t)\|_{\infty} \leq V_{0} .
$$

Remark 7 Given the result we obtain in this paper, the most natural approach to the case of a general timedependent constraint $q_{0}$ would be to approximate $q_{0}$ by a piecewise constant function $q_{0}^{\Delta t}$, constant on intervals of the form $[i \Delta t,(i+1) \Delta t[$. For simplicity one could set $h=\Delta t$ and then try to find an approximate initial condition $\vec{W}_{0}^{h}$ satisfying conditions analogous to (6.2) and (6.3). However, it is not clear to the authors which assumptions on $\vec{W}_{0}$ and $q_{0}$ may ensure the existence of approximating sequences $q_{0}^{h}$ and $\vec{W}_{0}^{h}$ verifying the condition (6.3) uniformly in $h$. Observe that asking this condition is similar to ask for a uniform bound of the quantity

$$
\operatorname{TV}_{t, x}\left(J^{h}\left(q_{0}, w_{0}^{h}\right) ; \mathbb{R}_{+} \times \mathbb{R}_{-}\right) .
$$

The singularity of $J\left(q_{0}, \cdot\right)$ at $w=\tilde{w}\left(q_{0}\right)$, pointed out in Lemma 2, makes it particularly delicate to discretize $t \mapsto q_{0}(t)$ and $x \mapsto w_{0}(x)$ in a compatible way. For this reason the study of the Cauchy problem for the ARZ system subject to a general time-dependent constraint is postponed to a future work, in which a different approach will be used.

Proposition 11 The function $\vec{W}$ is a constrained weak solution of (3.4) in the sense of Definition 5.

Proof The proof is the same as for the constant $q_{0}$ case, being understood that we have for discrete solutions $q\left(\vec{W}^{h}\left(0^{-}, t\right)\right) \leq q_{0}(t)$, that (in the same way as shown in Proposition 8 ) the left-hand side of this inequality converges weakly-* in $\mathbf{L}^{\infty}(] 0,+\infty\left[; \mathbb{R}_{+}\right)$to $q\left(\vec{W}\left(0^{-}, t\right)\right)$ as $h$ goes to infinity.

Proposition 12 The function $\vec{W}$ is a constrained entropy solution of (3.4) in the sense of Definition 6.

Proof The proof is essentially the same as in Proposition 9. The only potentially delicate part is the passage to the limit in the terms corresponding to the integral term in (3.6b) of the entropy formulation. Still, the fact that $q_{0}$ is a piecewise constant function makes this generalization rather straightforward. Indeed, we have $q_{0}=$ $\sum_{i=0}^{N} q_{i+1} \chi_{\left[t_{i}, t_{i+1} \text { [ }\right.}$ for some constant values $q_{i+1}$ and we can modify the notation of Proposition 3 by saying that for all $t \in\left[t_{i}, t_{i+1}[\right.$ we have

$$
\mathfrak{f}_{k}\left(q_{0}(t), w\right) \doteq \mathfrak{f}_{k}^{i}(t, w) \doteq\left[\frac{k}{q_{i+1}}-\frac{1}{p^{-1}\left([w-k]^{+}\right)}\right]^{+}
$$

For each $i \in \mathbb{N}$ we can pass to the limit, as $h$ goes to infinity, in the integral

$$
\int_{t_{i}}^{t_{i+1}}\left[q\left(\vec{W}^{h}\right) \mathfrak{f}_{k}^{i}\left(w^{h}\right)-q(\vec{W}) \mathfrak{f}_{k}^{i}(w)\right] \phi(t, 0) \mathrm{d} t
$$

exactly like in the proof of Proposition 9. Then, we can write $\int_{\mathbb{R}_{+}}$as $\sum_{i=0}^{N} \int_{t_{i}}^{t_{i+1}}$, the sum being finite because we integrate a compactly supported function. We conclude that

$$
\lim _{h \rightarrow+\infty} \int_{\mathbb{R}_{+}}\left[q\left(\vec{W}^{h}\right) \mathfrak{f}_{k}\left(q_{0}(t), w^{h}\right)-q(\vec{W}) \mathfrak{f}_{k}\left(q_{0}(t), w\right)\right] \phi(t, 0) \mathrm{d} t=0 .
$$

In conclusion, at the limit as $h$ goes to infinity we have found the required entropy inequalities (3.6).

Acknowledgements The first author warmly thanks LJLL, University Paris 6, and IRMAR, University of Rennes, for the hospitality during the preparation of this paper. The last author warmly thanks GSSI for the hospitality during the preparation of this paper. 


\section{References}

1. Amadori, D., Shen, W.: An Integro-Differential Conservation Law arising in a Model of Granular Flow. J. Hyperbolic Differ. Equ. 09(01), 105-131 (2012)

2. Andreianov, B., Donadello, C., Razafison, U., Rosini, M.D.: Riemann problems with non-local point constraints and capacity drop. Mathematical Biosciences and Engineering 12(2), 259-278 (2015)

3. Andreianov, B., Donadello, C., Rosini, M.D.: Crowd dynamics and conservation laws with nonlocal constraints and capacity drop. Mathematical Models and Methods in Applied Sciences 24(13), 2685-2722 (2014)

4. Andreianov, B., Goatin, P., Seguin, N.: Finite volume schemes for locally constrained conservation laws. Numerische Mathematik 115, 609-645 (2010)

5. Aw, A., Rascle, M.: Resurrection of "Second Order" Models of Traffic Flow. SIAM Journal on Applied Mathematics 60(3), pp. $916-938$ (2000)

6. Bardos, C., Leroux, A.Y., Nedelec, J.C.: First order quasilinear equations with boundary conditions. Communications in partial differential equations 4(9), 1017-1034 (1979)

7. Bellomo, N., Dogbe, C.: On the modeling of traffic and crowds: A survey of models, speculations, and perspectives. SIAM review 53(3), 409-463 (2011)

8. Bianchini, S., Bressan, A.: Vanishing viscosity solutions of nonlinear hyperbolic systems. Annals of Mathematics 161(1), 223-342 (2005)

9. Bressan, A.: Hyperbolic systems of conservation laws, Oxford Lecture Series in Mathematics and its Applications, vol. 20. Oxford University Press, Oxford (2000)

10. Bressan, A., Goatin, P.: Oleinik type estimates and uniqueness for $n \times n$ conservation laws. Journal of Differential Equations 156(1), $26-49(1999)$

11. Bressan, A., LeFloch, P.: Uniqueness of weak solutions to systems of conservation laws. Archive for Rational Mechanics and Analysis 140(4), 301-317 (1997)

12. Cancès, C., Seguin, N.: Error Estimate for Godunov Approximation of Locally Constrained Conservation Laws. SIAM Journal on Numerical Analysis 50(6), 3036-3060 (2012)

13. Chalons, C., Goatin, P., Seguin, N.: General constrained conservation laws. Application to pedestrian flow modeling. Networks and Heterogeneous Media 8(2), 433-463 (2013)

14. Chen, G.Q., Frid, H.: Divergence-measure fields and hyperbolic conservation laws. Arch. Ration. Mech. Anal. 147(2), 89-118 (1999)

15. Colombo, R.M., Goatin, P.: A well posed conservation law with a variable unilateral constraint. J. Differential Equations 234(2), 654-675 (2007)

16. Colombo, R.M., Goatin, P., Rosini, M.D.: Conservation laws with unilateral constraints in traffic modeling. In: Transport Management and Land-Use Effects in Presence of Unusual Demand, Atti del convegno SIDT 2009 (2009)

17. Colombo, R.M., Goatin, P., Rosini, M.D.: On the modelling and management of traffic. ESAIM: Mathematical Modelling and Numerical Analysis 45(05), 853-872 (2011)

18. Colombo, R.M., Rosini, M.D.: Pedestrian flows and non-classical shocks. Mathematical Methods in the Applied Sciences 28(13), 1553-1567 (2005)

19. Dafermos, C.M.: Polygonal approximations of solutions of the initial value problem for a conservation law. J. Math. Anal. Appl. 38, 33-41 (1972)

20. Dafermos, C.M.: Hyperbolic conservation laws in continuum physics, Grundlehren der Mathematischen Wissenschaften, vol. 325. Springer-Verlag, Berlin (2000)

21. De Lellis, C.: Notes on hyperbolic systems of conservation laws and transport equations. In: Handbook of differential equations: evolutionary equations. Vol. III, Handb. Differ. Equ., pp. 277-382. Elsevier/North-Holland, Amsterdam (2007)

22. Delle Monache, M., Goatin, P.: A front tracking method for a strongly coupled pde-ode system with moving density constraints in traffic flow. Discrete and Continuous Dynamical Systems - Series S 7(3), 435-447 (2014)

23. Delle Monache, M.L., Goatin, P.: Scalar conservation laws with moving constraints arising in traffic flow modeling: an existence result. Journal of Differential equations 257(11), 4015-4029 (2014)

24. Fan, S., Seibold, B.: Data-fitted first-order traffic models and their second-order generalizations: Comparison by trajectory and sensor data. Transportat. Res. Rec. 2391, 32-43 (2013)

25. Ferreira, R.E., Kondo, C.I.: Glimm method and wave-front tracking for the Aw-Rascle traffic flow model. Far East J. Math. Sci. (FJMS) 43(2), 203-223 (2010)

26. Garavello, M., Goatin, P.: The Aw-Rascle traffic model with locally constrained flow. J. Math. Anal. Appl. 378(2), 634-648 (2011)

27. Garavello, M., Piccoli, B.: Traffic flow on networks, volume 1 of AIMS Series on Applied Mathematics. American Institute of Mathematical Sciences (AIMS), Springfield, MO (2006)

28. Goatin, P., Colombo, R.M., Rosini, M.D., et al.: A macroscopic model for pedestrian flows in panic situations. GAKUTO International Series Mathematical Sciences and Applications 32, 255-272 (2010)

29. Godvik, M., Hanche-Olsen, H.: Existence of solutions for the Aw-Rascle traffic flow model with vacuum. Journal of Hyperbolic Differential Equations 05(01), 45-63 (2008)

30. Lattanzio, C., Maurizi, A., Piccoli, B.: Moving bottlenecks in car traffic flow: A pde-ode coupled model. SIAM Journal on Mathematical Analysis 43(1), 50-67 (2011)

31. Lax, P.D.: Hyperbolic Systems of Conservation Laws II. In: P. Sarnak, A. Majda (eds.) Selected Papers Volume I, pp. $233-262$. Springer New York (2005)

32. LeFloch, P.G.: Hyperbolic systems of conservation laws. Lectures in Mathematics ETH Zürich. Birkhäuser Verlag, Basel (2002)

33. Lighthill, M., Whitham, G.: On kinematic waves. II. A theory of traffic flow on long crowded roads. In: Royal Society of London. Series A, Mathematical and Physical Sciences, vol. 229, pp. 317-345 (1955)

34. Liu, T.P.: The Riemann problem for general systems of conservation laws. J. Differential Equations 18, 218-234 (1975)

35. Lu, Y.G.: Existence of global bounded weak solutions to nonsymmetric systems of Keyfitz-Kranzer type. Journal of Functional Analysis 261(10), $2797-2815$ (2011)

36. Mohan, R., Ramadurai, G.: State-of-the art of macroscopic traffic flow modelling. International Journal of Advances in Engineering Sciences and Applied Mathematics 5(2-3), 158-176 (2013)

37. Panov, E.: Generalized solutions of the Cauchy problem for a transport equation with discontinuous coefficients. In: Instability in models connected with fluid flows. II, Int. Math. Ser. (N. Y.), vol. 7, pp. 23-84. Springer, New York (2008)

38. Piccoli, B., Tosin, A.: Vehicular traffic: A review of continuum mathematical models. In: R.A. Meyers (ed.) Mathematics of Complexity and Dynamical Systems, pp. 1748-1770. Springer New York (2011)

39. Richards, P.I.: Shock waves on the highway. Operations Research 4(1), pp. $42-51$ (1956) 
40. Rosini, M.D.: The initial-boundary value problem and the constraint. Understanding Complex Systems pp. 63-91 (2013)

41. Rosini, M.D.: Macroscopic models for vehicular flows and crowd dynamics: theory and applications. Understanding Complex Systems. Springer, Heidelberg (2013)

42. Vol'pert, A.: The spaces BV and quasilinear equations. Math. USSR, Sb. 2, 225-267 (1968)

43. van Wageningen-Kessels, F., van Lint, H., Vuik, K., Hoogendoorn, S.: Genealogy of traffic flow models. EURO Journal on Transportation and Logistics pp. 1-29 (2014)

44. Zhang, H.: A non-equilibrium traffic model devoid of gas-like behavior. Transportation Research Part B: Methodological 36(3), 275-290 (2002) 